\title{
The role of the immune system and immune- derived signaling molecules in recovery from CNS injury
}

James Travis Walsh

Gibsonville, NC

Bachelor of Science in Chemistry (Biochemistry Concentration) and in Mathematics, University of North Carolina at Chapel Hill, 2005 
A Dissertation presented to the Graduate Faculty of the University of Virginia in Candidacy for the Degree of Doctor of Philosophy

Department of Neuroscience

University of Virginia

December 2014 


\section{Table of Contents}

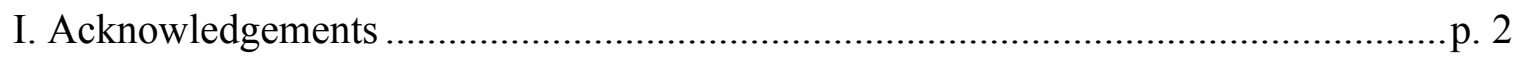

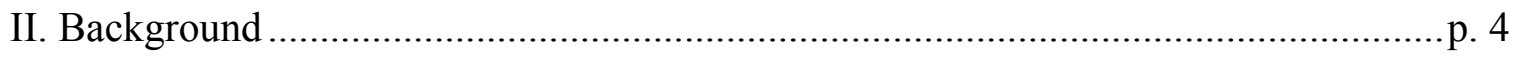

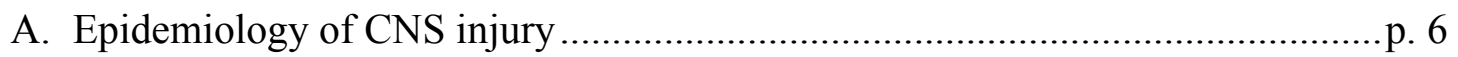

B. Pathophysiology of CNS injury .................................................................. 7

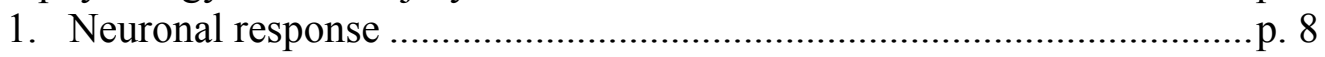

2. Astrocyte response........................................................................... 11

3. Oligodendrocyte/OPC response ………………............................ p. 14

C. Immune system response to CNS injury ………………............................... 17

1. Microglia and macrophages

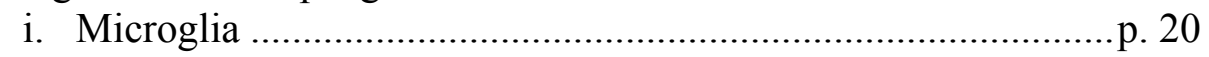

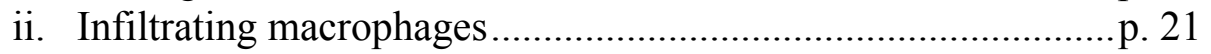

2. Neutrophils .............................................................................. 22

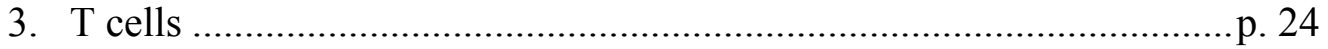

4. Other immune cells............................................................................. 33

D. Immune-derived signaling molecules in CNS injury ………….......................p. 33

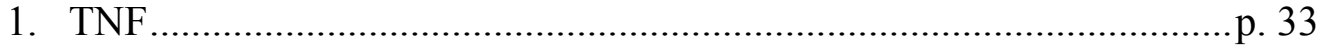

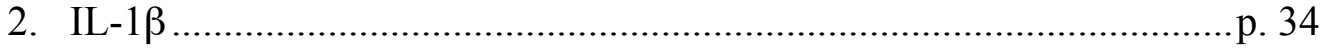

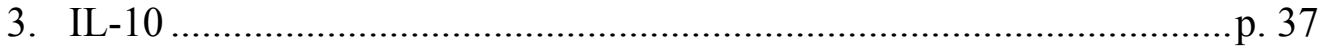

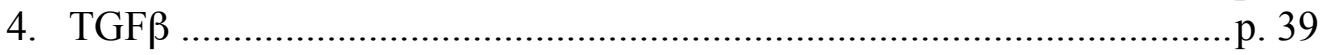

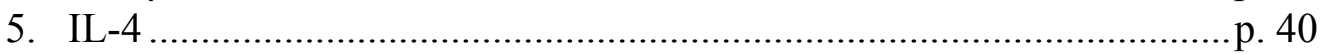

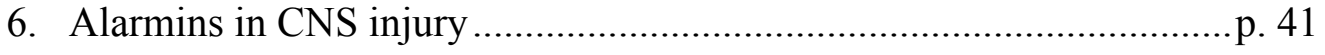

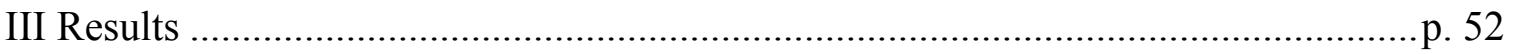

A. An antigen-independent immune response to CNS injury ................................. 52

B. The role of regulatory T cells in CNS injury ................................................... 69

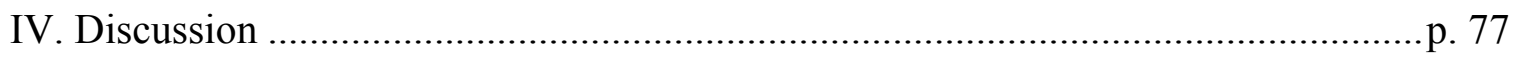

A. Antigen non-specific effects of T cells after CNS injury ................................... 80

B. IL-1 $\beta$ : protective secondary alarmin in the injured CNS …………………........ 81

C. Regulatory T cells in CNS injury .............................................................. 84

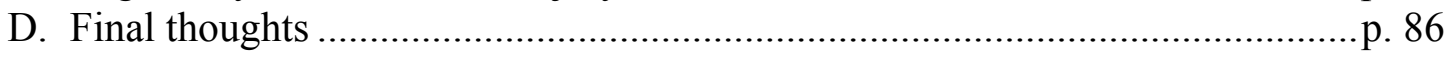

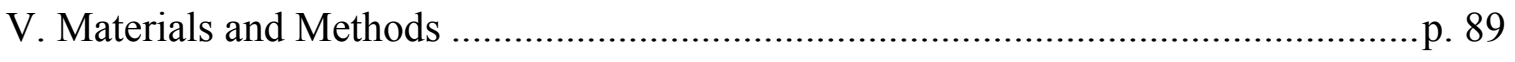

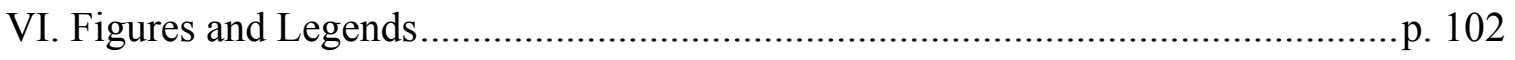

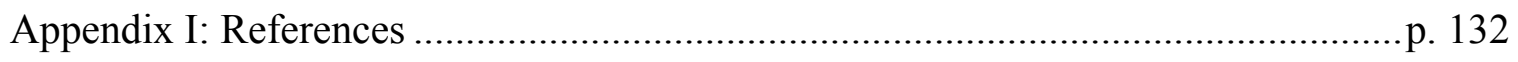

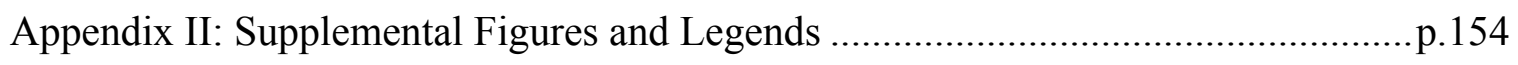




\section{Chapter I: Acknowledgements}

Coming in to grad school, I had no idea how many people it takes to do a thesis. You see the one name on the front, but without the assistance of several dozen people, nothing would have gone off the ground no matter how hard I tried. Though there's no way to thank everyone who has helped me in this endeavor, I would like to mention a few people specifically: I would like to thank the members of the Kipnis lab for the discussions I have had with them about my project. I have learned a tremendous amount from the incredibly intelligent people that I have interacted with. I would specifically like to mention two people that have been instrumental in the work presented here: Jinging Zheng for the work she put in on the $\mathrm{T}_{\text {reg }}$ project and, most importantly, Igor Smirnov, without whose invaluable assistance with all my projects I would not have been able to achieve what I did in my time here.

I would like to acknowledge the hard work that Jony has put in teaching me both the art and the politics of science. Though he was sometimes a hard taskmaster and his ideas sometimes seemed off the wall, he was more often than not right, and his tireless pursuit of the underpinnings of the systems that we studied motivated me to become the scientist I am today. I would like to thank him for staying positive through the paper rejections and times where experiments hit roadblocks that seemed like they would never clear up.

I would like to thank my family for all their support: to my parents for always encouraging me and believing in me, and for their example of how to work hard and to do things the right way. 
And though there is no way to properly express my gratitude, I want to thank my incredibly understanding wife, Claudia. She has encouraged me even though it meant late nights in the lab doing experiments, weekends where I couldn't leave town because my mice needed care, and for putting up with me when where I'd come home frustrated with the rejections that inevitably come along with being in a peer-reviewed field. Without her, grad school might well have been overwhelmed me, and I am incredibly lucky that she has been with me during this time. 


\section{Chapter II: Background}

The central nervous system (CNS), consisting of the brain and spinal cord, is the body's control system, relaying instructions that control every facet of life, from conscious decisions and activities to unconscious autonomic responses that maintain homeostasis day to day. The CNS develops through a complex set of instructions starting in embryogenesis, and continuing through the early postnatal period. However, neurogenesis ends in the early post-natal period except for in a few specialized regions, and the terminally differentiated neurons that inhabit the adult CNS are post-mitotic, making the majority of these neurons that are present in adulthood irreplaceable (Deng et al., 2010). Due to its importance and its inability to regenerate, the CNS is protected from injuries by the skull and spinal column, bony structures surrounding this vital and sensitive organ. However, when injuries do happen to the CNS, the result is often permanent disability, with little prospects for full recovery or even viable therapeutic prospects, highlighting the need for more research for this devastating pathology (Plemel et al., 2014).

A promising line of investigation on how to ameliorate the impact of disability from CNS injury is to prevent the continued degeneration of the neurons that occurs within the first weeks after trauma, termed 'secondary degeneration' (Yoles and Schwartz, 1998). Evidence has accumulated over the last 15 years that has made it clear that $\mathrm{CD}^{+} \mathrm{T}$ cells can prevent this secondary degeneration (Frenkel et al., 2005; Hauben et al., 2000; Kipnis et al., 2002b; Moalem et al., 1999), yet CD4-based therapy has not been seriously pursued because of the dangers that have presented themselves with $\mathrm{CD} 4^{+}$ 
lymphocyte activation in the CNS. In order to understand how to harness this $\mathrm{CD}^{+}$ mediated neuroprotection, the mechanisms that underlie the protection and destruction they mediate need to be better understood. Unfortunately, previous work that has gone into elucidating the mechanisms leading to lymphocyte-mediated neuroprotection has not provided a sufficient depth of understanding. For example, the role of $\mathrm{T}$ cell subsets and their primary cytokines in neuronal survival has not been fully addressed. Similarly, while the adaptive immune responses are often initiated in the draining lymph nodes, it was unclear whether this was a relevant concept in CNS injury, and if so, which lymph nodes would harbor the immune response to CNS injury. The major black box in the response to CNS injury, however, is the mechanism by which the immune response to injury is initiated. While antigen-specific $\mathrm{CD}^{+} \mathrm{T}$ cells are especially potent at promoting neuroprotection in wild type mice, whether this antigen recognition by $\mathrm{T}$ cells in the context of MHCII is necessary to induce the immune response to injury, or whether there are alternate signals that lead to the initiation of the response, is not known.

Below, I will summarize some of the seminal works that have shaped our view of the cellular and molecular response to CNS injury. I will then present my own about how the adaptive immune response is initiated and how these cells affect CNS injury once activated. Finally, I will discuss how these findings fit in to the body of work that has been collected thus far.

\section{Epidemiology of CNS injury}


CNS injuries can be divided into two classes: spinal cord injury and traumatic brain injury. While the medical challenges of treating these diseases vary slightly, they share many common traits. Because the bony structures that protect the CNS need to be damaged for there to be injury to the CNS, these injuries tend to occur when there are large forces acting on the CNS. Therefore, the populations that are affected by these injuries are disproportionately the young, who engage in reckless behavior, and the old, whose bones tend to be more brittle and who are prone to falls (Sekhon and Fehlings, 2001). Injuries to the brain and spinal cord lead to long-lasting sequelae, which often are never completely resolved. For example, in one study of patients that were admitted with mild traumatic brain injury, only $19 \%$ of patients were able to return to work at full capacity in 6-9 months (Ruffolo et al., 1999). Similarly, that number is $19 \%$ who were able to return to work at one year post-injury and $40 \%$ of patients that are never able to return to work after spinal cord injury (Center, 2013).

Because of the propensity to affect the young (Langlois et al., 2005) and the chronic nature of these diseases, they represent a burden of disease that is vastly disproportionate to their incidence. While spinal cord injuries have an incidence of 1220,000/per year (Bernhard et al., 2005), they cost $\$ 7.7$ billion in direct medical costs (DeVivo, 1997). Similarly, traumatic brain injury (TBI) has an incidence of 1.6 million per year, and costs $\$ 9.2$ billion in direct medical costs (Rutland-Brown et al., 2006). However, the real burden of these diseases lies in their loss of productivity. Because of this propensity to affect young people and the life-long disability that they cause (Ruffolo et al., 1999), they represent a major cause of loss of productivity, despite only affecting a small percentage of the population (Center, 2013). Therefore, the indirect cost of these 
injuries also adds a significant burden of disease to our society, adding an additional \$2.6 billion for spinal cord injury and a staggering $\$ 51.2$ billion to the cost of TBI (RutlandBrown et al., 2006).

\section{Pathophysiology of CNS Injury}

The CNS is made up of several different cell types that provide for the action of the CNS. Principally, there are the neurons, which are specialized for conducting and integrating the electrical signals that represent the primary function of the CNS. The speed of this electrical conduction is increased by the wrapping of neuronal axons by the plasma membrane of oligodendrocytes, providing for the faster saltatory conduction of electrical signals. Astrocytes are glial cells that form a structural support to the CNS, help fine-tune electrical signaling, and can act as one of the first lines of defense against CNS pathologies. Oligodendrocyte precursor cells (OPCs) are a newly-appreciated cell type that can give rise to oligodendrocytes, as their name implies, but whose other functions are just now beginning to be understood. Finally, there are the microglia, yolk-sac derived cells (Kierdorf et al., 2013; Schulz et al., 2012) that have many similarities to tissue-resident macrophages found throughout the rest of the body (David and Kroner, 2011). While the role of microglia in infection and injury has been well studied (Rivest, 2009), their role in normal physiology is just beginning to be understood (Salter and Beggs, 2014). Each of these cell types, along with the infiltrating immune system, has its own unique response to CNS injury and contributes to the orchestrated response that has evolved as a consequence of CNS injuries.

\section{Neurons}


As the cells that are specialized to conduct the signals that are the primary role of the CNS, the reaction of neurons is one of the most important and the best studied responses to CNS injury. The response of neurons occurs at two principal locations: at the axon, in the instance of axonal damage, and at the cell body in the instance of both axonal or cell soma damage. The response at each of these locations has its unique role in the injury response.

Neuronal response to axonal damage at the site of injury

Especially important in the majority of CNS injury situations are the thin, fragile axons, which are especially sensitive to damage in traumatic injury (Meythaler et al., 2001). Initially after axonal injury, there is a loss of membrane integrity that allows extracellular ions to enter the intracellular space, and which is the first challenge faced by the injured neuron. While this influx of ions, and especially extracellular calcium, is vital to the recovery from axonal damage (Ghosh-Roy et al., 2010), the plasma membrane of the axon needs to be quickly resealed in order to promote survival and proper function. The influx of calcium from the extracellular space, and resulting release of calcium from intracellular stores, promotes sealing of the plasma membrane by collapse of the cytoskeleton at the damaged axonal end (Spira et al., 1993), followed by the formation of a membranous plug to help restore ionic homeostasis (Eddleman et al., 1998). Once the membrane has been resealed, the damaged neuron will begin to perform cytoskeletal rearrangements to produce a retraction bulb and/or growth cone (Erez and Spira, 2008). At this point, the neuron has survived from the initial membrane damage, but several 
factors can still drive it down the apoptotic pathway depending on the responses that occur at the cell body.

Neuronal cell body response to injury

Once an axon or cell body of a neuron is damaged and re-sealed, it has a simple choice to make: try to repair the damaged connections, or undergo apoptosis and be cleared by phagocytes in the tissue in a non-inflammatory manner. However, the process that goes into this decision is complicated. Neurons can die by both apoptotic and necrotic mechanisms after injury. Primary neuronal death, due to membrane damage that leads to an altered intracellular ionic imbalance as described above, is primarily a necrotic event due to the initial loss of membrane integrity that is compounded by the activation of clapain proteases that, despite their importance in axonal remodeling and regrowth, can also induce necrosis if unchecked (Farkas et al., 2006).

After this wave of primary degeneration, a host of factors lead to continuous degeneration beyond what would have been expected from the cells that were damaged by the initial insult (Yoles and Schwartz, 1998). Among the mechanisms for this secondary cell death is ionic imbalance both of calcium (the constitutive signaling of which will induce cytochrome c release from the mitochondria and apoptosis (Smaili et al., 2009)), excitotoxic cell death due to the release of glutamate (Lipton and Nicotera, 1998) and other signaling molecules released from neurons that have undergone necrosis (Franke and Illes, 2006), a loss of trophic support due to the loss of axonal innervation of the target tissue (Rich, 1992), and reactive oxygen species production both from the innate immune response to injury (Bao et al., 2009) and from the mitochondrial dysfunction secondary to ionic imbalances (Cheng et al., 2012). 
Among the best studied of these mechanisms of secondary degeneration after injury is the loss of trophic support that is necessary for the survival of most CNS neurons (Rich, 1992). This phenomenon was first discovered in dorsal root ganglion cultures, where it was found that loss of NGF would cause a robust apoptosis of neurons from the ganglion, while addition of exogenous NGF would promote exaggerated axonal outgrowth (Levi-Montalcini, 1987). Subsequently, several more members of this family have been discovered, including NT3, NT4, and BDNF (Vicario-Abejón et al., 2002). These molecules signal by binding their ligands to the Trk receptors in the axon (or paradoxically in a way that causes neuronal apoptosis through the $\mathrm{p} 75^{\mathrm{NTR}}$ receptor at the cell body (Deppmann et al., 2008)), which are then transported in a retrograde fashion to the cell body, where they are able to complete their signaling (Reichardt, 2006). In injured neurons, axonal transport is one of the first processes that is disrupted (Coleman, 2005), and the loss of this signaling subsequent to the loss of axonal transport can lead to apoptosis.

Reactive oxygen species (ROS) are byproducts of mitochondrial respiration that occurs in every nucleated cell (Benz and Yau, 2008). These molecules are characterized by an unpaired electron that can cause a chain reaction, breaking and re-forming bonds of many different macromolecules that would otherwise be stable under physiological circumstances. Normally, these radicals are well controlled within the mitochondrial respiratory chain, and there are cellular mechanisms that can repair the damage that occurs with small amounts of oxidative damage. However, excessive ROS production that can occur either in a regulated fashion by the immune system (Dupré-Crochet et al., 
2013) or due to abnormal mitochondrial respiration (Cheng et al., 2012) can lead to irreparable DNA damage, a signal for cellular apoptosis.

Finally, excitotoxicity is a process that can lead to both necrotic and apoptotic death of neurons. Glutamate released from cells that have previously died will induce constitutive signaling in neurons, increasing intracellular calcium via influx from the extraellular space. This in turn releases intracellular calcium stores, which can induce apoptosis through mechanisms discussed earlier. It also induces activation of calpains and cathepsins, stimulating proteolysis and breakdown of cell membrane integrity in a process termed necroptosis, due to the fact that it is a regulated process like apoptosis, but releases inflammatory intracellular components like in necrosis (Dunai et al., 2011).

\section{Astrocytes}

Astrocytes are supporting cells of the CNS that play multi-functional roles in the physiological functioning of the CNS. In addition to the well-established role of astrocytes as structural support for the CNS, the last 20 years have seen an increased appreciation for their role in other physiological functions. They are able to control the blood flow to different areas of the brain through their production of prostaglandins, nitric oxide, adenosine, and glutamate, a role they are well-adapted for by their intimae contact with blood vessels (Filosa and Iddings, 2013). They also have recently been shown to be involved in neuronal signaling, both morphologically, with processes within nanometers of the synaptic cleft (Lehre and Rusakov, 2002), and chemically, by their role in glutamatergic signaling (though there is still debate as to whether this action is simply through glutamate uptake, or if they also have the ability to actively release the 
neurotransmitters they have previously taken up (Parri et al., 2001)). This interaction of astrocytes with the neuronal synapse is dubbed the tripartite synapse, indicating that not only are the pre- and post-synaptic membranes important, but so is the astrocytic involvement (Araque et al., 1999). In addition to their role in neuronal signaling and structural support, astrocytes also play important roles in glutamate uptake from the extracellular space (Bernardinelli et al., 2004), synaptogenesis (Christopherson et al., 2005), neuronal metabolism (Pellerin et al., 1998), and control of water homeostasis through aquaporin 4 (Nagelhus and Ottersen, 2013).

As with the adaptive immune response in CNS injury (which we will delve into more later), both neuroprotective and neurodestructive roles have been ascribed to astrocytes (Seifert et al., 2006). The astrocytes that respond to injury or infection have been termed the "reactive astrocyte," which are characterized by a change to a hypertrophic morphology and upregulation of the prototypical astrocyte marker GFAP as well as two other intermediate filaments: nestin and vimentin (Zamanian et al., 2012). In a seminal work by the Sofroniew lab (Bush et al., 1999), many of the roles of astrocytes were elucidated that have been explored further in studies since then; ablation of injuryreactive astrocytes leads to an increase in blood brain barrier (BBB) permeability, an increase in immune cell infiltration into the injury site, and an increase in both neuronal degeneration and axonal regrowth through the site of injury (Bush et al., 1999). Consistent with the protective role of astrocytes acutely after injury, conditional ablation of STAT3, a signaling molecule that is vital for astrocyte activation, leads to an increase 
in lesion size and a decrease in functional recovery from injury (Herrmann et al., 2008; Okada et al., 2006).

Even when trying to isolate the effect of individual functions of astrocytes, pigeonholing these responses as simply beneficial or detrimental is not an easy task. For example, they are vital players in the maintenance and re-establishment of the BBB after injury through their production of basement membrane proteins, known as the glia limitans (Kawano et al., 2012). They produce extracellular matrix components such as tenascins, neurocans, phosphocans, and thrombospondins, to name a few (BonnehBarkay and Wiley, 2009; Christopherson et al., 2005), as well as promote tight junctions formation on endothelial cells that further inhibits the pathological movement of cells and macromolecules from circulation into the CNS (Willis et al., 2004). After an injury, astrocytes produce many of the extracellular components that make up the glial scar (Fitch and Silver, 2008). While this walls off the site of injury and prevents the diffusion of the detrimental components that are released from systemic circulation and dying cells towards the injured tissue (Bush et al., 1999), it also adds a layer of inhibitory molecules, such as chondroitin sulfate proteoglycans (CSPGs) and tenascins, which provide a barrier to axonal regrowth. These molecules cause the collapse of the growth cone in neurites and stand as one of the major barriers to axonal regeneration in the CNS after injury (Watkins and Barres, 2002).

With the importance of astrocytes in physiological functioning of the CNS, it is probably not surprising that astrocytes are not only important at the lesion epicenter through their production of the glial scar components, but also play a role distantly where 
there is degeneration of cell bodies and axons after CNS injury. As with reactive astrocytes at the injury site, these cells will have enhanced STAT3 signaling and a reactive phenotype, and promote optimal survival and recovery after injury (Tyzack et al., 2014). They can engulf both degenerating axons (Ziegenfuss et al., 2012) and cell bodies (Chang et al., 2000), a process that prevents an inflammatory secondary necrosis (Lööv et al., 2012), confirming that, despite the attention that has been paid to the reactive astrocyte in forming the glial scar, these cells also contribute to the recovery in many other ways.

In line with this idea of this multifaceted astrocyte response to CNS injury, they can buffer extracellular ions and neurotransmitters through transporters such as excitatory amino acid transporters (EAATs) and thus reduce excitotoxic damage to bystander cells (Beschorner et al., 2007). However, in a maladaptive response, this buffering capacity becomes diminished after injury (van Landeghem et al., 2006), consistent with the idea that these cells sacrifice their physiological roles to become more specialized "reactive" cells after injury. Finally, astrocytes also produce several chemokines and cytokines that initiate the immune response to injury and direct the immune response, which we will discuss in depth later.

\section{Oligodendrocytes/OPCs}

In line with their role as structural elements of the CNS whose primary function is to insulate the axons to provide faster conduction, oligodendrocytes are considered to be passive responders to CNS injury. While much work has focused on their death and 
survival in CNS injury, very few studies have undertaken the task of determining if and how they respond to injury in an adaptive manner.

Oligodendrocytes have been of great interest in the CNS injury field, however, because the molecules they produce basally can influence the secondary recovery after injury and because their presence is vital for the physiological function of neurons (Sherman and Brophy, 2005). It has been proposed that oligodendrocyte loss can directly cause loss of neuronal viability, due to several mechanisms. Loss of oligodendrocytes promotes re-arrangement of sodium channels along the entire length of the axons, causing an increased energy demand for neurons (Moll et al., 1991) and subsequently an increase in intracellular calcium (Nikolaeva et al., 2005), one of the major triggers of apoptotic cell death. Further work has suggested that oligodendrocytes can promote neuronal health independently of their ability to myelinate. Mice deficient in the oligodendrocyte proteins PLP (Griffiths et al., 1998) and CNP (Lappe-Siefke et al., 2003), as well as loss of oligodendrocyte peroxisomes (Kassmann et al., 2007), display progressive neurodegenerative changes including loss of neurons. While the exact mechanism of these mutations has yet to be elucidated, it seems that they can be segregated from the deficit in myelination (Kassmann et al., 2007), suggesting that oligodendrocytes are also providing additional trophic support for the axons they wrap.

Finally, oligodendrocyte corpses cause a unique problem to the remodeling CNS. Because oligodendrocytes produce so much membrane in the form of their myelin sheath, they can overwhelm the phagocytic ability of microglia and their capacity to degrade the debris. As with atherosclerotic plaques, clearance of the debris is incomplete even years 
after CNS injury (Becerra et al., 1995; Buss et al., 2004), partially due to inefficiencies in phagocytosis in the CNS (Gitik et al., 2011) (but surprisingly, this clearance is efficient in the PNS, which has greater potential for regrowth (Gaudet et al., 2011), possibly due to the robust immune cell infiltration after PNS injury (Hirschberg and Schwartz, 1995)). The unprocessed debris prevents axonal regrowth after injury, as it includes endogenous axonal inhibitory extracellular molecules produced by the oligodendrocyte, including CSPGs, Nogo, MAG, and OMgp (Chen et al., 2000; McKerracher et al., 1994; Wang et al., 2002; Zuo et al., 2002)

As with oligodendrocytes, there is limited evidence about the role of $\mathrm{NG2}^{+}$ oligodendrocyte precursor cells (OPCs) after CNS injury. Unlike oligodendrocytes, OPCs are thought to actively respond to CNS injuries and to promote the proper recovery from injury (Wu et al., 2012). As with microglia, OPCs display chemotaxis towards the lesion center, albeit with delayed kinetics, probably due to the fact that they are responding to signals derived from the reactive microglia instead of signals directly from the injured tissue, though the exact identity of this OPC chemoattractive signal is unknown (Rhodes et al., 2006). At the injury site, they respond first as undifferentiated OPCs (Glezer et al., 2006), then as differentiated oligodendrocytes that remyelinate regrowing axons (Wu et al., 2012). Because of their ability to differentiate into oligodendrocytes, there is a paucity of good studies on the role of the undifferentiated OPCs in vivo; however there are some hints as to the activity of OPCs. Several groups have injected OPCs into injured spinal cords to find that they promote functional recovery from injury (Sharp et al., 2010; Sun et al., 2013), though the contribution of remyelination and neuroprotection is still a 
matter of debate. Some initial evidence shows that OPCs can promote neuroprotection independently of their ability to myelinate, however; injection of OPCs into the retina, which is devoid of myelin, promotes neuronal survival through an interaction with inflammatory cells (Bull et al., 2009), suggesting that even as undifferentiated cells these OPCs were playing a neuroprotective role. Further evidence demonstrates that OPCs can also act as a substrate for dystrophic axons (Busch et al., 2010), though, again, the importance of this affinity for regrowing axons to NG2 glia in vivo is still very much a matter of debate: while $\mathrm{NG}^{+}$glia prevent regrowth from cerebellar explants (Chen et al., 2002), they also promote the growth of isolated neurons (Busch et al., 2010), suggesting that they produce axonal-supporting molecules in addition to axonal-inhibitory CSPGs such as NG2.

\section{The immune response to CNS injury}

Because of the highly adaptive nature of the immune system, it is an ideal place to intervene to promote recovery after CNS injury: it is readily accessible through the peripheral circulation, it hones in on the injured tissue (Popovich et al., 1997a), and it affects the outcome from injury. However, the complexity with which the immune system responds to injury and the potential for it to cause damage on its own (Miller et al., 2007) means that the responses to injury must be well understood before they could potentially be harnessed clinically. Described below are some of the better-understood players in the immune response to injury, both cellular and molecular.

\section{Microglia/macrophages}


As compared to macrophages that infiltrate the CNS injury site, which are derived from bone-marrow derived monocytes, microglia originate from the embryonic yolk sac (Kierdorf et al., 2013). After seeding of the embryonic CNS, these long-lived cells selfpropagate throughout adulthood, and remain the only immune cells in the CNS parenchyma under physiological conditions (Ransohoff and Brown, 2012). Despite the fact that they are considered quiescent under these circumstances, it is beginning to become understood how they play a vital role in homeostasis of the CNS. They are responsible in synaptic pruning that is necessary for proper CNS development (Bialas and Stevens, 2013; Salter and Beggs, 2014; Schafer et al., 2012) and play an important role in learning, memory, and proper neural functioning in health (Derecki et al., 2010) and disease (Derecki et al., 2012). However, the response of microglia to damage to the CNS through infection or injury still remains the best-studied role of these versatile cells.

Because the distinct functional roles microglia and macrophages play in CNS injury were not appreciated until recently, most of the data on their response to injury does not discriminate based on which cell type is mediating the response. Therefore, early works lump the activity of these cells together. Therefore, we will begin the discussion of the myeloid cell role in injury with early results whose function has not been assigned to a particular cell type before delving into the different roles that have been ascribed to microglia or macrophages independently. Indeed, the initial lumping of these macrophages derived from infiltrating monocytes and the resident microglia is understandable, as both of these cells are specialized to phagocytose extracellular debris and to produce cytokines that affect their milieu, and upon activation there is no marker 
that distinguishes them. Rather, they can only be reliably separated by fate mapping or by their susceptibility to irradiation.

As with targeting many of the cell types or molecules involved in CNS injuries, interpreting the results of targeting of macrophages/microglia after injury is not a simple proposition, but rather the outcome depends on the timing of the intervention and the mechanism of the myeloid manipulation. Initial experiments targeting this population non-specifically lead to the hypothesis that the myeloid response to CNS injury was a detrimental response. Minocycline is a synthetic tetracycline antibiotic that has been shown to reduce myeloid pro-inflammatory cytokine and nitric oxide synthase (iNOS) production (Tikka et al., 2001). Global inhibition of the myeloid response with minocycline has been assessed in several different models, and has generally yielded beneficial results (reviewed in (Yong et al., 2004)). Similarly, treatment with clodronate liposomes, which induce apoptosis in phagocytic cells, also led to an increase in functional recovery from spinal cord injury (Popovich et al., 1999), which was supported by in vitro experiments showing axonal retraction in response to activated myeloid cells (Horn et al., 2008). However, neither of these treatments are without off-target effects: minocycline has a direct effect promoting neuronal and oligodendroglial survival, complicating the myeloid effects of this drug (Teng et al., 2004) and clodronate liposome treatment lacks specificity to myeloid cells and will affect all phagocytic cells, not just in the brain but globally. Unfortunately, these studies are probably oversimplifications of the myeloid cell response to injury, and while the debate still rages today about what effects these cells are having, it is important to recognize that the microglial response 
differs from the monocyte-derived macrophage response, and that the effects of these populations need to be studied separately.

\section{Differential effects of microglia and infiltrating macrophages : microglia}

Within minutes of an injury to the CNS, microglia change their morphology from a ramified phenotype (often considered "resting") to an amoeboid "reactive" phenotype and migrate to the site of injury (Davalos et al., 2005). Upon this activation, they upregulate markers such as Iba1, CD11b, CD45, and MHCII, and begin to acquire functional activity such as phagocytosis and reactive oxygen species production, as well as production of cytokines and chemokines (David and Kroner, 2011). Recent work by Greenhalgh and David using a model that expresses EGFP under the LysM promoter (which is found on circulating monocytes but is not on resident immune cells) showed that early after injury, microglia were the main phagocytes in the injured spinal cord and efficiently processed the material they ate. In contrast, infiltrating macrophages were the predominant phagocyte after several days, but were prone to apoptosis and contained debris even 6 weeks after injury (Greenhalgh and David, 2014), suggesting that microglia were playing an adaptive role in the recovery to CNS injury. Similarly, experiments done in slice cultures suggested that M2-skewed microglia could be protective, whereas bone marrow derived macrophages, a proxy for the infiltrating inflammatory macrophages, were destructive even if pre-conditioned towards an M2 phenotype (Girard et al., 2013). However, not all reports have suggested that microglia are playing beneficial roles. Recently, microglia in inflammatory conditions have been shown to assume a dramatic phenotype in neuronal death by displaying the ability to eat living neurons in a process 
coined phagoptosis (Fricker et al., 2012), though the relative importance of phagoptosis amidst the massive cell death seen in CNS trauma is still unknown.

\section{Differential effects of microglia and infiltrating macrophages: infiltrating macrophages}

In contrast to microglia that are constitutively present in the CNS tissue, bloodborne monocytes begin to infiltrate into the injured CNS days after injury (Shechter et al., 2009). As with microglia, there is a controversy in the literature about whether these cells are protective or destructive in traumatic injury. Several groups have showed that infiltrating macrophages promote axonal death in vivo (Evans et al., 2014; McPhail et al., 2004), and blockade of this immune cell infiltrate into the CNS using CCR2 $2^{-/-}$mice led to enhanced functional recovery after injury (Semple et al., 2010a). In contrast to the destructive roles that are highlighted above, recent works, especially that from the Schwartz lab, advocate for the protective role of tissue-infiltrating monocytes (London et al., 2013). Early work showed that treatment with macrophages activated by peripheral tissue (where there is a robust immune response and abundant regeneration) lead to an axonal outgrowth in the optic nerve, where regrowth is not normally seen (LazarovSpiegler et al., 1996). In a spinal cord injury model, this regrowth induced by activated macrophages could lead to an increase in the functional recovery from a severe spinal cord injury (Rapalino et al., 1998). Also in a severe spinal cord injury model, they showed that infiltrating macrophages that upregulated CX3CR1 did not inhabit the lesion epicenter, but stayed on the periphery and had an anti-inflammatory phenotype characterized by production of IL-10 and arginase 1 (Shechter et al., 2009). Depletion of 
these macrophages using a DTx-mediated depletion in bone marrow chimeras or systemic depletion of all circulating monocytes using CCR2 antibodies led to decreased functional recovery from injury (Shechter et al., 2009). Further work from this group showed that infiltrating monocytes are not a homogeneous group of cells, but rather represent two different lineages: so-called "inflammatory" macrophages that are $\mathrm{CX} 3 \mathrm{CR} 1^{\text {low }}$ and $\mathrm{CCR} 2^{\text {hi }}$, and resident macrophages that are $\mathrm{CX} 3 \mathrm{CR} 1^{\text {hi }}$ and CCR2 $2^{\text {low }}$. They further show that selectively blocking the resident monocyte infiltration into the injury site was sufficient to prevent optimal functional recovery after spinal cord injury (Shechter et al., 2013).

While it is now widely accepted that alternatively activated macrophages, as evidenced by expression of arginase 1 and/or IL-10, are protective after CNS injury (Fenn et al., 2014; Kigerl et al., 2009; Shechter et al., 2009) and are derived, at least partially, from infiltrating monocytes (Shechter et al., 2009), what initiates and sustains this phenotype is still not well understood. While there is ample evidence for molecules that can cause macrophage skew in vitro, it is still unknown whether any of these molecular players are having a role in the macrophage phenotype acquired after the injury.

\section{Neutrophils}

Neutrophils are short-lived innate immune cells that are specialized to phagocytose debris and activate the respiratory burst for pathogen clearance (Mantovani et al., 2011). These cells are among first infiltrating responders to injury, appearing in the lesion within hours of CNS injury and peaking around 24 hours (Nguyen et al., 2008). 
The neutrophil response to CNS injury is generally detrimental after injury. Prevention of neutrophil migration into injured CNS tissue with $\mathrm{CXCR} 2^{-/-}$mice reduces neuronal loss from injury, although it had no effect on functional recovery (Semple et al., 2010b), while pharmacological inhibition of CXCR2 similarly decreased lesion size, but additionally promoted increased functional recovery from injury (Gorio et al., 2007). Using an alternative method of preventing neutrophil influx into the injured tissue, $\mathrm{C} 5^{-1-}$ mice were shown to have a deficit in neutrophil infiltration into the site of cryo-injury that correlated with a decrease in lesion size (Sewell et al., 2004). Also, depletion of neutrophils specifically, using Ly6G antibody-mediated depletion, led to a better functional outcome from injury (Sansing et al., 2011). In contrast (and probably better highlighting the importance of infiltrating monocytes in recovery from CNS injury), injection of the GR-1 antibody, which recognizes both Ly6C, found on monocytes, and Ly6G, found on neutrophils, lead to the opposite effect: greater white matter degeneration and a worsened functional outcome (Stirling et al., 2009). Despite the overwhelming evidence that the acute neutrophil response to CNS injury is maladaptive, there is more work to be done to determine if these cells also can play protective roles after injury. Indeed, work in optic nerve injury by the Benowitz lab has shown that neutrophils can mediate regrowth of retinal ganglion cell axons (Kurimoto et al., 2013), suggesting that these cells might also be playing a role in recovery from injury; however, whether this regrowth-promoting response to neutrophils is relevant in a clinical setting is still unknown.

\section{T cells}


Early dogma in the CNS injury field was that an adaptive immune response to CNS injury was always detrimental (Jones et al., 2002). Strikingly, however, the secondary degeneration is more extensive in animals lacking an adaptive immune system than in their wild-type counterparts, suggesting a previously unknown neuroprotective role for the adaptive immune cells (Moalem et al., 1999; Serpe et al., 1999; Yoles et al., 2001). Restoration of the immune system, and particularly of the T cell compartment, in immune-deficient mice (SCID or nude) restores their normal response to CNS injury (Kipnis et al., 2002a; Serpe et al., 2003) further suggesting that an endogenous immune response to CNS injury is neuroprotective. Importantly, it was discovered that not all T cells can mediate this neuroprotective effect, but that the $\mathrm{T}$ cells need to be specific to brain-restricted antigens (Moalem et al., 1999), which probably governs their migration to, and accumulation in, the injured CNS (Archambault et al., 2005; Ling et al., 2006). Thus, transfer of autoreactive $\mathrm{T}$ cells directed against the CNS antigen, myelin basic protein (MBP), reduced the secondary degeneration after nerve injury in rats, and this neuroprotection could be provided through both active immunization (via immunization with spinal cord homogenates or purified myelin proteins and adjuvant), or passive immunizations (through the transfer of pre-activated CNS-specific T cells) (Byram et al., 2004; Hauben et al., 2000; Moalem et al., 1999).

There are several functions of these $\mathrm{T}$ cells, both canonical (cytokine producing) and non-canonical, that can contribute to their neuroprotective phenotype after CNS injury. The non-canonical effects of $\mathrm{T}$ cells that contribute to the recovery from injury often rely on interactions with neural cells or the injured neurons themselves. For 
instance, $\mathrm{T}$ cells can protect neurons directly through production of neurotrophic factors, such as brain-derive neurotrophic factor (BDNF), when they become activated (Kerschensteiner et al., 1999; Moalem et al., 1999). This immune-derived BDNF plays a functional role in the injured CNS, and mice that lack BDNF in their myeloid and $\mathrm{T}$ cell populations exhibit deficits in functional outcomes after inflammatory injury (Linker et al., 2010). Additionally, glial cells are involved in the $\mathrm{T}$ cell protective response, as they can signal to astrocytes to up-regulate the production of protective thiol compounds and increase their buffering of glutamate (Garg et al., 2008; Garg et al., 2009). In addition to these cytokine-independent mechanisms for $\mathrm{T}$ cell mediated neuroprotection, several recent works have begun to unravel importance of cytokine-dependent interactions between $\mathrm{T}$ cells and myeloid cells in the outcome of CNS injury. Monocytes that are recruited to the site of injury in a CCR2-dependent manner take on an alternatively activated phenotype as evidence by expression of IL-10, while the resident microglia do not display this alternatively activated phenotype (Shechter et al., 2009). Additionally, the peak of alternative activation of myeloid cells after injury coincides with the peak of $\mathrm{T}$ cell infiltration into the CNS, suggesting a beneficial $\mathrm{T}$ cell effect on myeloid phenotype (Miron et al., 2013; Popovich et al., 1997b). These alternatively activated macrophages are considered tissue building/healing, and can produce trophic molecules, such as IGF-1 and TGF $\beta$, that are important in growth and development and promote recovery in injured CNS tissue (Kigerl et al., 2009). Importantly, one of the best-studied routes to produce alternatively activated macrophages is through signaling by IL-4, a prototypical Th2 cytokine (Anthony et al., 2006). T cells, then, can infiltrate into the 
tissue and produce Th2 cytokines, contributing to the protective alternatively activated macrophage skew.

Regulatory T cells - regulators of the immune response to injury

As described above, there is ample evidence that $\mathrm{T}$ cells can play a protective role after injury, although an even more substantial literature exists on the pathological effects of autoimmune T cells in the CNS, such as in multiple sclerosis and neuromeylitis optica (Bettelli et al., 2006a; Paterson, 1960). Therefore, if the same T cells could be protective and destructive - what regulates and dictates their function? A key player in controlling autoimmune responses that might hold the answer to this paradox was uncovered some 40 years ago with the discovery that a population of lymphocytes could control adaptive immune responses (Gershon et al., 1972).

Some twenty years after their initial discovery, the discovery of the molecular identity of these $\mathrm{CD} 25^{+}$regulatory $\mathrm{T}\left(\mathrm{T}_{\text {reg }}\right)$ cells sparked a revolution in our understanding of how the immune system is controlled (Sakaguchi et al., 1995). These regulatory $\mathrm{T}$ cells are marked by expression of the transcription factor Foxp3 (Fontenot et al., 2003; Hori et al., 2003; Khattri et al., 2003) and have been proposed as the key player in controlling autoimmune responses by the adaptive immune system. Thus, this subset of $\mathrm{T}$ cells acts not to increase the activity of the immune system to salient stimuli, but instead to act as an endogenous brake to ensure that adaptive immune responses are correctly measured in response to their stimuli (Sakaguchi et al., 1995). Therefore, it stands to reason that $\mathrm{T}_{\text {reg }}$ cells would be detrimental to the autoreactive- $\mathrm{T}$ cell response so 
necessary for optimal neuroprotection in CNS injury, since it is their role to suppress autoimmune responses.

$T_{\text {reg }}$ in CNS injury: the simple

Early experiments, though lacking the sophisticated methods available today, hinted that the presence of $\mathrm{T}_{\text {reg }}$ cells limited the beneficial potential of autoimmune $\mathrm{T}_{\text {eff }}$ (Kipnis et al., 2002a). The techniques used in those studies did not rely on the targeting of specific molecules, given that they were performed at a time when the molecular characteristics of $\mathrm{T}_{\text {reg }}$ were just beginning to emerge. Although these techniques could not provide unequivocal answers, they were able to demonstrate a drop in $\mathrm{T}_{\text {reg }}$ numbers and an increase in the proportion of $\mathrm{T}_{\mathrm{eff}}$ to be associated with increased neuronal survival after CNS injury. As an example, a three-day postnatal thymectomy resulted in a decrease in $\mathrm{T}_{\text {reg }}$ cells relative to $\mathrm{T}_{\text {eff }}$ cells in adult mice, permitting increased post-injury neuroprotection compared to non-thymectomized controls (Kipnis et al., 2002a). Similarly, low-dose irradiation, which triggers apoptosis preferentially in $\mathrm{T}_{\text {reg }}$ cells and promotes proliferation of $\mathrm{T}_{\text {eff }}$ cells, increased neuronal survival after injury to either the rodent optic nerve or spinal cord and following glutamate-mediated toxicity of retinal ganglion cells (Kipnis et al., 2004b). Moreover, several compounds that decrease the number or activity of $\mathrm{T}_{\text {reg }}$ cells have also shown neuroprotective effects. Dopamine, which can decrease the suppressive ability of $\mathrm{T}_{\text {reg }}$ cells through ERK1/2 signaling, increases neuroprotection when administered systemically (Kipnis et al., 2004c). In a more immune-relevant paradigm, the synthetic, bacterial-DNA mimetic $\mathrm{CpG}$ modulated the regulatory capacity of $\mathrm{T}_{\text {reg }}$ cells, presumably by acting through Toll-like receptors, 
and increased neuronal survival by decreasing immune suppression (Johnson et al., 2007b). The results of these experiments collectively support the simple hypothesis that $\mathrm{T}_{\text {eff }}$ are required for neuroprotection after CNS injury and that this ongoing response is limited by $\mathrm{T}_{\mathrm{reg}}$. In line with this hypothesis, the limiting of the beneficial $\mathrm{T}_{\text {eff }}$ response by $\mathrm{T}_{\text {reg }}$ cells can be viewed as "the evolutionary compromise between a need and a risk" (Schwartz and Kipnis, 2002).

$T_{\text {reg }}$ cells in CNS injury: the complex

The real situation, however, is not always that simple and cannot always be

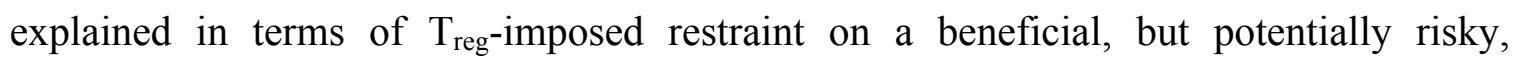
autoimmune response to injury. In Balb/c mice, transfer of splenocytes depleted of $\mathrm{CD} 25^{+}$cells into nude mice increased the beneficial effect on neuronal survival beyond the benefit that could be achieved by transferring whole splenocytes. In line with this, transfer of isolated $\mathrm{CD} 4{ }^{+} \mathrm{CD} 25^{+}$cells (i.e., $\mathrm{T}_{\text {reg }}$ cells) from naive mice into injured, immune-competent Balb/c mice partially abolished the beneficial neuroprotective effect (Kipnis et al., 2004a). However, transfer of the same $\mathrm{CD} 4{ }^{+} \mathrm{CD} 25^{+}$population resulted in the opposite phenotype when the recipient mice were of the C57/B6 background; that is, neuroprotection in the C57/B6 background was increased following injection of exogenous $\mathrm{T}_{\text {reg }}$ (Kipnis et al., 2004a). This finding was surprising because when the same two strains were subjected to immunization experiments with retina-specific antigens, neuronal survival in both strains was increased (Kipnis et al., 2004a). These results suggest that the nature of the endogenous response to injury in C57/B6 mice differs from that in $\mathrm{Balb} / \mathrm{c}$ mice and that $\mathrm{T}_{\text {reg }}$ manipulation might lead to neurodestruction or 
neuroprotection based on the genetic background of the recipient mouse. Interestingly, when $\mathrm{T}_{\text {reg }}$ cells were transferred into injured, immune-compromised mice, the treatment was beneficial in mice of both genetic backgrounds. Moreover, upon injection into an immune-deficient host these $T_{\text {reg }}$ cells not only lose their suppressive activity (Gavin et al., 2002) but also undergo proliferation and acquire effector function. It is therefore questionable whether these studies measure the effect of these injected $\mathrm{CD} 4{ }^{+} \mathrm{CD} 25^{+} \mathrm{T}_{\text {reg }}$ cells or the effect of the cells they differentiate into in immune-deficient hosts. Clearly, the nature of this " $\mathrm{T}_{\text {reg" }}$ " population requires further experimental clarification.

The findings described above raise concern with regard to the development of $T_{\text {reg- }}$ based therapies for clinical use. At this point it is obvious that we do not completely understand the complexity of $\mathrm{T}_{\text {eff }} / \mathrm{T}_{\text {reg }}$ interactions in situations involving CNS injury or neurodegenerative disorders. Therefore, instead of a rush to examine the protective activity of $T_{\text {reg }}$ cells under different neurodegenerative conditions, what is needed is a thorough study and systematic analysis of the interactions between the cellular participants to understand the complexity.

$T_{\text {reg }}$ cells in CNS injury: the confused I (strains and immunity)

As noted above, confusion still reigns with regard to $\mathrm{T}_{\text {reg }}$ function, and particularly to the protective properties of these cells under acute and chronic neurodegenerative conditions. Because most of the present knowledge in neuroimmunology comes from early studies on animal models of multiple sclerosis, both the commonly used cell lines and the acquired data are dominated by strains susceptible to autoimmune disease. Furthermore, because most existing transgenic and knockout mice are on a B6 
background, this is the most intensively studied strain in neuroimmunological investigations of CNS injury and chronic neurodegeneration. As described above, $\mathrm{T}_{\text {reg }}$ cells have opposite effects when transferred into Balb/c and C57Bl/6J mice, and their effects after injury in other strains are as yet unknown. This finding alone should raise questions among experimenters as to the most relevant animal model of CNS neurodegeneration for use in studies of the immune response. The use of several mouse strains in any study carried out to support the claims for a neuroprotective therapy or agent is probably necessary. The failure to transfer most of the proposed therapies from the laboratory to the clinic might be due in no small measure to a failure to follow this practice. Moreover, mice that are lymphopenic or otherwise immune-compromised might not be reliable models for addressing the role of $T_{\text {reg }}$ in neurodegeneration because $T_{\text {reg }}$ are changing their basic properties in immune-compromised hosts. The efforts at this stage should be aimed at understanding the immunobiology of $\mathrm{T}_{\text {reg }}$ cells under neurodegenerative conditions (both in immune-competent and immune-compromised hosts), before translation into human therapeutics is considered. At some point in the future we might then be justified in shifting the focus to clinical trials of $T_{\text {reg }}$-based neuroprotective therapies.

$T_{\text {reg }}$ cells in CNS injury: the confused II (anti-CD25 antibody)

The discovery that CD25 is highly expressed on $\mathrm{T}_{\text {reg }}$ cells led researchers to realize that the use of antibodies against this molecule would result in $T_{\text {reg }}$ cell clearance via the Fc $\gamma$ RIII-mediated mechanism (Setiady et al., 2010). Use of anti-CD25 antibody resulted in exacerbation of the infarct zone and functional deterioration in a stroke model 
of CNS injury (Liesz et al., 2009), leading to the conclusion that depletion of $\mathrm{T}_{\text {reg }}$ cells results in impaired recovery and that $T_{\text {reg }}$ are needed for neuroprotection after stroke. However, CNS injury results in presentation of self-antigens, leading to their activation and proliferation. Activated effector T cells express CD25, therefore, while CD25 serves as a marker for naturally occurring $\mathrm{T}_{\text {reg }}$ cells in naïve animals, it cannot distinguish between $\mathrm{T}_{\text {reg }}$ cells from activated $\mathrm{T}_{\text {eff }}$ cells in "challenged" mice, such as after CNS injury (because $T_{\text {eff }}$ cells that respond to injury will get activated and express CD25). Thus, mice that are treated with anti-CD25, which targets and depletes $\mathrm{T}_{\text {reg }}$ cells, will exhibit faster $T_{\text {eff }}$ response as well ( $T_{\text {eff }}$ cells get activated and proliferate more efficiently when $T_{\text {reg }}$ cells are removed). The anti-CD25 antibody, however, is cleared slowly and will target the emerging activated $\mathrm{T}_{\text {eff }}$ (expressing CD25) and result in their depletion as well. Therefore, exacerbation of CNS injury using anti-CD25 antibody does not necessarily imply that $\mathrm{T}_{\text {reg }}$ cells are needed for a better recovery; it might also suggest that activated $\mathrm{T}_{\text {eff }}$ cells, also eliminated by the antibody, are needed for neuroprotection. With the development of alternative tools to study the effect of $T_{\text {reg }}$ cell depletion, such as the expression of the diphtheria toxin receptor controlled by the Foxp3 promoter (Lahl et al., 2007), many of the experiments whose results have been equivocal because of this dual targeting of the anti-CD25 antibody (Liesz et al., 2009; Ren et al., 2010; Tenorio et al., 2011) need to be re-evaluated to determine the effect of each target individually. Furthermore, many of these studies have examined the immune response after complete depletion of regulatory $\mathrm{T}$ cells, creating a completely artificial system that can lead to autoimmune disorders (Wheeler et al., 2011). There is still little evidence on what the effects are of minor perturbations in the $\mathrm{T}_{\text {reg }}$ suppressive ability, rather than complete 
ablation of this important population. Additionally, while the downstream effectors are presumed to be effector $\mathrm{T}$ cells, this suppression $\mathrm{T}_{\text {eff }}$ activity after CNS injury, and where this suppression could be taking place, have still not been shown.

\section{Other immune cells}

While the cells that I have described above are seen in great numbers in the injured CNS and they have robust effects on the recovery from injury, there are several other immune subsets that exhibit a smaller presence in the injured tissue and have been less-well studied. Below is what we know about these other immune cells.

\section{Basophils/mast cells:}

Mast cells are found in the brain, especially in perivascular spaces and meningeal spaces. While little work has been done in traumatic CNS injury, mast cells do infiltrate into the injured CNS (Lozada et al., 2005), and one study showed that mast cell deficient mice exhibited greater proliferation of immune cells, an increase in astrogliosis, and increased neurodegeneration, suggesting a protective role for these cells post-injury (Hendrix et al., 2013).

\section{Eosinophils:}

Eosinophils are specialized cells that release cytotoxic granules to combat extracellular parasites. Despite their production of a molecule named eosinophil-derived neurotoxin 
(Durack et al., 1979), they have not been found in the injured CNS (Popovich et al., 1997a).

B cells

B cells are antibody-producing cells that have been implicated in autoimmune diseases such as systemic lupus erythematosis and neuromyelitis optica. In CNS tissue, they have been implicated in production of antibodies that help clear myelin. B cells accumulate in the injured tissue (Ankeny et al., 2006), and they seem to decrease the neuronal survival after injury (Schori et al., 2002; Schori et al., 2007), possibly due to their production of antibody (Ankeny et al., 2009).

\section{Immune signaling molecules}

The chief way that immune cells communicate with each other is through production of chemokines and cytokines, which are small proteins that are released and affect the function of the cells both in the injury milieu and systemically in the blood and lymphatic system. Here I will touch on some of the major cytokines in injury and how they affect the outcome.

\section{TNF}

TNF is one of the classical pro-inflammatory cytokines that is produced acutely after injury by microglia (Kita et al., 1997). TNF signals through two receptors: a ubiquitously expressed p55 receptor that promotes most of the cytokine production and apoptotic effects seen with TNF (Haviv and Stein, 1998) while an alternate p75 receptor 
is expressed on a smaller subset of cells and leads to protective effects (Kraft et al., 2009). Furthermore, TNF itself is found in two forms that can signal differently: membrane bound and soluble, which has been cleaved from the membrane and exists as a trimer. TNF can also induce death of cells, especially oligodendrocytes (Selmaj and Raine, 1988). It is also a major player in the recruitment of monocytes through the production of CCR2 (D'Mello et al., 2009). Finally, through TNF is usually considered a detrimental player in CNS injury due to its apoptotic effects, the final outcome may have a lot to do with when TNF is active. While TNF knockout mice actually display fewer deficits in the acute phases of injury, by 4 weeks they still display defects while control mice have completely recovered (Scherbel et al., 1999), possibly due to its role in axonal regrowth (Oshima et al., 2009). This suggests that while early TNF might in fact be detrimental, it is playing a different role in the remodeling process that occurs after injury.

\section{IL-1 $\beta$}

Very often, IL-1 $\beta$ is thought of in the same context of as TNF: a proinflammatory cytokine that mediates destructive effects. However, the story with IL-1 $\beta$ is not nearly as simple as with TNF. Indeed, as with the role of T cells in response to CNS injury, the role of IL-1 $\beta$ has not proved to be an easy question to answer, due to the fact that it does not act as a final effector of many of its functions, but rather acts as an initiating factor that causes further downstream changes that also mediate their own effects (Walsh et al., 2014). There are two signals that are needed to produce active IL$1 \beta$ : one to upregulate mRNA expression (usually ascribed to a TLR signal) and one to 
promote the processing of pro-IL-1 $\beta$ to an active form and promote its release (commonly through caspase activation by molecules such as ATP and uric acid, as well as a host of other factors reviewed in (Horvath et al., 2011)). This activation is done by the inflammasome, a recently discovered intracellular molecular complex that cleaves IL$1 \beta$ and several other IL-1 family members into an active form (Leavy, 2013). There are several sensor molecules that can initiate this caspase cleavage, which vary with respect to their tissue expression and ligand sensitivity. While astrocytes express a functional NLRP2 inflammasome (Minkiewicz et al., 2013) and neurons can process IL-1 $\beta$ through the NLRP1 inflammasome (de Rivero Vaccari et al., 2008), microglia are considered the primary producer of IL-1 $\beta$ in traumatic CNS injury (Herx et al., 2000). However, the role of IL-1 $\beta$ beyond this point is not entirely clear, and an abundance of both neuroprotective and neurodestructive functions have been ascribed to this important cytokine. Several studies have showed that IL-1 $\beta$ is a potent inducer of a reactive phenotype in astrocytes, promoting GFAP expression (Sticozzi et al., 2013), inducing proliferation (Pawliński et al., 2000), and, in line with the astrocytic role in re-establishing the BBB, promoting rapid sealing of the BBB (Herx and Yong, 2001; Scripter et al., 1997). (As a side note, IL-1 $\beta$ can paradoxically lead to a leak in the BBB by its action on microvascular endothelial cells in the absence of pathology (Rigor et al., 2012)). Further promoting neuroprotection, astrocytes treated with IL-1 $\beta$ are able to produce several neuroprotective neurotrophic factors, such as NGF (Spranger et al., 1990), CNTF (Albrecht et al., 2002; Herx et al., 2000), and FGF-2 (Albrecht et al., 2002). However, IL-1 $\beta$ can also promote neurodegeneration through its induction of secreted factors. Chief among these neurodestructive factors is TNF (Dunn et al., 2002) which, as discussed above, can 
directly lead to apoptosis of neurons through the p55 TNF receptor (Haviv and Stein, 1998). Additionally, astrocytes have been shown to mediate neuronal death after IL-1 $\beta$ treatment through expression of MMP9, which activates plasminogen through uPA (Thornton et al., 2008), a mechanism which has great relevance to stroke where additional exogenous tPA is commonly used as a treatment.

Furthermore, it has been shown that IL-1 $\beta$ has various effects directly on neurons, though most of these interactions have been only validated in vitro, and the relevance to the in vivo injury paradigm is still a matter of debate (Pinteaux et al., 2009). It has been shown by several groups that IL-1 $\beta$ does not cause any direct toxicity to neurons (Carlson et al., 1999; Viviani et al., 2003), but can modulate the effects of neurotoxic insults. Generally, low picomolar levels of IL-1 $\beta$ seem to exacerbate neuronal death, while higher nanomolar concentrations leads to protection (see references in (Pinteaux et al., 2009)), though there is a dependence on the type of insult used to induce death of neurons, as IL-1 $\beta$ can directly modulate the firing of neurons through GABA, NMDA, and AMPA receptors (Kawasaki et al., 2008).

Finally, although IL-1 $\beta$ has largely been considered to be a microglia-specific response, there is evidence that IL-1 $\beta$ production by cells other than the microglia could also play a role in injury. For instance, the NRLP1 inflammasome is activated in neurons after injury, and neutralization of this pathway with antibodies to ASC decreased inflammasome activation, leading to an improvement in outcome from injury (de Rivero Vaccari et al., 2008). This expression of IL-1 $\beta$ by non-immune CNS resident cells still needs further study to determine how it fits in with microglial IL-1 $\beta$ production in 
response to injury. However, there are some hints that IL-1 $\beta$ might not always be acting as a pro-inflammatory, neurodestructive cytokine as has been previously suggested. Recent work focusing on IL-4R $\alpha$ in aged mice show a decreased functional recovery from spinal cord injury; contrary to the belief that the loss of IL-4 would cause an inflammatory response, they surprisingly showed a decrease in IL-1 $\beta$ in both these models (Fenn et al., 2014). While this work did not focus on IL-1 $\beta$, it does beg the question of whether IL-1 $\beta$ is truly acting as a pro-inflammatory cytokine, or whether it simply behaves as an initiator of the immune response as we will discuss later.

\section{IL-10}

On the other side of the coin from TNF, IL-10 is a cytokine produced by many different immune cells acting on a variety of different cellular targets. IL-10 signaling, especially in the context of the immune response, is considered to be an antiinflammatory stimulus; IL-10 deficient mice develop spontaneous colitis characterized by an immune cell infiltration into the colon (but not the small intestine), weight loss, and systemic increases in pro-inflammatory cytokines (Kühn et al., 1993), and show an exacerbated phenotype in several other immune-driven pathologies (Iyer and Cheng, 2012). IL-10 promotes this anti-inflammatory signaling through the Jak1/STAT3 pathway, which prevents pro-inflammatory cytokine upregulation that would otherwise be robust, especially that of TNF, IL-1 $\beta$, and IL-6 (Strle et al., 2001). Because of the large variety of cells that produce and respond to IL-10, it is not surprising that there are several hypotheses about the action of IL-10 in CNS injury, which may not be mutually exclusive. Most of the studies have generally agreed that global treatment with IL-10 has 
proved to be a protective intervention in CNS injury (see references in (Thompson et al., 2013)). Moreover, while there are several cell types that are known to produce IL-10 after injury, the relative importance of each of these subsets in the protective response of IL-10 has not been established. Early work suggested that IL-10 production after injury occurred mainly in $\mathrm{CD}^{+} \mathrm{T}$ cells (of which $\mathrm{T}_{\text {reg }}$ cells are thought to be the main expressers (Frenkel et al., 2005)). However, further work showed that IL-10 is also expressed by monocytes that invade after injury, and is critical for the monocyte-mediated neuroprotective effect (Shechter et al., 2009). Finally, CNS-resident cells themselves also produce IL-10 that is important in neuroprotection after facial nerve axotomy (Xin et al., 2011). In fact, even adenoviral induction of IL-10 expression directly into neurons can lead to neuroprotection (Koeberle et al., 2004), suggesting that the cellular source of IL10 might not be important, given the redundancy of IL-10 expressing cells that are seen infiltrating the injury site after injury.

The cellular target of IL-10 has remained as enigmatic as its cellular source. Evidence from cultured cells suggests that IL-10 can signal directly to neurons to promote neuroprotection (Bachis et al., 2001) through production of anti-apoptotic factors and prevention of cytochrome c release (Zhou et al., 2009b). IL-10 has also been proposed to reduce detrimental cytokine expression in the injured spinal cord, in line with its well-established role as an anti-inflammatory cytokine (Bethea et al., 1999). However, questions still remain about the relevance of these findings in vivo, or even if IL-10 is also affecting the response to injury in other cell types such as astrocytes, OPC, oligodendrocytes, and other infiltrating immune cells after CNS injury. 


\section{TGF $\beta$}

As with IL-10, TGF $\beta$ is generally considered to be an anti-inflammatory cytokine, but demonstrates a much wider array of responding cell types and activities that it can elicit. Because of its ability to signal to many cell types, the precise role of TGF $\beta$ after injury is not well established. TGF $\beta$ plays several other roles in the healthy and injured CNS: it plays an important role as a signaling molecule in synaptic refinement (Bialas and Stevens, 2013), regulates microglial activity (Butovsky et al., 2013), and can regulate the adaptive immune response to CNS injury, inducing either regulatory T cells or Th17 cells (Bettelli et al., 2006b). One of the best-studied effects of TGF $\beta$ in CNS trauma is its role in induction of fibrosis (Logan et al., 1999), which has been of great interest because of the abovementioned axonal-inhibitory properties of ECM proteins produced after injury (Chen et al., 2000; Dickendesher et al., 2012) and the importance of re-establishing the $\mathrm{BBB}$ after trauma. Indeed, injured mice treated with exogenous TGF $\beta$ showed a decrease in iNOS, in line with its immunomodulatory phenotype, but an increase in scar formation at later time points (Hamada et al., 1996). In line with its barrier-forming function, neutralization of TGF $\beta 1$ caused cavitation in the injured spinal cord (King et al., 2004), but surprisingly the loss of TGF $\beta$ signaling in the injured CNS has not been shown to promote axonal outgrowth, as would be predicted in a model with decreased glial scar (Moon and Fawcett, 2001), suggesting that there are additional TGF $\beta$ independent factors that are produced by the glial scar that also inhibit axonal outgrowth. 


\section{IL-4}

IL-4 is a cytokine produced specifically by Th2 T cells, and signals through both the STAT6 pathway, through which it mediates its classical actions on the induction of Th2 T cells and B cells (Takeda et al., 1996a) and the IRS1/2 pathway, which plays an important role in cellular proliferation (Blaeser et al., 2003). Despite the fact that IL-4 has been known to promote allergic diseases and B cell proliferation for over 25 years (Finkelman et al., 1988), its role in neurodegeneration and neuroprotection is just now starting to emerge. The delay in study of IL-4 probably arises from early studies of cytokine expression in the spinal cord, which were unable to find increased expression of this potent cytokine (Uçeyler et al., 2008). While it was shown that IL-4 could modulate the function of microglia in vitro (Zhao et al., 2006), the first work to show that IL-4 was playing a role in CNS injury in vivo was published in 2010, when injection of anti-IL-4 antibodies lead to increased macrophage infiltration into the injury site through CCL2 expression and an increase in lesion size (Lee et al., 2010). Later work confirmed that IL4 is important for CCL2 expression and macrophage infiltration into the injury (Xiong et al., 2011), and further suggested that it promotes skewing towards an alternatively activated phenotype (Fenn et al., 2014; Zhao et al., 2012). With the potency with which IL-4 has been shown to act in vitro on the innate (Gordon and Martinez, 2010) and adaptive (Takeda et al., 1996a) immune systems and the drastic effects it can have in systems such as allergy and asthma (Paul and Zhu, 2010), it is surprising that there have been no studies that have examined this IL-4 mediated response in vivo. Among the many questions that have not yet been answered about this prototypical Th2 cytokine is 
whether there is upregulation of IL-4 at the site of the injury, the cells are producing and responding to it in vivo, and the functional outcomes of IL-4 signaling through either the STAT6 and IRS1/2 pathways.

\section{Alarmins in CNS injury}

With this complex immune responses occurring in response to CNS injury, perhaps it is a bit surprising that the initial signals that instruct the immune system to initiate its response in the first place have not yet been found. However, there is a class of signaling molecules that play this role in other injuries which could hold the key to this initiation of the immune response in the CNS: the alarmins.

Alarmins are molecules that are found pre-made or that are acutely upregulated in cases of tissue injury that initiate an immediate immune response to injury. They are the first molecules produced that initiate the immune response and play a vital role in instructing the downstream immune responses, both innate and immune. Coined by J. Oppenheim to originally describe anti-microbial peptides that activated immune cells cytokine production and chemotactic factors, the definition has now grown to include a wide variety of molecules mediators that are present hyper acutely after injury and initiate the immune response (Oppenheim et al., 2007). Here I will briefly describe some of the alarmins that have been described and their relevance to traumatic and inflammatory CNS injuries.

\section{The need for alarmins}

In light of the danger hypothesis, first suggested by Polly Matzinger in (Matzinger, 1994), the main purpose of the immune system is to be able to segregate 
salient from non-salient signals that it receives, rather than on self and non-self recognition. This hypothesis leaves room for autoimmune diseases (when immune recognition of self-antigens occurs in the presence of this danger signal), and gives a basis for how the immune system can respond to sterile injuries without infiltration of pathogens through epithelial barriers. While the danger signals that are released from pathogens that activate the immune system are numerous, and have been termed pathogen-associated molecular patterns (PAMPs), the analogous danger signals in response to the injured self are beginning to receive greater attention, and have been termed damage associated molecular patterns (DAMPs, another term for these alarmins). In the absence of an infection, the importance of distinguishing a salient stimuli is best exemplified by the difference in the response between programmed cell death by apoptosis and pathogenic cell death through trauma. Because programmed cell death is an important part of development and physiological functioning, the primary involvement of the immune system is clearing the debris so that an inflammatory secondary necrosis that could cause immune system-mediated bystander death is avoided. However, pathological cellular damage often requires much greater immune system involvement for an optimal outcome, and therefore would require the proper signals to initiate this immune cell activation.

This need to recognize which type of immune response is needed has been solved by the evolution of multiple redundant systems to sense molecules that are not released in apoptotic cell death, but signal that there has been a departure from normal physiological functioning. As it turns out, the body has adapted several redundant systems with which to segregate salient from non-salient stimuli, which I will discuss below. 


\section{Alarmins come in many shapes and sizes}

These alarmins encompass a class of molecules that are as diverse as their functions, but because they are filling the same signaling niche, they display several common properties: 1) They are not present extracellularly (or are present at low levels) in the absence of a stimulus that requires an immune system response, thus preventing a hypervigilance of the immune system that can lead to autoimmune disease. 2) They must be present in the tissue constitutively or be produced hyper acutely after a precipitating stimulus to ensure a rapid response to the stimulus. 3) Their receptors must be nonadaptive pattern recognition receptors to ensure that their important functions are conserved. 4) Finally, they must serve to activate the immune system through production of chemokines and cytokines in resident cells that will both serve to initiate the response in resident cells and will provide the cue for the circulating immune cells to infiltrate the tissue. As with other systems, evolution has not developed an entirely new set of signals to mediate this response, but rather has co-opted molecules that play an important role in the physiological functioning of the intact cell to provide for these functions in injury.

With this broad definition of an alarmin, and with the broad array of molecules that the immune system can recognize from pathogens, its not surprising that there are several large classes of molecules that can mediate these acute effects in sterile injuries. Probably the first, and best, characterized of these classes are intracellular proteins produced by the cell that are normally not seen in the extracellular space. These include IL-1 $\alpha$, IL-33, HMGB1, and the S100 class of proteins. Interestingly, most of these proteins exhibit either partial or complete nuclear localization in the living cell, ensuring 
that they will only be released into the extracellular space in high quantities upon breakdown of both the cytoplasmic and nuclear membrane.

The second class of alarmins is nucleic acids, and specifically mitochondrial DNA. While eukaryotic DNA has a characteristic methylation pattern that renders it nonimmunogenic, mitochondrial DNA has more similarities to prokaryotic DNA than of eukaryotic DNA (Zhang et al., 2010). Because of this, mitochondrial DNA is able to stimulate some of the same pathways that have been developed to sense pathogenic bacteria. Furthermore, this mtDNA can become even more immunogenic when it is oxidized, a condition that occurs under severe cell stress, endowing it with even greater specificity to damaged cells (Ding et al., 2013).

Finally there are small molecules that are released from the cell after death that promote immune system activation. While there are few molecules that have been described in this class of alarmin, they play a vital role in injury, especially in the CNS. The two molecules of this class that have been best studied are ATP and uric acid, both of which are purine metabolites. ATP is well known as the main energy source produced by oxidative phosphorylation and as a signaling molecule that can mediate diverse effects. Uric acid is a metabolite that is soluble intracellularly, but when exposed to the extracellular environment can precipitate and forms monosodium urate crystals. In excess, buildup of this metabolite is the causative agent of gout. Indeed, both of these molecules are unusual alarmins in many respects: Unlike the protein DAMPs, these small molecules tend to elicit weaker immune responses in the absence of other DAMPs, can be found outside the cell physiologically, and are well known to mediate their effects through mechanisms other than inflammatory cytokine and chemokine production. 


\section{Alarmin receptors}

As many of the same responses for the damage response are also required for the defense against pathogens, these systems have significant overlap between the receptors that they use for their signaling. Indeed, in the presence of a pathogen, this sensing of cellular damage will serve to further activate the immune response generated by PAMPs and amplify the protective response. However, in the absence of pathogens, such as in sterile injury, there is very often the need of an immune response to clear cellular debris, to provide support to the remodeling system, and to prophylactically ensure an immune cell presence, as there is often damage to the epithelial and mucosal membranes. The wound healing response has been particularly well-studied in this context of barrier tissues: the presence of the immune cell infiltration is necessary to undergo proper healing in these tissues (Leibovich and Ross, 1975), and blocking infiltration into the site of injury leads to delayed wound healing and larger injury areas (Suresh et al., 2012; Warren et al., 2004)

As compared to an adaptive immune response to self-peptides, the responses to these alarmins are evolutionarily conserved, suggesting an important role of these molecules. Chief amongst these receptors are the toll-like receptors (TLRs) and nod-like receptors (NLRs). TLRs are plasma membrane receptors that bind to ligands on the extracellular surface or in intracellular vesicles. This starts a signaling pathway through the adaptor molecules MyD88 or TRIF and ultimately leading to transcriptional upregulation of proinflammatory genes such as TNF and IL-6 through NFkb (for MyD88) and IFN-responsive genes such as CXCL10 through IRF3 (for TRIF). Because 
these receptors and their signaling have been reviewed extensively before (Kawai and Akira, 2005), we will not spend extensive time discussing them in this review. Nod-like receptors, on the other hand, are intracellular receptors that sense the presence of DAMPs in an as-of-yet unknown mechanism. This signal induces the consolidation of the inflammasome components, including caspase $1 / 11$, the adaptor molecule ASC, and the NLR protein itself. This receptor complex then signals through a proteolytic pathway to produce the active form of multiple mediators, the most well-known being IL-1 $\beta$ (Mariathasan et al., 2004).

While ATP is the best-studied NLR activator, many different substrates have been shown to activate these receptors, including uric acid, asbestos, silica, or even latex beads of defined sizes. Because of the wide variety of stimuli that is able to simulate these NLRs, it is thought that they are not directly binding to NLRs, but rather signal through an intermediate mechanism such as cell stress and altered intracellular ionic balance. For example, ATP signals through the P2Y class of G-protein coupled receptors and the P2X class of ionotropic receptors. While the signaling through the P2Y2 receptor is dispensable for inflammasome consolidation, the $\mathrm{P} 2 \mathrm{X} 7$ receptor, an ionotropic ATP receptor, is absolutely required. The dependence of ATP activation on this ion channel gives credence to the idea that NLRP3 activation goes through a metabolic/ionic stress pathway. The P2X7 receptor signaling has been shown to causes association of NLRP3 inflammasome components through an increase in sodium and calcium and a decrease in potassium, eventually leading to a disruption of mitochondria releasing oxidized DNA (Shimada et al., 2012). 
Interestingly, several alarmins that signal through NLRs and TLRs can also signal through alternate mechanisms that further enhances the immune response. ATP can cause alarmin-like responses independently of the $\mathrm{P} 2 \mathrm{X} 7$ receptor through the $\mathrm{P} 2 \mathrm{Y} 2$ metabotropic receptor. Knockout of this receptor prevents a migratory phenotype in microglia, which is important for their localization at the injury site (Davalos et al., 2005). This mechanism is shared between necrotic and apoptotic death and allows for a rapid induction of cell clearance (Elliott et al., 2009). HMGB1 is another alarmin that can signal through both a TLR and a non-TLR mediated mechanism. The receptor that mediates this non-TLR response to HMGB1 is the receptor for advanced glycation end products (RAGE), an immunoglobin-like receptor which signals through the Rho/Rac and MAPK pathways to promote migration, proliferation, and differentiation of cells, and, as with ATP, has been shown to play an important role in cellular migration (Degryse et al., 2001).

\section{Alarmin-induced chemokine and cytokine induction}

One of the characteristic features of alarmin signaling is the ability to upregulate chemokine production by resident cells to ensure a continued immune presence in the injury. In the CNS, most of the chemokine induction will occur in astrocytes and microglia, with neurons showing very little upregulation of NFKb, the primary driver of nuclear signaling for many DAMPs. While DAMPs increase chemokine expression, the pattern of chemokine expression varies between different alarmins. HMGB1 upregulated several chemokines in astrocytes, including the neutrophils chemoattractants CXCL1, CXCL2, and CCL3, as well as several chemokines that play a role in T cell trafficking, 
including CX3CL1, CCL2, CCL5, and CCL20 (Pedrazzi et al., 2007). Not surprisingly, then, HMGB1 has been shown to potentiate damage in several immune-mediated challenges through increased infiltration of neutrophils. In contrast, IL-33 upregulates CCL2, CCL3, CCL5, and CXCL10 production by microglia (Yasuoka et al., 2011), and the monocyte chemoattractant CCL2 in astrocytes (Kempuraj et al., 2013). With this chemokine repertoire that includes many $\mathrm{T}$ cell and monocyte chemoattractants, it is not surprising that IL-33 $3^{-/-}$mice show a deficit in monocyte infiltration into the injured spinal cord (Gadani, in preparation). ATP, while it can induce CCL3 production in microglia (Kataoka et al., 2009) and CCL2 in astrocytes (Panenka et al., 2001), seems to be playing a much larger role in injury by inducing cell migration through P2Y receptors, as previously described.

Not only do alarmins promote infiltration of the immune cells, but they also ensure that these cells become activated. The most common cytokines upregulated directly by alarmins are IL-6 and TNF, two cytokines directly regulated by alarmins that amplify the immune response and increase the infiltration and activation of peripheral immune cells. Both of these products are also upregulated in the CNS by ATP (Xia and Zhu, 2013), IL-33 (Hudson et al., 2008; Kempuraj et al., 2013), and HMGB1 (Laird et al., 2014; Okuma et al., 2014). Furthermore, many of these alarmins are able to increase activation of tissue resident macrophages, increasing their production of nitric oxide and their phagocytic ability (Yasuoka et al., 2011). Recent work has shown that excess mitochondrial DNA is able to promote innate immune responses and inflammatory migration and degranulation of neutrophils (Oka et al., 2012; Zhang et al., 2010). Uric acid acts on dendritic cells to increase their antigen presentation (Shi et al., 2003) and, as 
with many alarmins, can activate the inflammasome through NLRP3 to produce active IL-1 $\beta$ and IL-18 (Martinon et al., 2006).

Alarmins in CNS injury

$H M G B 1$

Despite the study that has gone into alarmins, most alarmins have not been wellstudied in the CNS, even in the context of traumatic injury where alarmins are traditionally thought to function. The notable exception that has received abundant attention in CNS development and injury is HMGB1. This molecule, which is found in the nucleus and acts as a non-DNA binding structural protein, is expressed during development (Hori et al., 1995), where it plays a role in cellular migration axonal outgrowth. While HMGB1 expression is largely downregulated in the adult brain, its expression reappears after spinal cord trauma (Chen et al., 2011). Similar to the improved functional recovery seen in both TLR4 deficient mice (Kigerl et al., 2007) and in RAGE knockout mice (Guo et al., 2014), inhibition of HMGB1 with antibody-mediated neutralization or with pharmacological blockers has been shown to lead to improvements in the recovery from both spinal cord injury (Zhai et al., 2012) and TBI models (Gu et al., 2014; Okuma et al., 2012). This HMGB1 signaling seems to act through both RAGE and TLR4 to promote this neurodestruction through separate mechanisms. Interaction with TLR4 on microglia leads to IL-6 expression, which subsequently signals to astrocytes to upregulate aquaporin-4, a water channel that mediates CNS edema after trauma to the CNS (Ribeiro et al., 2006). Depletion of RAGE in spinal cord injury, in contrast, seems 
to have a greater impact on the inflammatory response to injury, as seen by decreased infiltration of neutrophils and free radical production (Guo et al., 2014).

Purine metabolites: ATP and uric acid

Extracellular ATP is involved in a whole host of responses both physiologically and in pathology (reviewed in (Franke et al., 2012)). ATP plays an important role in the responses to CNS trauma that occur over the span of days, such as inducing astocytic gliosis. As seen with its role in inducing phagocyte migration towards apoptotic cells, ATP has been shown to be the most important molecule in microglia chemotaxis towards necrotic injury in vivo (Davalos et al., 2005). On the other end of the spectrum from ATP, which is involved in most every facet of the response to injury, is uric acid. Despite the fact that uric acid has been reported to promote antigen-specific responses through an interaction with DCs, it paradoxically decreased inflammation in EAE, an experimental model of multiple sclerosis, through an unrelated free radical scavenging phenotype. Whether endogenous uric acid is playing a role in promoting inflammatory diseases or the response to CNS injury has not yet been investigated.

$I L-33$

IL-33 has garnered much interest in the CNS. Despite the fact that IL-33 is able to upregulate the production of CCL2, TNF, and nitric oxide (Kempuraj et al., 2013), knockout of the receptor for IL-33 reduces the severity of EAE (Jiang et al., 2012). In line with this protective role for IL-33 in CNS injury, our lab recently showed that the alarmin IL-33 is essential for the protective monocytic infiltration into CNS injury, and 
its absence leads to both the decrease in infiltration of these cells and a deficit of the their tissue building anti-inflammatory skew. These deficits lead to a decreased functional recovery in necrotic insults in two CNS injury models, spinal cord injury and optic nerve crush injury, while having no effect in retinal glutamate toxicity (probably because IL-33 is not expressed in the retina; Gadani, et al., in preparation).

While these works have given an idea of whether alarmins are playing a beneficial or detrimental role after injury, none of them has been set up to answer the question of whether alarmins are indeed initiating the response to CNS injury initiating the immune response after injury. It could be that different alarmins control a the protective or destructive downstream pathways, and future work will determine whether promoting the protective and inhibiting the destructive pathways from the onset could set the immune system down the right path for the rest of the recovery process. 


\title{
Chapter III: Results
}

\section{AN ANTIGEN-INDEPENDENT IMMUNE RESPONSE TO CNS}

\section{INJURY}

\begin{abstract}
A body of experimental evidence suggests that $\mathrm{T}$ cells mediate neuroprotection following central nervous system (CNS) injury, although their antigen specificity and the precise mechanism underlying their beneficial effect are unknown. Here we provide compelling evidence that $\mathrm{T}$ cell-mediated neuroprotection after CNS injury can occur independently of major histocompatibility class II (MHCII) signaling to $\mathrm{T}$ cell receptors (TCRs). This antigen-independent response leads to MyD88-dependent Th2 induction by the damage associated molecular mediator IL-1 $\beta$, which is derived from microglia in the injured CNS tissue. T cell-derived IL-4 then directly protects and induces recovery of injured neurons via neuronal IL-4 receptors through potentiation of neurotrophin signaling. These findings shed a new light on the immune response to CNS injury and provide the first demonstration of a protective T-cell response induced by the molecular signature of the injured tissue independent of MHCII-TCR interactions. Our results point to IL-4 as a key immune molecule mediating neuroprotection and recovery of the injured CNS. These findings further illuminate the mechanisms for neuroprotection after CNS trauma, and have implications for the development of safe immune-based therapies for CNS injuries and neurodegenerative disorders.
\end{abstract}




\section{Results}

The accumulation of $\mathrm{T}$ cells in the injured CNS has been previously shown (Archambault et al., 2005), although what leads to T cell activation and the requirement for MHCII-TCR interaction for their neuroprotective phenotype are not well understood. Since autoimmune $\mathrm{T}$ cells can be destructive, such as in autoimmune diseases, we hypothesized that there may be an alternative protective signaling pathway in $\mathrm{CD} 4^{+} \mathrm{T}$ cells that would lead to a neuroprotective response to injury. To distinguish between antigen-specific and "alternative" activation of T cells after CNS injury, we first utilized major histocompatibility complex (MHC)-II knockout mice. Since MHCII is required for $\mathrm{CD}^{+} \mathrm{T}$ cell development, activation, and long-term survival, these mice do not contain conventional $\mathrm{CD}^{+} \mathrm{T}$ cells, but only a small population of $\mathrm{CD}^{+} \mathrm{T}$ cells with limited TCR diversity that recognize antigen in an antibody-like fashion (Tikhonova et al., 2012); in contrast, their $\mathrm{CD}^{+} \mathrm{T}$ cell and B cell repertoires are normal (Fig. S1). Prior to readministration of $\mathrm{T}$ cells into $\mathrm{MHCII}^{--}$mice, we examined their baseline spontaneous response to CNS injury. We used a well-established and highly reproducible mouse model of acute optic nerve crush injury to quantitatively determine the effects on survival of the parent cell bodies of optic nerve axons, i.e., the retinal ganglion cells (RGCs) at one week post-injury (Fig. 1a). As expected from previous studies with different $\mathrm{T}$ cell deficient animals (Kipnis et al., 2001), $\mathrm{MHCII}^{-1-}$ mice that are missing $\mathrm{CD}^{+} \mathrm{T}$ cells (Fig. 1b) exhibited reduced neuronal survival compared to background-matched wild type mice (Fig. 1c; contralateral retinas with uninjured optic nerves did not differ in RGC counts (Fig. 1d)). 
To determine if the lack of $\mathrm{CD} 4^{+} \mathrm{T}$ cells also affects functional recovery from CNS injury, we used a calibrated spinal cord contusion injury at the T9-T10 vertebra, and measured hind limb functional recovery with the Basso Mouse Scale (Basso et al., 2006). In line with the optic nerve injury experiments, $\mathrm{MHCII}^{--}$mice displayed worse functional recovery compared to their wild type counterparts (Fig. 1e). These results suggest that endogenous conventional $\mathrm{CD}^{+} \mathrm{T}$ cells are indeed vital for immunemediated neuroprotection and recovery after CNS injury.

To address our question whether antigen recognition by $\mathrm{T}$ cells is essential to their neuroprotective response, we transferred $\mathrm{CD}^{+} \mathrm{T}$ cells from naïve $\mathrm{C} 57 \mathrm{Bl} / 6$ mice into $\mathrm{MHCII}^{--}$mice. This transferred $\mathrm{CD}^{+}$population is unable to be maintained in the periphery due to the lack of MHCII in these mice; however, in line with previous reports (Takeda et al., 1996b), the cells survived for a week in the recipient mice (Fig. 1f, g). Surprisingly, mice that received $\mathrm{CD}^{+} \mathrm{T}$ cells displayed significantly more surviving neurons than vehicle treated $\mathrm{MHCII}^{-/}$mice lacking $\mathrm{CD}^{+} \mathrm{T}$ cells $(\mathbf{F i g} . \mathbf{1 h}$; RGC counts in contralateral retinas did not differ between the groups (Fig. S2a)). To determine if antigen specificity is also dispensable for $\mathrm{CD}^{+} \mathrm{T}$ cell-mediated functional recovery from spinal cord injury, we transferred $\mathrm{MHCII}^{-/-}$mice with $\mathrm{CD}^{+} \mathrm{T}$ cells from naïve $\mathrm{C} 57 \mathrm{Bl} / 6$ mice or vehicle at both one day after spinal cord contusive injury and 8 days after spinal cord injury. As with optic nerve injury, mice that received $\mathrm{CD} 4^{+} \mathrm{T}$ cells exhibited greater functional recovery from spinal cord injury than mice that were treated with saline (Fig. 1i) 
To confirm that the phenotype we observed was not due to activation of $\mathrm{CD} 4^{+} \mathrm{T}$ cells in their naïve host or due to adoptive transfer, we repeated the study using a model where $\mathrm{CD}^{+} \mathrm{T}$ cells were allowed to develop in vivo in $\mathrm{MHCII}^{-/-}$mice. To this end, we transplanted P2 thymi from wild type or $\mathrm{MHCII}^{-/}$donors under the kidney capsules of 3week-old $\mathrm{MHCII}^{-/}$recipients. These exogenous thymi were implanted 6 weeks before optic nerve injury to allow enough time for sufficient production of $\mathrm{CD}^{+} \mathrm{T}$ cells. In agreement with the previously published works (Nesić and Vukmanović, 1998), introduction of the wild type thymi allowed the $\mathrm{MHCII}^{--}$recipients to generate endogenous $\mathrm{CD}^{+} \mathrm{T}$ cells (Fig. 1j). Although wild type thymi-transplanted mice now had mature $\mathrm{T}$ cells, these cells were unable to interact with endogenous antigen presenting cells due to the MHCII deficiency in the host, and thus they were not able to respond to antigenic stimuli. $\mathrm{MHCII}^{-/-}$recipients of $\mathrm{MHCII}^{-/-}$thymi were unable to generate mature $\mathrm{T}$ cells and served as negative control. Once the mice had developed $\mathrm{CD} 4^{+} \mathrm{T}$ cells, they underwent optic nerve injury. Mice that received wild type thymi, and thus developed $\mathrm{CD}^{+} \mathrm{T}$ cells, displayed significantly more surviving neurons than the recipients of $\mathrm{MHCII}^{-/-}$thymi that did not possess $\mathrm{CD}^{+} \mathrm{T}$ cells (Fig. 1k; RGC counts in contralateral retinas did not differ between the groups (Fig. S2b)). These results further suggest that mature $\mathrm{CD} 4^{+} \mathrm{T}$ cells can exert a beneficial effect after sterile injury, even in the absence of recognition of their cognate antigen.

As an alternate method of studying the role of antigen-nonspecific T cells in CNS injury, we also examined neuronal survival in TCR-transgenic mice bearing only ovalbumin-specific $\mathrm{CD}^{+} \mathrm{T}$ cells on a $\mathrm{Rag} 1^{-/-}$background (OT-II/Rag $1^{-/-}$mice). These 
OT-II/Rag $1^{-/-}$mice, which lack the entire adaptive immune system except for a population of $\mathrm{CD}^{+} \mathrm{T}$ cells specific for ovalbumin (Fig. 11), exhibited an enhanced neuroprotective response after optic nerve crush compared to Rag $1^{-/-}$background controls (Fig. 1m; RGC counts in contralateral retinas did not differ between the groups (Fig. S2c)), further indicating that cognate antigen recognition is not prerequisite for $\mathrm{T}$ cells to acquire their neuroprotective properties after CNS injury.

In search for a better understanding of molecular mechanism underlying $\mathrm{T}$ cell mediated neuroprotection and recovery, we analyzed the cytokines that $\mathrm{T}$ cells from the injured spinal cord produced and found that one of the major cytokines produced by $\mathrm{T}$ cells at the site of injury was IL-4. Interestingly, we found that T cells in the site of injury produced more IL-4 than T cells from lymph nodes, and even more than $\mathrm{CD}^{+} \mathrm{T}$ cells isolated from the meninges (Fig. 2a, b), a compartment that is known for its IL-4 skewed environment (Baruch et al., 2013; Derecki et al., 2010). T cells from the injury site were the major source for IL-4 production as compared to any other cells in the injured CNS (Fig. 2c). To further confirm that IL-4 production in the spinal cord is due to $\mathrm{CD} 4^{+} \mathrm{T}$ cells, we examined cultured $\mathrm{CD}^{+} \mathrm{T}$ cells, microglia, astrocytes, and neurons for IL-4 expression. While Th0 cells in vitro expressed abundant Il4 mRNA, and this expression was further increased by culturing the cells in Th2 skewing conditions, there was a lack of Il4 expression by resident non-immune CNS cells, i.e. astrocytes, neurons, and oligodendrocytes (Fig. 2d).

Due to shared signaling pathways utilized by both IL-4 and growth factors such as neurotrophins and IGF1 (through IRS1/2 signaling (Blaeser et al., 2003; Reichardt, 
2006)) and since IL-4 has been previously implicated in the healthy (Derecki et al., 2010) and injured (Falcone and Bloom, 1997) CNS, it was reasonable to assume that it could be acting as a $\mathrm{T}$ cell derived neuroprotective molecule. To address the role of IL-4 on neuronal survival after CNS injury, we used again the optic nerve crush injury model to compare neuronal survival between IL- $4^{-/-}$mice and background matched wild type controls. IL-4 ${ }^{-/}$mice exhibited impaired neuronal survival as compared to their wild type counterparts (Fig. 2e; RGC counts in contralateral retinas did not differ between the groups (Fig. S2d)). Importantly, $\mathrm{CD}^{+} \mathrm{T}$ cell infiltration into the injured tissue was not affected in IL- $4^{-/-}$mice (Fig. 2f). To test whether the protective IL-4 is indeed produced by the immune cells, we transplanted IL- $4^{-/}$or wild type bone marrow into irradiationconditioned wild type hosts. After the bone marrow had fully engrafted (peripheral engraftment at $>90 \%$ ), mice underwent optic nerve injury. Recipients of $\mathrm{IL}_{-} 4^{-/-}$bone marrow demonstrated impaired neuronal survival as compared to control mice that received wild type bone marrow (Fig. 2g; RGC counts in contralateral retinas did not differ between the groups (Fig. S2e)). To further ensure that the neuroprotective IL-4 originated from $\mathrm{CD}^{+} \mathrm{T}$ cells, we injected acutely isolated IL-4-deficient $\mathrm{CD} 4^{+} \mathrm{T}$ cells (from IL-4 $4^{-/}$donors) or IL-4-sufficient $\mathrm{CD} 4^{+} \mathrm{T}$ cells (from wild type donors on the same genetic background) into Rag $1^{-/-}$(T cell deficient) mice and subjected these mice to optic nerve crush injury 3 weeks later. Wild type $\mathrm{CD}^{+} \mathrm{T}$ cells led to a higher neuronal survival in $\mathrm{Rag} 1^{-/-}$hosts than IL-4 ${ }^{-/-} \mathrm{T}$ cells $($ Fig. $2 \mathrm{~h}$; RGC counts in contralateral retinas did not differ between the groups (Fig. S2f)), suggesting that the primary source of neuroprotective IL-4 after injury is indeed the $\mathrm{CD} 4^{+} \mathrm{T}$ cells. 
To determine if the neuroprotective effect of IL-4 would also impact functional recovery, we performed spinal cord injury on IL $-4^{-/-}$mice and wild type controls. As expected from the optic nerve injury model, $\mathrm{IL}-4^{-/-}$mice displayed a deficit in functional recovery from spinal cord injury (Fig. 2i). Additionally, we transferred wild type or IL-4 ${ }^{-}$ ${ }^{1-} \mathrm{CD}^{+} \mathrm{T}$ cells in to $\mathrm{Rag} 1^{-/-}$mice. The $\mathrm{T}$ cells were allowed to engraft in the recipients for 3 weeks before performing spinal cord injury. $\operatorname{Rag} 1^{-/-}$mice that received wild type $\mathrm{T}$ cells exhibited enhanced functional recovery from spinal cord injury than those that received IL- $4^{-/} \mathrm{CD} 4^{+} \mathrm{T}$ cells, confirming that a neuroprotective $\mathrm{CD} 4^{+} \mathrm{T}$ cell response is IL-4 mediated (Fig. 2j).

Two mechanistic questions remained unanswered: what induces $\mathrm{T}$ cells at the site of injury to express IL-4 and how does IL-4 mediate neuroprotection and recovery?

To address the mechanism underlying the Th2 skew at the site of injury, we established an in vitro system where $\mathrm{CD}^{+}$lymphocytes from cervical lymph nodes were incubated with isolated optic nerves (in the presence of the $\mathrm{CD}^{-}$fraction from the lymph node serving as antigen presenting cells). Because our findings indicated that the induction of a neuroprotective T cell response does not require MHCII-TCR interactions (Figure 1), we postulated that soluble factors from the injured CNS induce a Th2 phenotype directly on $\mathrm{T}$ cells. To assess $\mathrm{Th} 2$ skewing in wild type $\mathrm{T}$ cells, we measured a master transcriptional regulator of Th2 cells, Gata3 (Zheng and Flavell, 1997) by realtime quantitative PCR. Isolated $\mathrm{CD}^{+}$lymphocytes were incubated with $\mathrm{CD}^{-}$APCs and injured optic nerves for 3 days, after which an increase in Gata3 in $\mathrm{CD}^{+} \mathrm{T}$ cells was evident (Fig. 3a), suggesting a Th2 skew by factors within the CNS tissue. Although 
Gata3 is a master regulator of Th2 skew, we also assessed more direct measurements of IL-4 in these cells. To this end, we first used T cells from reporter KN2 mice, which express human CD2 indicating IL-4 protein translation (Mohrs et al., 2005). Incubation of KN2-derived T cells with injured CNS tissue indeed showed an increase in T cellderived IL-4 production (Fig. 3b, c). Additionally, intracellular staining for IL-4 in cultured $\mathrm{T}$ cells, which directly assesses IL-4 production but requires the direct analysis of IL-4 protein, which is produced at low levels (hence the reporter mice were established), also demonstrated an increase in IL-4 production upon incubation with optic nerves (Fig. 3d, e) and a concurrent decrease in IFN- $\gamma$ production (Fig. 3f), suggesting that the optic nerve tissue is sufficient to drive a Th2 response in $\mathrm{CD} 4^{+} \mathrm{T}$ cells.

Since we detected a correlation between IL-4 expression by $\mathrm{T}$ cells and their Gata3 expression, and due to a robustness of Gata3 assessment by qPCR, we proceeded with our studies to identify the "factor" that leads to IL-4 production using Gata3 as an expression readout. Of note, optic nerves isolated from 3-day optic nerve-injured or naïve mice resulted in similar degrees of Gata3 induction in T cells (Fig. 3g), suggesting that the factor/s is/are produced acutely after injury caused by excision of the optic nerves. To determine if additional "damage" to the nerve is necessary to promote this phenotype or if removal from the mouse causes sufficient damage to drive the Th2 response, we cultured nerves that had been minced or left intact, and found that the damage to the axons from removal of the nerves from their hosts was sufficient to drive the Gata3 induction in $\mathrm{CD}^{+} \mathrm{T}$ cells $(\mathbf{F i g}$. $\mathbf{3 h})$. 
As we did not see a requirement for antigen specificity of $\mathrm{CD}^{+} \mathrm{T}$ cells in order for them to become protective in vivo, we asked if antigen specificity played a role in this Th2 skew in vitro. Therefore, we first cultured $\mathrm{T}$ cells with myelin, which contains many of the antigens that have been implicated in T cell autoimmunity to the CNS. However, purified myelin did not have any effect on Gata3 expression in $\mathrm{CD}^{+} \mathrm{T}$ cells (Fig. 3i). Since in vivo MHCII recognition was not needed for the $\mathrm{T}$ cell-mediated beneficial response, we replaced the wild type $\mathrm{CD}^{-}$antigen presenting cells with $\mathrm{MHCII}^{-/-}$antigen presenting cells in our cultures of wild type T cells to determine if MHCII signaling on antigen presenting cells was necessary for the induction of Th2 skewing by CNS tissue. Indeed, MHCII deficiency on antigen presenting cells did not affect Gata3 induction in $\mathrm{CD}^{+} \mathrm{T}$ cells in response to incubation with the injured optic nerve (Fig. $\left.3 \mathbf{j}\right)$. Similarly, T cells derived from OTII $/ \operatorname{Rag} 1^{-/-}$mice and, therefore, not responding to any of the antigens present in the injured tissue, also demonstrated upregulation of Gata3 after incubation with the injured optic nerves (Fig. 3k). Moreover, removal of antigen presenting cells from the cultures of $\mathrm{T}$ cells with the optic nerves did not affect the induction of Gata3 in T cells (Fig. 31), suggesting that the factor originating from the injured CNS interacts directly with $\mathrm{T}$ cells and skews them to the Th2 lineage. To determine if there was a cellular interaction that was causing this upregulation of Gata3 or if there was a soluble factor that was mediating the effects of optic nerves on $\mathrm{T}$ cells, we cultured $\mathrm{CD}^{+} \mathrm{T}$ cells with optic nerves that were separated from the $\mathrm{T}$ cells by 0.4 $\mu \mathrm{m}$ transwell. Optic nerves were able to upregulate Gata3 mRNA in $\mathrm{CD}^{+} \mathrm{T}$ cells, even through transwell separation (Fig. 3m), suggesting that soluble factors originating in the injured CNS were responsible for this effect. To confirm this phenotype, we conditioned 
media with optic nerve explants for 3 days. The media was then added to $\mathrm{T}$ cell cultures after being filtered through a $0.22 \mu \mathrm{m}$ filter. As expected, conditioned media was also able to induce the upregulation of Gata3 in T cells (Fig. 3n). To rule out that possibility that the optic nerves could be producing IL-4 themselves to mediate this effect, we used optic nerves from IL- $4^{-/-}$mice, and a similar degree of Gata 3 induction was achieved (Fig. 3o).

We hypothesized that the Th2 skewing is induced via molecular patterns/mediators or 'alarmins' (Oppenheim et al., 2007) originating from the damaged tissue. Pattern recognition is mediated by unique receptors, such as Toll-like receptors (TLR), among other pattern recognition receptors, which are highly expressed on immune cells (Pasare and Medzhitov, 2004). Many TLRs signal through a common signaling molecule, MyD88, which mediates downstream transcriptional activation by several mechanisms (Kawai et al., 1999). To address a possible role of MyD88 signaling in CNS-induced Th2 skewing, we used $\mathrm{T}$ cells from wild type or MyD88 ${ }^{-/}$mice incubated with the optic nerves. Whereas T cells from wild type mice demonstrated, as expected, upregulation of Gata3, no such upregulation was detected in the cultures with MyD88 $^{-/-} \mathrm{T}$ cells (Fig. 4a), suggesting that the signaling pathway that results in Th2 skewing is mediated, at least in part, via MyD88 intrinsic to T cells.

To determine if the myriad of molecular 'alarmins' that are released and signal from the injured CNS induce a desired neuroprotective $\mathrm{T}$ cell response in vivo, we isolated $\mathrm{T}$ cells from the injured spinal cord of wild type and $\mathrm{MyD}^{-{ }^{-/}}{ }^{-}$mice and looked at intracellular cytokine expression. While there was high expression of IL-4 in wild type 
$\mathrm{T}$ cells, MyD88 ${ }^{-/-}$mice exhibited lower amounts of IL-4. Of note, the IL-4 expression was not completely eliminated in MyD88 $8^{-/-}$mice, suggesting that there are other MyD88independent pathways that can induce IL-4 expression after spinal cord injury. To examine whether MyD88 signaling is physiologically relevant to a neuroprotective immune response after injury, we examined optic nerve injury in $\mathrm{MyD} 88^{-/-}$mice and found that their neuronal survival was indeed significantly impaired compared to control mice (on identical genetic background with sufficient MyD88 expression) (Fig. 4c; RGC counts in contralateral retinas did not differ between the groups (Fig. S2g)). Bonemarrow transplantation from MyD88 $8^{-/}$or wild type donors into irradiation-conditioned wild type recipients recapitulated the results obtained with germline knockout mice (Fig. 4d; RGC counts in contralateral retinas did not differ between the groups (Fig. S2h)), further pointing the importance on the immune cells as responders to MyD88-dependent signals.

To determine what factor(s) might be playing a role in inducing Th2 skewing after CNS injury, we tested a panel of MyD88-dependant TLR ligands. Much to our surprise, none of the tested TLR ligands increased Gata 3 in T cells, and several decreased its expression instead (Fig. S3). Therefore, we tested an important family of cytokines (IL-1 family), which signals in MyD88-depenent manner through IL-1R (Burns et al., 1998), focusing on whether IL-1 $\beta$ leads to IL-4 induction in T cells after injury. Although counterintuitive, this "pro-inflammatory" cytokine has been previously linked to induction of anti-inflammatory response (Humphreys and Grencis, 2009; Schmitz et al., 2003) and has recently been linked to induction of $\mathrm{T}_{\text {reg }}$ in the gut (Mortha et al., 2014). 
To test the role of the IL-1 family of cytokines we again used our culture model. Addition of IL-1 $\beta$ to T cell cultures resulted in an upregulation of Gata3 in wild type T cells (Fig. 4e) but not in MyD88 $8^{-/-} \mathrm{T}$ cells (Fig. 4f). In contrast, the closely related family member IL-1 $\alpha$ produced a small but non-significant increase in Gata3 when cultured with $\mathrm{T}$ cells at the same concentrations as IL-1 $\beta$ (Fig. $\mathbf{4 g}$ ). Similar results were obtained with recombinant IL-33, another member of the IL-1 family of cytokines (data not shown). Furthermore, when T cell cultures were treated with IL-1 $\beta$, an increase in IL-4 protein produced by $\mathrm{T}$ cells was evident by intracellular labeling (Fig. $4 \mathbf{h}, \mathbf{i})$, along with a decrease in IFN- $\gamma($ Fig. 4j), similar to what was achieved upon incubation with the optic nerve tissue (Fig. 3d-f).

We next confirmed that IL-1 $\beta$ was present in the injured CNS, as has been previously published (Allan et al., 2005; de Rivero Vaccari et al., 2008). Indeed, $I l 1 b$ mRNA was dramatically increased in optic nerve tissue after injury (Fig 4k). To determine if IL-1 $\beta$ was playing a role in CNS injury, we again established bone marrow chimeras with $\mathrm{C} 57 \mathrm{~B} 1 / 6$ or $\mathrm{IL}-1 \beta^{-/}$bone marrow donors transplanted into $\mathrm{C} 57 \mathrm{Bl} / 6$ recipients that had been lethally irradiated with their heads uncovered, allowing engraftment of monocytes into the brain (to yield microglia-like cells (Derecki et al., 2012); Fig. 4I). Mice that received IL-1 $\beta^{-/}$bone marrow displayed a decrease in neuronal survival as compared to wild type mice (Fig. $\mathbf{4 m}$ ), suggesting that IL-1 $\beta$ produced by the transplanted cells were playing a beneficial role in CNS injury.

To examine which cells might be producing IL-1 $\beta$, we examined microglia, which are partially replaced in the optic nerve upon irradiation with the head exposed 
(Fig. 4I). To determine if these microglia could be the source of IL-1 $\beta$ in injury, we isolated myeloid cells from the injured or uninjured CNS, and examined their production of IL-1 $\beta$. The $\mathrm{CD} 5^{+} \mathrm{CD} 11 \mathrm{~b}^{+}$myeloid cells from injured mice produced IL-1 $\beta$ (Fig. $4 \mathbf{n}$, o), while $\mathrm{CD} 45^{+} \mathrm{CD} 11 \mathrm{~b}^{+}$cells from uninjured mice and $\mathrm{CD} 45^{-}$cells from both injured and uninjured mice did not produce IL-1 $($ Fig. 4o).

To further implicate IL-1 $\beta$ production as an important player in optimal recovery from CNS injury, we tested the role of the NLRP3 inflammasome, which is important in processing pro-IL-1 $\beta$ to its mature form in myeloid cells (Kanneganti et al., 2006). In the CNS, the NLRP3 inflammasome has been shown to mediate IL-1 $\beta$ production in microglia, while the NLRP1 and NLRP2 inflammasomes are important in IL-1 $\beta$ production by astrocytes and neurons (de Rivero Vaccari et al., 2008; Hafner-Bratkovič et al., 2012; Minkiewicz et al., 2013; Pan et al., 2014; Tomura et al., 2012). To this end, we performed optic nerve injury on C57B1/6 mice that had been transplanted with either $\mathrm{NLRP}^{-/-}$or $\mathrm{C} 57 \mathrm{Bl} / 6$ bone marrow. As with mice that received IL- $1 \beta^{-/-}$bone marrow, transfer of NLRP3 ${ }^{-/-}$bone marrow lead to a decrease in neuronal survival (Fig. 4p), further implicating the importance of IL-1 $\beta$ production by microglia in recovery from CNS injury.

Finally, to directly demonstrate that microglia are, indeed, the cells that produce IL-1 $\beta$ after injury, we repeated the bone marrow chimeras, but this time shielded the heads during irradiation to prevent bone-marrow derived monocyte engraftment in the CNS (Mildner et al., 2007; Shechter et al., 2009). In contrast to mice that received IL-1 $\beta^{-}$ ${ }^{\prime-}$ bone marrow after head-exposed irradiation (Fig. 4m), those that received IL-1 $\beta$ bone 
marrow after head-shielded irradiation did not display any difference in neuronal survival (Fig. 4q), suggesting that production of IL-1 $\beta$ by microglia at the site of injury is mediating its neuroprotective effects.

Lastly, to address the molecular mechanism of IL-4 mediated beneficial impact on injured neurons, we utilized a well-established and robust ex vivo axonal outgrowth model using cortical explant (EC) cultures, allowing for the analysis of soluble factors on outgrowth in an organotypic setting (Woodhams and Atkinson, 1996) (Fig. 5a). This model, while not an in vivo system, better mimics the in vivo condition than isolated neuronal cultures lacking the glial compartment, which is of importance for proper axonal outgrowth. Furthermore, this system mimics injury, as the physiological connections of the entorhinal cortex need to be severed in order to produce the explant cultures, and thus the outgrowth is an effort of the damaged neurons to reconnect to their targets. IL-4-producing Th2 cells obtained by an antigen-independent stimulation protocol (Con-A activation) and kept in a collagen matrix next to the slice increased axonal outgrowth in this ex vivo model, whereas Th1 cells failed to do so (Fig. 5b). In order to define the impact of IL-4 on axons, induction of outgrowth by Th2 cells served as a readout assay for our further analyses. In fact, axonal outgrowth achieved by Th2 cells was inhibited by the addition of an IL-4 neutralizing antibody (Fig. 5c), but remained unaffected by neutralization of either IL-10 or IL-13 (Fig. 5d), strongly suggesting for IL-4 as a key Th2-derived molecule that mediates the effect. To ensure that this response was specific to IL-4 signaling on the EC slices, we examined Th2 cells co-cultured with EC slices from IL-4R ${ }^{-/}$mice. In contrast to control EC slices, Th2- 
skewed cells were not able to induce axonal outgrowth in slices from IL-4R ${ }^{-/-}$mice (Fig. 5e).

To further demonstrate that IL-4-induced outgrowth after lesion in the ex vivo model results in sprouting into the proper target tissue, we cultured EC slices from actin $^{\mathrm{GFP}}$ mice with wild type hippocampal slices (Hechler et al., 2010) to determine if application of recombinant IL-4 would increase the regrowth of axons from the EC through their physiological route, the perforant path, into the hippocampus (Fig. 5f). Indeed, exogenous IL-4 was able to potentiate axonal growth ( $\mathrm{GFP}^{+}$axons) into the hippocampal slices (Fig. 5g)

To determine if IL-4 could be signaling directly to neurons, we first examined whether neurons express the correct receptor for binding IL-4. In cultured neurons, IL$4 \mathrm{R} \alpha$ can be found as mRNA and protein (Fig. 5h, i). We next examined whether IL-4 could directly signal to neurons to promote axon elongation using cultures of cortical neurons which were treated with IL-4. Indeed, treatment of neuronal cultures with IL-4 promoted enhanced elongation of axons of compared to cultures treated with vehicle (Fig. S4), suggesting that IL-4 signaling directly to neurons could be promoting this Th2driven axonal outgrowth seen in our slice cultures. To test this hypothesis, we cultured Th2 cells with wild type or IL- $4 \mathrm{R}^{f l f f}$ :CamKII ${ }^{\text {cre }}$ slices (in which only neurons are deficient in IL-4R, while other neural cells in the slices are expressing their regular levels of IL4R). Whereas slices from the wild type mice showed, as expected, axonal outgrowth in response to Th2 (but not Th1) cells, slices from IL-4R ${ }^{\text {fllft }}$ :CamKII ${ }^{\text {cre }}$ showed no such 
response to Th2 cells (Fig. 5j), indicating that IL-4 signals directly through neuronal IL4R.

As mentioned above, IL-4 can act via a signaling pathway shared with growth factors, including neurotrophins, through IRS family of adaptor proteins (Blaeser et al., 2003; Reichardt, 2006) influencing signal transduction through AKT and MAPK signaling. Interestingly, a previous study showed no indications for a relevant role of endogenous neurotrophins in the initiation of axon outgrowth from cortical explants. However, application of recombinant NT-3 potentiated this spontaneous outgrowth (Hechler et al., 2010), pointing to a role of neurotrophins in strengthening the signaling in the pre-activated axonal growth pathway. To determine if this potentiation of neurotrophin signaling is involved in IL-4 mediated axonal outgrowth, we tested axonal outgrowth induced by Th2-cells after blocking the neurotrophin signaling. Indeed, we found that inhibitory antibodies to neurotrophins involved in outgrowth from EC-cultures (Prang et al., 2001) were effective in abolishing Th2-mediated axonal outgrowth (Fig. $5 \mathbf{k})$.

To determine if there is crosstalk between IL-4 and neurotrophin signaling at the level of the AKT and MAPK pathways in neurons, we tested whether IL-4 pretreatment would potentiate this pathway in neutrotrophin-treated neurons. Indeed, pre-treatment with IL-4, elicited an increase in MAPK signaling in neurotrophin-treated cultures, as evidenced by an increase in pMAPK44 to total MAPK44 (Fig. 5l, m). This effect was not present when IL-4R $\mathrm{R}^{-/-}$neurons were treated with IL-4 before neurotrophins were applied (Fig. 5l, n) Furthermore, blocking the AKT and MAPK signaling pathways downstream of IL-4R 
(Sun et al., 1995) and neurotrophin signaling (Zweifel et al., 2005), also abolishes the Th2 induced outgrowth of axons in EC slice cultures (Fig. 5o).

To determine if IL-4 could be a relevant treatment for CNS injury, we examined whether the IL-4R is expressed on the relevant CNS neurons in vivo. As demonstrated in cultured neurons, expression of the IL-4Ra is detectable on spinal axons, including those of the corticospinal tract (CST, Fig. 5p).

To determine if the IL-4 induced axonal outgrowth and IL-4R expression in the CST could be harnessed therapeutically, we injected Th2 cells directly into the site of injury, with PBS and Th1-injected mice serving as controls, and assayed the mice for axonal outgrowth and functional recovery. The locally injected $\mathrm{T}$ cells survived in the spinal cord, and could be seen even $5 \mathrm{~mm}$ beyond the injection site (Fig. S5). Despite the fact that wild type mice already display a highly-Th2 skewed environment in the injured spinal cord (Fig. 2a-c), addition of exogenous Th2-skewed cells, but not Th1 skewed cells, was able to further potentiate axonal outgrowth of CST axons $5 \mathrm{~mm}$ distal to the injury site (Fig. 5q, r) and to promote a significant increase in functional recovery from spinal cord injury (Fig. 5s) . 


\title{
THE ROLE OF REGULATORY T CELLS IN CNS INJURY
}

\begin{abstract}
Previous research investigating the roles of effector $\left(T_{\text {eff }}\right)$ and regulatory $\left(T_{\text {reg }}\right) T$ cells after acute injury to the central nervous system (CNS) has yielded contradictory conclusions, with both protective and destructive functions being ascribed to each of these T-cell subpopulations. Here we study this dichotomy by examining how regulation of the immune system affects the response to CNS trauma. We show that in response to acute CNS injury, both $\mathrm{T}_{\text {eff }}$ and $\mathrm{T}_{\text {reg }}$ subsets in the CNS-draining deep cervical lymph nodes are activated, and that surgical resection of such lymph nodes results in impaired neuronal survival. Depletion of $\mathrm{T}_{\text {reg }}$ cells, not surprisingly, induces a robust $\mathrm{T}_{\text {eff }}$ cell response in the draining lymph nodes and is associated with impaired neuronal survival. Interestingly, however, injection of exogenous $\mathrm{T}_{\text {reg }}$ cells, which limits the spontaneous beneficial immune response after CNS injury, also impairs neuronal survival. We found that no $\mathrm{T}_{\text {reg }}$ cells accumulate at the site of CNS injury, and that changes in $\mathrm{T}_{\text {reg }}$ cell numbers do not alter the amount of infiltration by other immune cells into the site of injury. The phenotype of macrophages at the site, however, is affected: both addition and removal of $\mathrm{T}_{\text {reg }}$ cells negatively impact the numbers of macrophages with alternatively activated (tissue-building) phenotype. Our data demonstrate that neuronal survival after CNS injury is impaired when $\mathrm{T}_{\text {reg }}$ cells are either removed or added. With this exacerbation of neurodegeneration seen with both addition or depletion of $\mathrm{T}_{\text {reg }}$ cells, we recommend exercising extreme caution when considering the therapeutic targeting $\mathrm{T}_{\text {reg }}$ cells after CNS injury, and possibly in chronic neurodegenerative conditions.
\end{abstract}




\section{Results}

\section{A CD4 ${ }^{+}$T cell response in the CNS-draining deep cervical lymph nodes after CNS injury}

To determine where the immune response to CNS injury was occurring, we first examined CNS draining deep cervical lymph nodes (dCLN) as compared to skin draining lymph nodes (SDLN; axillary and inguinal) for T cell activation and proliferation upon CNS injury. We found an increase in the number and percentage of CD4 ${ }^{+} \mathrm{T}$ cells and a concurrent reduction in the percentage of $\mathrm{CD}^{+} \mathrm{T}$ cells in CNS-draining deep cervical lymph nodes (Fig. 6a-c). No change in the number or percentage of $\mathrm{CD}^{+} \mathrm{T}$ cells was observed in the skin-draining lymph nodes (Fig. 6d-f). When the induced $\mathrm{CD}^{+} \mathrm{T}$ cells were examined for sub-population $\left(\mathrm{T}_{\text {reg }} v s . \mathrm{T}_{\text {eff }}\right)$, both activated $\mathrm{T}_{\text {eff }}\left(\mathrm{CD} 4^{+} \mathrm{CD} 25^{+}\right.$Foxp $\left.^{-}\right)$ and $\mathrm{T}_{\text {reg }}\left(\mathrm{CD}^{+}{ }^{+}\right.$Foxp3 $\left.^{+}\right)$cells were increased in the dCLNs after the injury (Fig. 6g-i), but not in the skin-draining lymph nodes (Fig. 6j-1). To determine if the immune response in the deep cervical lymph node was playing an important role in the response to CNS injury, we used an optic nerve injury model, where retinal ganglion cells are pre-labeled with the neuronal tracer Fluoro-gold then the optic nerve is injured and the number of surviving retinal ganglion cells (RGCs) in the retina are quantified (Fig. 6m). This injury leads to a decrease in the number of retinal ganglion cells in mice that underwent the deep cervical lymph node removal than those that received a sham surgery (Fig. 6n), while their contralateral uninjured retinas did not display a loss of retinal ganglion cells (Fig. 6o).

To determine if $\mathrm{T}$ cells from the injured dCLN displayed a different phenotype after CNS injury than the SDLN, we used flow cytometry to analyze the intracellular 
cytokines produced in the lymph nodes after injury. T cells from the dCLN displayed higher levels of IL-4 after optic nerve injury than those from the SDLN (Fig. 7a, b). To determine if $\mathrm{T}$ cells induced after optic nerve injury in the draining lymph nodes are capable of supporting alternative activation of macrophages, we isolated $\mathrm{T}$ cells from injured and uninjured dCLNs and SDLNs and co-cultured them with a pure population of bone-marrow derived macrophages. $\mathrm{T}$ cells from the deep cervical lymph nodes of optic nerve-injured mice were able to support an alternative activation phenotype of bone marrow macrophages in vitro, while $\mathrm{T}$ cells obtained from SDLNs of injured mice or from dCLNs of uninjured mice were unable to promote this alternatively activated (tissue-building) phenotype of macrophages (Fig. 7c). This suggests that the injury indeed induces $\mathrm{T}_{\text {eff }}$ cells in the draining $\mathrm{dCLN}$ that are capable of promoting a neuroprotective macrophage phenotype.

Because we observed that $\mathrm{T}$ cells in the draining lymph node were able to promote an alternative activation of macrophages, we addressed a possibility that $\mathrm{T}$ cells are controlling the phenotype of the infiltrating monocytes/macrophages. Arginase-1 expressing macrophages (M2-type) have been previously described to support neuronal survival after CNS injury (David and Kroner, 2011; Fenn et al., 2014; Kigerl et al., 2009; Shechter et al., 2013). Indeed, using immunohistochemistry of injured optic nerves we demonstrate that arginase-1 expression is induced in the injury site (Fig. 7d), whereas there is no detectable arginase-1 staining in the uninjured optic nerves (Fig. S6a). We established GFP $\Rightarrow \mathrm{C} 57 \mathrm{Bl} / 6$ bone marrow chimeric mice (Shechter et al., 2009), whose peripheral immune system is replaced by the $\mathrm{GFP}^{+}$bone marrow, but which have a 
significant number of $\mathrm{GFP}^{-}$microglia in the optic nerve (Fig. S6b) to distinguish infiltrating macrophages from resident microglia. In the chimeric mice, most of the infiltrating $\mathrm{GFP}^{+} \mathrm{Ibal}^{+}$cells in the site of the injury were arginase- 1 positive, suggesting that the infiltrating cells were highly skewed after injury. However, significantly fewer of the radio-resistant $\mathrm{GFP}^{-} \mathrm{Ibal}^{+}$microglia were arginase-1 positive, suggesting that macrophages infiltrating from the periphery are the primary source of alternatively activated myeloid cells (Fig. 7e, f). This preferential skew of infiltrating myeloid cells suggests that their phenotype switch took place in the periphery prior to infiltration rather than in the CNS parenchyma. These results in line with previous findings, suggesting that monocytes with an alternatively activated phenotype are arriving from a periphery through a unique path into the injured CNS (Shechter et al., 2013).

\section{Depletion of $T_{\text {reg }}$ exacerbates neurodegeneration after CNS injury}

The contribution of different subsets of $\mathrm{T}$ cells to neuronal survival after CNS injury has been intensively studied (Kipnis et al., 2002a; Ling et al., 2006; Lu et al., 2008; Moalem et al., 1999; Serpe et al., 1999), yet their role in this post-injury neuronal survival remains controversial (Jones et al., 2004; Reynolds et al., 2007; Zhao et al., 2012). Because $T_{\text {reg }}$ cells are known to exert asymmetric control of $T$ cell responses in non-pathological situations (Tian et al., 2011), we tested the hypothesis that $\mathrm{T}_{\text {reg }}$ cells were responding to injury in the draining lymph nodes, where they controlled the phenotype of $\mathrm{T}_{\text {eff }}$ cells. We used DEREG mice (Lahl et al., 2007), which express the diphtheria toxin receptor under the Foxp3 promoter to assess the effect of $\mathrm{T}_{\text {reg }}$ depletion on neuronal survival. Treatment of these mice with $40 \mu \mathrm{g} / \mathrm{kg}$ of diphtheria toxin (DTx) 2 
days before injury completely eliminates $T_{\text {reg }}$ cells in the bloodstream (Fig. S7a). Seven days after injury, the DEREG mice treated with DTx still displayed decreased numbers of $\mathrm{T}_{\text {reg }}$ cells in their CNS-draining deep cervical lymph nodes (Fig. 8a), and an increase in the number of activated $\mathrm{T}_{\text {eff }}$ cells in the deep cervical and skin-draining lymph nodes (Fig 8b, S7b).

To test the effect of $\mathrm{T}_{\text {reg }}$ cell depletion on CNS injury, we again used the optic nerve crush injury model. As expected from previous studies (Bettelli et al., 2006a), DEREG mice treated with DTx, and thus depleted of $\mathrm{T}_{\text {reg }}$ cells, showed a decrease in the number of surviving RGCs seven days after injury, as compared to wild type mice treated with DTx (Fig. 8c). We examined the contralateral retina of injured mice (Fig. S7c) and histological sections of uninjured mice treated with DTx (Fig. S7d), which did not display any loss of RGCs or immune cell infiltrate, suggesting DTx by itself did not have destructive effects on uninjured CNS tissue. Furthermore, there was no difference in neuronal survival in $\mathrm{C} 57 \mathrm{Bl} / 6$ mice treated with saline or DTx (Fig. S7e), confirming that DTx treatment was not causing non-specific effects at the dose we are using

Although no change in overall numbers of $\mathrm{CD}^{+} \mathrm{T}$ cells $\left(\right.$ Fig. 8d) or $\mathrm{CD} 11 \mathrm{~b}^{+}$ myeloid cells (Fig. 8e) at the site of injury was found, the phenotype of accumulated macrophages was altered in DEREG mice treated with DTx. A significant decrease in arginase-1 expressing CD68 (a marker of activated macrophages) cells was evident (Fig. 8f, g), suggesting a decrease in alternatively activated macrophages (M2-type) after injury in Treg-depleted mice. No difference in the total amount of $\mathrm{CD}^{+} 8^{+}$area was detected (Fig. S8a). To confirm the histological observations, we also examined the 
injured tissue by PCR. The mRNA expression of $\operatorname{argl}$, the gene for arginase-1, was reduced in DEREG mice, confirming the decrease in alternatively activated macrophages after $\mathrm{T}_{\text {reg }}$ depletion (Fig. 8h).

Exogenous $T_{\text {reg }}$ cells inhibit a beneficial response to CNS injury

A complete depletion of $\mathrm{T}_{\text {reg }}$ cells using DEREG mice resulting in impaired outcome of CNS injury in our optic nerve crush injury model (Fig. 8c). However, the question still remains whether increased activity of $T_{\text {reg }}$ cells would conversely offer a benefit after CNS injury. First, we tested the physiological outcome of $T_{\text {reg }}$ manipulation via potentiation of $\mathrm{T}_{\text {reg }}$ suppressive function by treating mice with all-trans retinoic acid (ATRA), which induces differentiation of $\mathrm{T}_{\text {reg }}$ cells (Mucida et al., 2007), stabilizes the $\mathrm{T}_{\text {reg }}$ phenotype (Zhou et al., 2010), and makes $\mathrm{T}_{\text {reg }}$ cells more suppressive (Zhou et al., 2010). As expected, treatment of mice with ATRA increased the $T_{\text {reg }}$ population in the deep cervical lymph nodes after injury (Fig. 9a) but surprisingly not in the SDLN (Fig. S9a) and resulted in a decrease of activated effector T cells in the deep cervical and skindraining lymph nodes (Fig. 9b, S9b). Interestingly, and in line with some reports (Kipnis et al., 2002a) but contrary to other previous findings (Kleinschnitz et al., 2013), mice treated with ATRA exhibited decreased neuronal survival compared to vehicle treated mice (Fig. 9c), suggesting that induction of highly suppressive $T_{\text {reg }}$ cells limits the protective $\mathrm{T}_{\text {eff }}$ responses. To rule out a possible in vivo effect of ATRA on cells other than $\mathrm{T}$ cells, we differentiated $\mathrm{T}_{\text {reg }}$ cells in vitro ( $\mathrm{iT}_{\mathrm{reg}}$ ) using ATRA and TGF $\beta$ (Fig. S9c). Injection of $\mathrm{iT}_{\text {reg }}$ cells into CNS-injured mice also resulted in an increase in $\mathrm{T}_{\text {reg }}$ cells (Fig. 9d) and attenuation of their activated $T_{\text {eff }}$ response to injury in the deep cervical 
lymph nodes (Fig. 9e), but no change in the number of $\mathrm{T}_{\text {reg }}$ cells and $\mathrm{T}_{\text {eff }}$ cells in the skindraining lymph nodes (data not shown) and a reduction in neuronal survival (Fig. 9f). In contrast, mice treated with $\mathrm{T}_{\text {eff }}$ cells (that were activated without TGF $\beta$ and ATRA, and that contained only $\sim 3 \%$ Foxp $^{+} \mathrm{T}_{\text {reg }}$ compared to $\sim 85 \%$ in $\mathrm{T}_{\text {reg }}$ designated culture conditions) did not show any change in neuronal survival (Fig. 9f), possibly due to the large number of $\mathrm{T}_{\text {eff }}$ cells already present in wild type mice.

$T_{\text {reg }}$ cells do not infiltrate the injured CNS after injury

Because $\mathrm{T}_{\text {reg }}$ cells are exerting a negative effect on the outcome to CNS injury, we sought to determine if $\mathrm{T}_{\text {reg }}$ cells were also gaining access to the site of injury. Upon injury, there is the influx of $\mathrm{T}_{\text {eff }}$ cells to the CNS parenchyma (Fig. 10a). Despite being able to visualize $T_{\text {reg }}$ cells using Foxp3 immunolabeling in the spleen (Fig. 10b) and in spinal cords that had been injected directly with $T_{\text {reg }}$ cells (Fig. 10c), we did not see $T_{\text {reg }}$ cells in the parenchyma of the injured optic nerve in animals after exogenous i.v. injection of $\mathrm{T}_{\text {reg }}$ cells (Fig. 10d) or in injured wild-type mice, wild-type mice injected with $\mathrm{T}_{\text {eff }}$ cells, DEREG mice treated with DTx, and wild-type mice treated with DTx (data not shown). Furthermore, there was no difference in the number of CD4 ${ }^{+} \mathrm{T}_{\text {eff }}$ cells

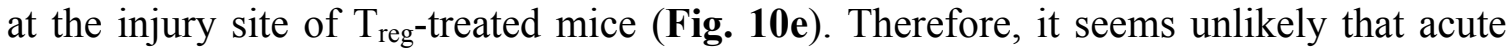
manipulation of $\mathrm{T}_{\text {reg }}$ cells in our experimental paradigms is affecting neuronal survival through the migration of effector T cells in to the site of the injury. Next, to determine if addition of $\mathrm{T}_{\text {reg }}$ cells could affect monocyte migration to the injured $\mathrm{CNS}$, we quantified the number of $\mathrm{CD} 1 \mathrm{~b}^{+}$cells accumulating at the site of injury. As with $\mathrm{CD}^{+} \mathrm{T}$ cells, there was no change in the number of $\mathrm{CD}_{1} 1 \mathrm{~b}^{+}$cells that migrated to the site of injury in 


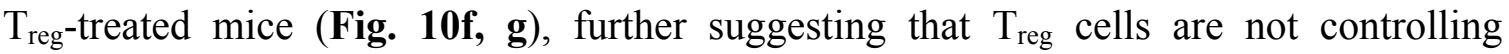
immune cell migration in this injury model.

To determine if $\mathrm{T}$ cell-derived cytokines are affected by $\mathrm{T}_{\text {reg }}$ manipulation after CNS injury, we examined the mRNA expression of genes from the optic nerve of injured mice treated with either $T_{\text {eff }}$ or $T_{\text {reg }}$ cells. Mice treated with $T_{\text {reg }}$ cells display a dramatic decrease in the amount of IL-4 mRNA compared to mice treated with $T_{\text {eff }}$ cells (Fig. 11a), suggesting a change in the Th2 response to damage at the injury site with $T_{\text {reg }}$ treatment. To determine if these changes are having effects downstream on myeloid cells after CNS injury, we examined markers of myeloid skewing in the injured optic nerves of mice treated with $\mathrm{T}_{\text {reg }}$ cells. Indeed, $\mathrm{T}_{\text {reg }}$ treated mice displayed a decrease in the mRNA expression of alternatively activated macrophage markers $\arg 1$ and $i l 10$, while there was no change in the classical activation markers nos 2 and $\operatorname{tnf}$ (Fig. 11b-e). To further demonstrate that there was a loss of alternative activation of macrophages in $\mathrm{T}_{\text {reg }}$ injected mice, we examined colocalization between CD68, a marker of activated myeloid cells, and argniase 1 by immunofluorescence. While $\mathrm{T}_{\text {eff }}$ injected mice displayed marked expression of arginase-1 in the $\mathrm{CD} 68^{+}$fraction 7 days after injury, injection of $\mathrm{T}_{\text {reg }}$ cells led to a decrease in arginase-1 expression by myeloid cells (Fig. 11f, g), further demonstrating that both addition and deletion of $\mathrm{T}_{\text {reg }}$ cells is detrimental in CNS trauma through their effects on the innate immune response at the site of injury. 


\section{Chapter IV: Discussion}

\section{Opening thoughts}

What makes the search for neuroprotective therapies after CNS injury especially pressing is that there currently are no treatments that have undergone rigorous testing in the clinical setting or approved by FDA for patient use. While several small phase I trials held promise for treatments such as hyperbaric oxygen treatment, progesterone treatment, and hypothermia, these treatments either have not yet undergone rigorous double-blind randomized trials, or have failed in these trials (Bennett et al., 2012; Ma et al., 2012; Sydenham et al., 2009).

While the pathways guiding the immune response to CNS injury have started coming to light, we are far from delivering a clinically useful therapeutic agent that can target the immune response after injury. The early efforts in the field were undertaken with the assumption that the immune response to injury had to be detrimental, due to the ability of the adaptive immune system to promote devastating autoimmunity in the CNS seen in diseases such as multiple sclerosis. This led to many studies and papers that explored the detrimental effects of immune cells on the CNS: production of neuro- and oligo-toxic TNF, the production of ROS, and infiltration of "inflammatory" cells such as neutrophils and monocytes. Because of this focus, the fact that the immune system could also promote benefit after injury did not become apparent until about 15 years ago (Moalem et al., 1999). Fortunately, the field of CNS trauma is finally starting to recognize that the response to this pathology needs to be understood at a much deeper 
level than the simplistic binary categorization of whether the immune response (either adaptive or innate) is beneficial or detrimental. Rather, we are moving toward an understanding that there are many factors that contribute to the final outcome from injury, including which cells respond, the intensity of the response, duration of the response, and whether this response is happening at the site or in a draining lymph node.

One of the chief problems with the targeting of the immune system so far has been that many of the therapies that have focused on the adaptive immune system have also induced an autoimmune pathology at the same time as providing neuroprotection (Moalem et al., 1999). It is this dual potential of the adaptive immune system is one of the most important puzzles that faces the field if indeed the adaptive immune system were to be targeted clinically: the immune system can promote both protection and destruction, but is it possible to promote protection while avoiding the detrimental effects of the immune system? Through my research, I have found two novel pathways by which that immune system-mediated neuroprotection can be boosted without inducing a detrimental autoimmunity. First, under experimental conditions, antigen specificity is not required for $\mathrm{T}$ cells to promote their neuroprotective ability, but rather signals such as IL$1 \beta$ derived from the injured tissue are alerting and shaping the immune response that consequently protects injured tissue from continuous degeneration. This suggests that a spontaneously induced autoimmune response that benefits injured tissue could be circumvented by antigen non-specific $\mathrm{T}$ cell response that could be boosted without a threat of autoimmune disease induction. 
Because CNS specific $\mathrm{T}$ cells are present in most healthy people, avoiding activation of antigen specific cells in the context of CNS injuries could prove to be clinically challenging. However, control of the regulatory $\mathrm{T}$ cells in patients is a more manageable goal. I have shown that $\mathrm{T}_{\text {reg }}$ cells differentially control both protective and destructive responses to CNS injury. Because of the differential regulation of the neuroprotective and neurodestructive phenotype of the $\mathrm{T}$ cells that respond, this regulation could then be targeted to elicit a protective response that doe not induce autoimmunity.

One of the common downstream mediators of both of these $\mathrm{T}$ cell manipulations is the production of T cell derived IL-4, which is able to promote neuroprotection through multiple mechanisms. We show two distinct mechanisms to induce IL-4 production by $\mathrm{CD}^{+} \mathrm{T}$ cells after injury. $\mathrm{T}_{\text {reg }}$ cells controlled the IL-4 expression of $\mathrm{CD} 4^{+} \mathrm{T}$ cells, and addition of exogenous $T_{\text {reg }}$ cells was able to suppress this endogenous production. Even more interesting is the ability of the injured CNS tissue to induce a Th2 skew in $\mathrm{CD}^{+} \mathrm{T}$ cells through MyD88 and IL-1 $\beta$ signaling and, which promoted a strong Th2 skew in $\mathrm{CD}^{+} \mathrm{T}$ cells that accumulate in the injured tissue.

Finally, we found that this adaptive-immune derived IL-4 can mediate neuroprotection through at least two different mechanisms in the injured CNS. Not surprisingly, given that it is well-known to promote this phenotype in vitro, IL-4 was able to induce an alternative activation of macrophages at the injury site. These macrophages then promote recovery through the production of matrix components and MMPs that are necessary to remodel tissue (Gordon and Martinez, 2010; Kigerl et al., 2009). More 
interesting, however, is that IL-4 is also acting directly on neurons through their IL-4R. This protective signaling is mediated through IRS1/2 adaptor molecules that signal downstream from IL-4R $\alpha$ (Blaeser et al., 2003) and potentiates endogenous neurotrophin signaling on neurons, leading to both increased survival and potentiated axonal outgrowth. Therefore, this signaling pathway is especially exciting, as it can act acutely to keep neurons alive and chronically to promote their functional recovery.

\section{Antigen non-specific effects of T cells in CNS injury}

One of my most unexpected findings is that antigen specificity is not required for $\mathrm{T}$ cells to exert their neuroprotective effects. $\mathrm{CD} 4^{+} \mathrm{T}$ cells canonically need to see their antigen in the context of MHCII in order to mediate their effects (Madsen et al., 1999), yet under defined experimental conditions $\mathrm{T}$ cells were able to exert beneficial effects on the injured CNS in the absence of antigen recognition signals. These results demonstrated that neuroprotection (studied after optic nerve crush injury), axon regrowth, and functional recovery (studied after spinal cord contusive injury), all benefit from an antigen-independent response of IL-4 producing T cells induced by damage associated mediators such as IL-1 $\beta$ that originate from the injured CNS. The injured tissue itself, then, induces a neuroprotective $\mathrm{T}$ cell response that is MyD88-dependent. Almost as surprising as the antigen-independence of the protective $\mathrm{CD}^{+} \mathrm{T}$ cell response is that these IL-4 producing T cells promote recovery after CNS injury directly via neuronal IL$4 \mathrm{R}$, potentiating neurotrophin signaling to promote neuronal survival and regrowth. While our work does not exclude the possibility that IL-4 can act through microglia or macrophages by promoting an alternative activation, this effect on neurons is especially 
interesting because in addition to survival, it also promotes functional regrowth of the injured tissue. With its ability to induce a protective response in both neurons and myeloid cells, IL-4 could be an excellent candidate for therapeutic intervention after CNS injury.

Our results demonstrate that once at the site of injury, a neuroprotective Th2 skew occurs independent of antigen recognition, presumably through damage associated mediators signaling directly on T cells in MyD88-dependent manner, which may underlie a rapid adaptive immune response to injury. Our results do not exclude the possibility that CNS self-antigen specific $\mathrm{T}$ cells exert a more potent neuroprotection than that of antigen non-specific cells, or that the endogenous $\mathrm{CD}^{+} \mathrm{T}$ cells that mediate neuroprotection in wild type mice can be boosted by vaccination with CNS self-antigen, as has been previously reported (Kipnis et al., 2002a). In fact, it could be that selfreactivity will potentiate the influx of CNS-reactive $\mathrm{T}$ cells regardless of their phenotype, and then these cells would become be skewed by signals from the injured tissue, becoming IL-4 producing and promoting a high degree of neuroprotection and recovery (Hendrix and Nitsch, 2007).

\section{The inflammasome product IL-1 $\beta$ : protective secondary alarmin after}

\section{CNS injury}

Just as the immune system is able to incorporate multiple stimuli to fashion a cohesive response, so too does the immune system require sensing of danger by multiple methods before it produces a strong response. While the alarmin(s) that initiate the response to CNS injury have yet to be elucidated, we show that the integrated sensing is 
done at the level of the NLRP3 inflammasome. This inflammasome then cleaves pro-IL$1 \beta$, which acts as a secondary alarmin, representing not the primary signal of tissue damage, but rather the integrated response of the resident cells to multiple danger signals. The activation of these inflammasomes, and thereafter IL-1 $\beta$ secretion, needs two stimuli in order to signal: one to upregulate the mRNA expression of the IL-1 $\beta$ gene and one to promote cleavage of the pro-cytokines into an active form which can then be released though a as-of-yet undefined secretory pathway to mediate its effects. Therefore, it is likely that ATP that is acutely released from the injured CNS (Davalos et al., 2005) is acting as the initial signal, as it needs to be sensed in order for microglia to migrate to the injury site and initiate the injury response. The identity of the second signal activating the response to injury has not yet been established.

Because IL-1 $\beta$ is not constitutively present, but rather upregulated after injury, it occupies a position that is different from the other alarmins. With its secretion after relevant stimuli and potentiation of the immune response through induction of other cytokines and chemokines, it act as an alarmin in every respect except that its expression is induced, albeit very rapidly (de Rivero Vaccari et al., 2008). In the CNS, IL-1 $\beta$ has been shown to promote activation of resident astrocytes and microglia (Proescholdt et al., 2002). However, we demonstrate here that is plays a much greater role than this limited activation of resident cells: while IL-1 $\beta$ has been largely viewed as a pro-inflammatory cytokine, it is able to promote both Th1 and Th2 T cell responses (Chung et al., 2009; Humphreys and Grencis, 2009). Indeed, in the context of injury, IL-1 $\beta$ acts not only as a rheostat, controlling the intensity of the immune response (Ben-Sasson et al., 2011; 
Schmitz et al., 2003), but is also a vital for helping to instruct the adaptive immune response about the proper, protective response.

Type I vs. Type II alarmins: different responses for different injuries?

Traditionally, alarmins have been thought to promote pro-inflammatory, type I immune responses including the production of pro-inflammatory cytokines and infiltration of cells that provide a respiratory burst and free radical production to clear bacterial and viral pathogens. However, with the elucidation of the alarmin IL-33, it is coming to light that alarmins can play a role in type II immune responses that are necessary for controlling extracellular pathogens through activation of both type 2 innate lymphoid cells (Neill et al., 2010) and Th2 T cells (Schmitz et al., 2005). In line with this type II immune response, IL-33 has been shown to play an important role in the immune defense against nematode infection (Humphreys et al., 2008), and to promote an allergic airway inflammation, both of which are characterized by a strong Th2 induction. Therefore, it seems that, as with the adaptive immune systems, alarmins are not a one size fits all solution to immune activation, but rather have specifically evolved to respond to different types of insults.

If alarmins can act as either a type I or type II immune stimulus, could the same then be true of IL-1 $1 \beta$ ? Despite its reputation as a pro-inflammatory cytokine, IL-1 $\beta$ also has the ability to promote $\mathrm{Th} 2$ responses in the proper context, such as in an allergic inflammation model (Schmitz et al., 2003) or, as we have shown, in that of the CNS after traumatic injury. IL-1 $\beta$ produced by microglia is playing a neuroprotective role in an optic nerve model CNS injury by inducing an antigen non-specific Th2 skew of myeloid cells that promote functional recovery. However, it is evident that IL-1 $\beta$ signaling in 
CNS injury promotes diverse reactions that can depend on the type of injury: depletion of IL-1 $\beta$ in bone marrow-derived cells leads to decreased survival in an optic nerve crush injury, but global deletion of IL-1 $\beta$ promotes benefit after spinal cord injury (Boato et al., 2013; Sato et al., 2012) This may have to do with the fact that spinal cord injuries induce a heavy neutrophils infiltration (Tjoa et al., 2003) that IL-1 $\beta$ potentiates, while optic nerve injury induces an inflammatory infiltration into the injury site that features a greater proportion of monocytes (personal observation, (Frank and Wolburg, 1996)), or may simply be due to the magnitude of the immune response that is elicited from each respective injuries. Indeed, it is clear that despite the advances that we have made, there is still much more to do to figure out how these systems are working.

\section{$T_{\text {reg }}$ cells in CNS injury}

Early work in models of stroke and Parkinson's disease showed that depletion of $\mathrm{T}_{\text {reg }}$ cells led to increased neurodegeneration, while increases in $T_{\text {reg }}$ cell numbers and function improved disease outcome (Liesz et al., 2009; Reynolds et al., 2007). More recent work, using the same manipulations, has shown that $\mathrm{T}_{\text {reg }}$ cells play a detrimental role after CNS injuries (Kleinschnitz et al., 2013), supporting the hypothesis that they are suppressing a beneficial autoimmune response (Kipnis et al., 2002a). There are several factors that may have contributed to these disparate findings. There are technical challenges with the current $T_{\text {reg }}$ depletion strategies that have hindered interpretation of depletion studies, such as targeting of activated effector cells with anti-CD25 treatment (Johnson et al., 2007a; Walsh and Kipnis, 2011). Furthermore, several studies have shown that $\mathrm{T}_{\text {reg }}$ cells have the potential to downregulate Foxp3 and become effector cells, 
especially when placed in lymphopenic or inflammatory conditions (Komatsu et al., 2009; Zhou et al., 2009a; Zhou et al., 2010), probably due to heterogeneity in the fate commitment of the $T_{\text {reg }}$ cell population (d'Hennezel et al., 2011), complicating transfer experiments into mice with abnormal adaptive immune systems. However, the conditions that drive this switch from $\mathrm{T}_{\text {reg }}$ to $\mathrm{T}_{\text {eff, }}$ and relevance of these models in vivo, is still a matter of debate (Rubtsov et al., 2010).

Our work further addresses the question of how $\mathrm{T}_{\text {reg }}$ cells are affecting the outcome from CNS injury. While we show that $T_{\text {reg }}$ cells have profound effects on neuronal survival from injury, they are not found at the site of the injury, but are rather enriched in the draining lymph node. $\mathrm{T}_{\text {reg }}$ cells are known to exert asymmetric control of $\mathrm{T}$ cell responses in non-pathological situations (Tian et al., 2011), raising the possibility that these $\mathrm{T}_{\mathrm{reg}}$ cells are exerting their action on the phenotype of $\mathrm{T}_{\text {eff }}$ cells in the draining lymph node. The $\mathrm{T}_{\text {eff }}$ cells, in turn, direct the phenotype of the infiltrating innate immune cells. Previous works demonstrated that precursors for alternatively activated macrophages arrive the injured CNS through a unique path of the choroid plexus (Shechter et al., 2013) and are coming "pre-determined" to differentiate into alternatively activated macrophages.

Several studies have shown that alternative activation of macrophages is a beneficial response to CNS injury (Fenn et al., 2014; Shechter et al., 2013) Tissue-building macrophages produce growth factors such as IGF1, VEGF, TGF $\beta$, and factors that remodel the extracellular matrix such as MMPs and RELM $\alpha$, and promote a tissue building phenotype in injured tissue (Gordon and Martinez, 2010). T cells, and 
specifically Th2 effector cells, produce several cytokines, such as IL-4 for example, that can induce alternatively activated macrophages (Van Dyken and Locksley, 2013). Our results suggest that $\mathrm{T}$ cells induced in the CNS-draining deep cervical lymph nodes control the phenotype of the infiltrating monocytes, and future studies need to concentrate on a better understanding of the molecular interactions between $\mathrm{T}$ cells and myeloid cells that results in myeloid cells of a particular phenotype to migrate to the site of injury.

Our results support the notion that a spontaneous immune response after CNS trauma is beneficial and is tightly regulated by $\mathrm{T}_{\text {reg }}$ cells (Kipnis et al., 2002a). Elimination of $\mathrm{T}_{\text {reg }}$ leads to an excessive immune response, which is detrimental for injured tissue. However, injection of $\mathrm{T}_{\text {reg }}$ cells or potentiation of their suppressive function inhibits a spontaneous immune response to injury and also results in impaired neuronal survival. Further works should be aimed at understanding the divergent properties of $\mathrm{T}_{\text {reg }}$ cells that lead to this dichotomous response to injury and finding the compounds that could alleviate $T_{\text {reg }}$ function yet preserve the beneficial nature/phenotype of $T_{\text {eff }}$ cells. Without better understanding of $T_{\text {eff }} / T_{\text {reg }}$ interactions after CNS injury, therapies for CNS injuries that primarily target the $T_{\text {reg }}$ compartment should be taken with extreme caution as alteration of $\mathrm{T}_{\text {reg }}$ may result in impaired outcome of CNS trauma.

\section{Final thoughts}

The response to CNS injury is a multi-faceted response that is designed to bring in the immune system and activate it in a manner that provides a degree of protection to the injured tissue. However, because there are only a limited number of stereotypical 
responses that can be called upon, the response to injury is not perfectly adapted to the stimulus, and can be modulated clinically to promote a better recovery (Moalem et al., 1999). Despite the great advances that have been made in discovering the mechanisms of immune involvement in CNS injuries, there is still great controversy in the field about almost every aspect of this pathology. This controversy stems partially from the complexity of the systems we are studying, but also from the variety of models that have been used to study sterile CNS injuries: open-skull fluid percussion, spinal cord contusion, spinal cord compression, closed-head brain contusion, cryo-injury, optic nerve injury, and stab wounds are just a few examples of models that are currently in use (Boato et al., 2013; Bush et al., 1999; Kipnis et al., 2004a; Kita et al., 1997; Semple et al., 2010a; Sewell et al., 2004; Shechter et al., 2009), making the difference that are all-toooften seen between studies difficult to interpret. While these works, despite their disparities, have built a good foundation towards developing immune-targeted therapeutics, much more light needs to be shed on how the immune system functions so that the neuroprotective responses to injury can be separated from the destructive effects that are the constant specter hiding behind immuno-therapy for these sterile injuries.

While there are still many unanswered questions, we have a much better understanding of the type of immune response that promotes recovery from injury and those that are promoting neurodegeneration. Furthermore, my contribution provided several new pathways within the immune response to injury that could be targeted to improve neuronal survival after CNS injury: IRS signaling in neurons, IL-4 signaling in myeloid cells, $T_{\text {reg }}$ control of the adaptive immune response in lymph nodes, and IL-1 $\beta$ 
production in macrophages that signals to $\mathrm{T}$ cells, just to name a few. While there are not yet clinical trials targeting these pathways, the pathways elucidated present many opportunities to intercede and ameliorate the disability that comes from this devastating pathology. 


\section{Chapter V: Materials and Methods}

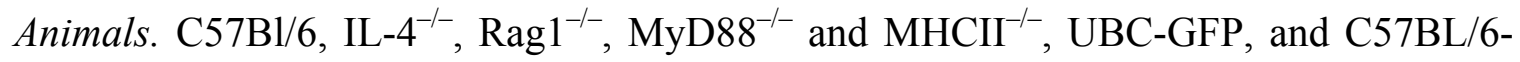
beta-actin-GFP strains of mice were purchased from Jackson Laboratories (Bar Harbor, $\mathrm{ME)}$; OTII/Rag $1^{-/}$mice were purchased from Taconic. KN2 mice were a gift from Dr. M. Mohrs (Trudeau Institute, NY). DEREG mice were a gift from Dr. T. Sparwasser (Institute of Infection Immunology, Twincore, Germany, (Lahl et al., 2007)). B10.PL and Balb/c-mice were purchased from Charles River, Sulzfeld Germany. IL-4-receptor ${ }^{--}$, IL$4 \mathrm{R}^{f l f f}:$ CamKII ${ }^{\text {cre }}$, and CamKII ${ }^{\text {cre }}$ mice were a kind gift by Monika Brunner-Weinzierl, DRFZ Berlin, David Wraith, Bristol and Talal Chatila, St. Louis, Gunther Schütz, DKFZ Heidelberg, respectively. IL-1 $\beta^{-/-}$(Shornick et al., 1996) and NLRP3 ${ }^{-/-}$(Kanneganti et al., 2006) mice are as previously described . All animals were housed in temperature and humidity controlled rooms, maintained on a $12 \mathrm{~h} / 12 \mathrm{~h}$ light/dark cycle (lights on 7:00 A.M.), and age-matched in each experiment. All strains were kept in identical housing conditions. All procedures complied with regulations of the Institutional Animal Care and Use Committee (ACUC) at The University of Virginia or with German guidelines on the use of laboratory animals.

Retrograde labeling of retinal ganglion cells. Mice were anesthetized, and the skull was exposed and immobilized in a stereotactic device. Holes are drilled in the skull above the superior colliculus (bilaterally $2.9 \mathrm{~mm}$ caudal to bregma and $0.5 \mathrm{~mm}$ lateral to midline). One $\mu \mathrm{L} 4 \%$ Fluoro-gold was injected $2 \mathrm{~mm}$ below the meningeal surface at a rate of 0.5 $\mu \mathrm{L} / \mathrm{min}$ using a Hamilton syringe and an automatic injector. The dye was allowed to 
diffuse into the tissue for 1 minute before the syringe was removed. The scalp was then sutured closed, and the mice allowed to recover on warming pads at $37^{\circ}$ before returning them to their home cages.

Optic nerve injury. Mice were subjected to an optic nerve injury three days after stereotactic surgery. Briefly, mice were anesthetized with a 1:1:8 mixture of ketamine:xylazine:saline. An incision was made in the connective tissue above the sclera. The venous sinus around the optic nerve was retracted to expose the optic nerve, and the nerve was crushed using an $\mathrm{N} 5$ self-closing forceps $2 \mathrm{~mm}$ behind the globe for three seconds. The mice were then allowed to recover at $37^{\circ}$ on a warming pad before returning to their home cages.

Retina excision. Mice were enucleated, and the cornea removed at the corenal limbus. The lens and the underlying vitreous were removed with forceps. The retina was separated from the sclera and pigment epithelium. Four cuts were made toward the optic disc, and the retina mounted on nitrocellulose paper and fixed in 4\% PFA overnight. Pictures of all four quadrants of the retina were taken at equal distances form the optic disc of the retinas using an Olympus IX-71 microscope. The pictures were then counted by a blinded observer to determine the number of RGCs per field.

Spinal cord injury. Mice were anesthetized with a 1:1:8 mixture of ketamine:xylazine:saline. A $15 \mathrm{~mm}$ mid-line skin incision was performed over the T6T13 vertebra, and the connective and muscle tissues were bluntly dissected to expose the lamina. A laminectomy was performed using rongeurs at $\mathrm{T} 9$ to expose the dorsal spinal cord. The vertebral column was stabilized with angled clamps attached to the T7 and T12 
transverse processes. A calibrated contusion injury of the spinal cord was induced by an Infinite Horizon Impactor (IH-0400 Impactor) or with a modified SPI Correx Tension/Compression Gage (Penn Tool, Maplewood, NJ, USA) at $10 \mathrm{cN}$ for 3 seconds. (Boato et al., 2013). After injury, the muscles and skin were sutured separately. Mice whose actual force varied by greater than $10 \%$ of the calibrated force were removed from analysis. The bladder of all mice were expressed twice a day, and were tested every two to three days for neurologic deficits using the Basso Mouse Scale for Locomotion (BMS) (Basso et al., 2006). BMS was performed by 2 independent investigators, both of whom were blind to the group identity.

Injection of labeled T cells after SCI . After MACS separation, T cells were washed twice with PBS were injected into the spinal cord lesion ( $1 \times 10^{5}$ cells in $1 \mu 1$ per mouse) using a Hamilton micropipette. For tracing T-cell migration in the spinal cord, they were incubated with $5 \mu \mathrm{M}$ CFDA-SE (Molecular Probes, C1157) in PBS ( $1 \times 10^{7}$ cells $\left./ \mathrm{ml}\right)$ for 5 min.

$T$ cell adoptive transfer. Total lymphocytes were isolated from total lymph nodes of naïve mice. The lymph nodes were passed through a $70 \mu \mathrm{m}$ screen to obtain a single-cell suspension. The $\mathrm{CD}^{+} \mathrm{T}$ cell population was negatively enriched on autoMACS using the $\mathrm{CD}^{+} \mathrm{T}$ cell isolation kit (Miltenyi) according to the manufacturer's instructions, and $3 \times 10^{6} \mathrm{CD}^{+} \mathrm{T}$ cells were injected i.v. into $\mathrm{RAG}^{-/-}$recipients. The $\mathrm{T}$ cells were allowed to reconstitute for the indicated times before performing optic nerve injury. Engraftment was confirmed by flow cytometry. 
Bone marrow chimeras. C57B1/6 mice were subjected to split dose irradiation, receiving a $350 \mathrm{rad}$ dose to sensitize the mouse followed 24 hours later by a lethal $950 \mathrm{rad}$ dose. Two hours after the second dose of irradiation, the mice were injected i.v. with $10^{7}$ bone marrow cells derived from the femur and tibia of donor mice, as previously described. For head covered irradiation, heads were covered with lead shields during both doses of irradiation. Briefly, femurs and tibia were removed from mice and muscles and tendons were cleaned from the bones. Both ends were cut off, and the marrow was flushed out using a 26 gauge needle with PBS containing 1\% FBS. Red blood cells were lysed using ACK lysis buffer, and live cells were counted and resuspended at $10^{8}$ cells per mL. The bone marrow was allowed to engraft at least 4 weeks before the mice were used for experiments.

Thymus implantation. This procedure was performed as previously described (Buch et al., 2006). Briefly, the thymus was removed from P5 donor pups. Three week old mice were anesthetized with a 1:1:8 mixture of ketamine:xylazine:saline. A $15 \mathrm{~mm}$ incision was made on skin and peritoneum at the right flank of the recipient mouse, and the kidney was exposed. A scalpel was used to scratch the kidney capsule, and the thymus was implanted below the capsule. The kidney was then returned to the peritoneum, and the peritoneum and skin were sutured closed. The mice were allowed to recover at $37^{\circ}$ on a heating pad before returning to their cages. T cell engraftment was allowed to take place for at least 6 weeks prior to any further manipulation.

Deep cervical lymph node removal. Mice were anesthetized with a 1:1:8 mixture of ketamine:xylazine:saline. A $10 \mathrm{~mm}$ incision was made midline above the trachea. 
Salivary glands and sternocleidomastoid muscles were retracted bilaterally to expose the deep cervical lymph nodes. Deep cervical lymph nodes were removed using Dumont forceps, and the skin was sutured closed. Mice that received sham surgery had their deep cervical lymph nodes exposed, and then the skin was sutured. The mice were allowed to recover on a $37^{0} \mathrm{C}$ warming pad before returning to their cages. Mice were allowed to recover from surgery for at least two weeks before optic nerve injury.

In vivo drug treatment. $200 \mu \mathrm{g}$ all-trans retinoic acid (Fisher) was dissolved in corn oil and injected i.p. every other day starting 3 days before injury. Diphtheria toxin was dissolved at $40 \mu \mathrm{g} / \mathrm{kg}$ in PBS and was injected into C57B1/6 or DEREG mice two days before optic nerve injury and on the day of optic nerve injury.

In vivo brefeldin A treatment. Two weeks post spinal cord injury, mice were injected i.v. with $300 \mu \mathrm{g}$ of brefeldin A. Five hours post-injection, mice were sacrificed, and spinal cords were dissociated with papain and prepared for flow cytomtery as described below.

Flow cytometry. For flow cytometric analysis of lymph nodes, the lymph nodes were isolated and passed through a $70 \mu \mathrm{m}$ strainer in PBS containing 1\% BSA and $2 \mathrm{mM}$ EDTA to obtain a single cell suspension. For flow cytometric analysis of spinal cords, the spinal cords were removed from mice, and the spinal meninges were removed from spinal cords. The spinal cords were minced, and then were dissociated for 45 minutes at $37^{\circ}$ in $4 \mathrm{U} / \mathrm{mL}$ papain (Worthington Biochemical) with $0.004 \%$ DNase added (Sigma Aldrich). The spinal cords were then triturated to form a single-cell suspension. The cells were spun down and resuspended in $50 \mu \mathrm{L}$ of unlabeled anti-CD16/32 in FACS buffer for 20 minutes at $4^{\circ}$, then $50 \mu \mathrm{L}$ of antibody staining cocktail was added for 30 minutes at 
$4^{\circ}$, after which the cells were washed and analyzed. The following antibodies were used, and are all from eBioscience unless otherwise noted: B220-PE, CD4-PerCp Cy5.5, CD8eFluor 450, CD19-PE, CD25-PE (BD Bioscience), CD45-APC, CD69-PE Cy7, Foxp3Alexa 488, hCD2-APC, IFN $\gamma$-APC, IL-4-PE, and TCR $\beta$-APC eFluor780. For intranuclear staining, the cells were fixed overnight in Foxp3 Fix/Perm buffer (eBioscience) before incubating with Foxp3 antibody in FACS buffer containing 0.3\% saponin (Fisher).. The samples were run on cytometers at the UVA Flow Core.

$T$ cell skewing assay. Total lymph nodes were removed from mice, and a single cell suspension was made by passing the cells through a $70 \mu \mathrm{m}$ strainer. $\mathrm{CD}^{+}$and $\mathrm{CD}^{-}$ lymphocyte fractions were isolated on autoMACS using magnetic bead separation (Miltenyi) and incubated in a 2:1 ratio $\left(2 \times 10^{6} \mathrm{CD}^{-} / \mathrm{mL}: 1 \times 10^{6} \mathrm{CD}^{+} / \mathrm{mL}\right)$ in the presence or absence of 3 optic nerves $/ \mathrm{mL}$ in $\mathrm{T}$ cell culture media consisting of RPMI supplemented with $10 \%$ FCS, $10 \mathrm{mM}$ HEPES, NEAA, Na-pyruvate, $50 \mathrm{mM} 2$ mercaptoethanol, L-glutamine, and pen-strep (Invitrogen). Optic nerves were obtained from either naïve uninjured or from mice injured three days prior to excision. It is clearly stated in the text for each experiment whether the optic nerve was obtained from injured or uninjured donors. In transwell experiments, nerves were put on top of a $0.4 \mu \mathrm{m}$ transwell insert, and in the $\mathrm{T}$ cell only experiments the $\mathrm{CD} 4^{-}$fraction was omitted. After 72 hours of culture, $\mathrm{CD}^{+} \mathrm{T}$ cells were re-isolated as described above, and RNA was extracted for qRT-PCR. For in vitro intracellular staining of $\mathrm{CD}^{+} \mathrm{T}$ cells, cells were cultured with $1 \mu \mathrm{g}$ plate-bound anti-CD3 and $1 \mu \mathrm{g}$ soluble anti-CD28 for 5 days. The cells were then stimulated with PMA/ionomycin for 5 hours, the last 4 of which $3 \mu \mathrm{g} / \mathrm{mL}$ 
of brefeldin a is added. Cells were then stained for extraceullular markers, fixed in IC fixation buffer (eBioscience), and stained for intracellular markers in $0.3 \%$ soponin.

Primary neuronal cultures. Primary neuronal cells were prepared from embryonic day 15 (E15) BALB/c mouse cortices by enzymatic dissociation using $0.5 \%$ trypsin and DNase treatment $(0.1 \mathrm{mg} / \mathrm{ml})$ in Hank's balanced salt solution (HBSS), followed by mechanical dissociation by trituration to obtain a single cell suspension. Cells were seeded on polyD-lysine coated cell culture plates directly (96-well plate) or on coated coverslips placed inside the wells of a $24-w e l l$ plate, and cultured at $37^{\circ} \mathrm{C}$ and $5 \% \mathrm{CO} 2$ in Neurobasal medium containing $2 \% \mathrm{~B}-27,1 \% \mathrm{~L}$-glutamine and $1 \%$ penicillin/streptomycin for $2 \mathrm{~h}$ prior to the start of the experiments. All cell culture reagents were from Gibco ${ }^{\circledR}$ (Invitrogen, Germany). Neurite length from single neuronal cells was analyzed using ImageJ analysis software (NIH) as described (Slaets et al., 2014).The length of the longest neurite of 50 single neurons per condition was measured using the following criteria: Neurites innervating other neurons and neurites running out of the picture were excluded from the data, and the length should be longer than the diameter of the cell body. The mean neurite length per condition was determined and data were expressed as mean \pm s.e.m. Experiments were repeated four times $(n=4)$.

Western blot. Cells were grown for six days in neuronal medium, IL-4 (10 ng/ml) was added $2 \mathrm{~h}$ before the addition of NT-3 (10 ng/ml, Peprotech Inc, Rocky Hill, NJ) for 5 min. Cells were lysed and protein concentrations were determined by the BCA protein assay (Pierce, Rockford, IL). Ten $\mu \mathrm{g}$ of each sample were electrophoresed on $10 \%$ SDSPAGE and electrotransferred to PVDF-membranes, which were blocked with $2 \%$ BSA 
in TBST for $1 \mathrm{~h}$ at room temperature. Primary anti phospho-MAPK p42/44 (1:250, Cell Signaling, Beverly, MA) were incubated overnight at $4{ }^{\circ} \mathrm{C}$. The HRP-linked secondary antibodies were incubated for $1 \mathrm{~h}$ at room temperature (1:5000) and signals detected using the ECL Plus System (all Amersham Pharmacia, Piscataway, NJ). Membranes were stripped and Western Blotting was performed as described above with primary antibodies of the non-phosphorylated protein forms (1:250, Cell Signaling, Beverly, MA) and HRPlinked anti-rabbit antibody (1:5000). Densitometric analysis was performed with Alpha Imager software. Values were calculated as percentage of phosphorylated protein to total protein. For IL-4 signaling the values were normalized to control (= $100 \%$ ), for the IL-4/ NT-3 interaction the values were normalized to NT-3 $(=100 \%)$.

qRT-PCR. RNA was isolated with the Bioline Isolate RNA kit, according to the manufacturer's instructions. cDNA was synthesized using the Applied Biosystems High Capacity Reverse Transcription Kit according to the manufacturer's instructions, and the resulting cDNA was analyzed on a CFX384 qPCR system from Bio-Rad using a Taqman primer for Gata3, with Gapdh as an internal loading control (Applied Biosystems).

Generation of concanavalin A (ConA)-activated T cells. Briefly, we prepared cultures of mixed lymphocytes from spleen and lymph nodes $\left(4 \times 10^{6}\right.$ cells $\left./ \mathrm{ml}\right)$ and stimulated with 1 $\mu \mathrm{g} / \mathrm{ml}$ ConA. To generate Th1 T cells, we added $1 / 50 \mathrm{ng} / \mathrm{ml}$ recombinant mouse IL-12 (R \& D Systems, Abingdon, UK) and $1 \mu \mathrm{g} / \mathrm{ml}$ anti-mouse IL-4 (clone 11B11, BD Pharmingen, Heidelberg, Germany). To generate Th2 cells, $4 \mathrm{ng} / \mathrm{ml}$ recombinant mouse IL-4 (BD Pharmingen, Heidelberg, Germany) and $1 \mu \mathrm{g} / \mathrm{ml}$ anti-mouse IL-12 (BD Pharmingen, Heidelberg, Germany) were added. Between days 7-9, the $T$ cells were 
restimulated with ConA, using irradiated splenocytes and thymocytes functioning as antigen-presenting cells. This was carried out in the presence of IL-12 and anti-IL-4 for the expansion of Th1 cells or IL-4 and anti-IL-12 for the expansion of Th2 cells. After restimulation, $\mathrm{CD}^{+} \mathrm{T}$ cells were prepared by MACS (Miltenyi Biotec, BergischGladbach, Germany).

Co-cultures of organotypic cortex explants and Thelper cells, and EC with hippocampal slices Briefly, collagen type I from rat tail (Sigma-Aldrich, Taufkirchen, Germany) was dissolved in $0.1 \mathrm{M}$ acetic acid at a final concentration of $2 \mathrm{mg} / \mathrm{ml}$. Of the collagen solution, $1 \mathrm{ml}$ was mixed with $50 \mu \mathrm{l}$ DMEM medium (Gibco, Karlsruhe, Germany) and neutralized ( $\mathrm{pH} 7.4)$ with reconstitution buffer $(2.2 \%$ sodium bicarbonate in $0.8 \mathrm{M} \mathrm{NaOH}$ solution). Organotypic slice cultures were prepared as follows. Briefly, the entorhinal cortex was dissected from P2 murine brains and cut into $350 \mu \mathrm{m}$-thick slices using a tissue chopper (Technical Products International, St. Louis, MO, USA). Collagen drops (30 $\mu$ l) containing homogenously distributed $\mathrm{T}$ cells were placed on a glass slide and each slice was placed directly next to the explants derived from mice of the same strain in a collagen matrix $\left(1.2 \times 10^{5}\right.$ cells/drop, 1 drop $=30 \mu 1$ collagen $)$. Consecutive covering of the $\mathrm{T}$ cell drop and the explant with another drop of collagen $(30 \mu \mathrm{l})$ ensured a standardized distance from the edge of the $\mathrm{T}$ cell drop. The sterile cultivation medium contained 25\% HBSS, 25\% heat-inactivated normal horse serum, 8 g/L MEM Hepes, 4 $\mathrm{mM}$ L-glutamine, $4 \mathrm{mg} / \mathrm{L}$ insulin, $0.58 \%$ bicarbonate solution (Gibco, Karlsruhe, Germany), 1.2\% Glucose-20 (Braun, Melsungen, Germany), 1\% penicillin-streptomycin solution (100x), $0.8 \mathrm{mg} / \mathrm{L}$ vitamin $\mathrm{C}$ and $5 \mathrm{mM}$ Trisbase (Sigma-Aldrich, Taufkirchen, Germany) at $\mathrm{pH} 7.35$. The collagen co-cultures were incubated at $37{ }^{\circ} \mathrm{C}$ in a humidified 
atmosphere with $5 \% \mathrm{CO}_{2}$. After $48 \mathrm{~h}$ in vitro, the collagen co-cultures were analyzed microscopically. Co-cultures of EC and hippocampal slices derived from beta-actin-GFP mice (EC) and wt mice (hippocampus) were obtained and prepared as described elsewhere (Hechler et al., 2010). Recombinant IL-4 was directly applied to the culture medium.

Measurement of axonal growth from cortical slices. To evaluate axonal outgrowth from the explants, we employed a highly reliable evaluation procedure described previously, using image analysis software (Image J, Wayne Rasband, NIH) to quantify axonal density after 2 days in culture. Neurite outgrowth was photo-documented at a total magnification of 100, using a 10x Olympus LCPLANFL objective (Olympus IX70, Hamburg, Germany). To determine axonal density, image processing was based on the Sobel algorithm, which performs a 2-D spatial gradient measurement in a microphotograph and so emphasizes regions of high spatial density that correspond to axons in the area investigated. To determine the axonal density the mean intensity was calculated in a standardized area in a microphotograph of every single cortical slice.

Inhibitors. The following blocking antibodies were mixed into the collagen and compared to the corresponding control antibodies in the same concentration: rabbit anti-mouse NGF (2 $\mu \mathrm{g} / \mathrm{ml}$, IBT, Reutlingen, Germany), rabbit anti-NT4 (25 $\mu \mathrm{g} / \mathrm{ml}$, Millipore, Schwalbach/Ts, Germany), chicken anti-human NT-3 (25 $\mu \mathrm{g} / \mathrm{ml}$, Promega, Madison, WI, USA), chicken IgY (25 $\mu \mathrm{g} / \mathrm{ml}$, Promega, Madison, WI, USA), rabbit IgG (27 $\mu \mathrm{g} / \mathrm{ml}$, Sigma, Taufkirchen, Germany). 
Image analysis of EC-hippocampal co-cultures. For the analysis of $\mathrm{EGFP}^{+}$axons in the EGFP/wildtype co-culture model, the average intensity in a standardized area $(<1 \%$ deviation in area size) was compared using MetaMorph Image Software (Visitron Systems, Munich, Germany).

Immunofluorescent staining of CST tissue. Immunofluorescent stainings were performed using standard protocols. Briefely, after administrating an overdose of anesthetics, mice were transcardially perfused with $4 \%$ PFA. The spinal cord was prepared, postfixed for 1 hour in 4\% PFA, cut on a vibratome and antigen retrieval (EnVision Flex, DAKO, Glostrup, Denmark) was performed using standard procedures. Spinal cord slices were subsequently treated with $5 \%$ NGS and $0,1 \%$ Triton for 1 hour and were incubated with monoclonal antibody against the IL4 receptor alpha chain (BD Biosciences, Heidelberg, Germany) for $48 \mathrm{~h}$ at $4^{\circ} \mathrm{C}$ which was visualized by a secondary AL Fluor 488 labeled antibody (Invitrogen, Life Technologies GmbH, Darmstadt, Germany). Slices were subsequently incubated with a monoclonal antibody against SMI 312 (Covance, Princeton, NJ, USA) for 3 hours at room temperature and visualized by a secondary AL Fluor 568 labeled antibody. Images were taken using a Leica SP8 confocal microscope (Leica, Wetzlar, Germany). To test for antibody specificity, wild-type and IL4- $\mathrm{R}^{-/-}$spinal cord slices were imaged using the same microscope settings.

Immunohistochemical staining of optic nerve tissue. For arginase-1 staining, mice were perfused transcardially with ice-cold PBS containing $4 \mathrm{U} / \mathrm{mL}$ heparin, then with $4 \%$ paraformaldehyde. Eyes were enucleated, and frozen on dry ice in OCT. Ten $\mu \mathrm{m}$ sections were cut on a Lyca cryostat and mounted on gelatin coated slides. Sections were then 
stained for arginase-1 (Santa Cruz Biotechnology, clone V20), CD68 (Biolegend, clone FA11), Iba1 (Biocare Medical, polyclonal) and GFP (Abcam, polyclonal). For CD4 and CD11b staining, mice were perfused transcardially with ice-cold PBS containing $4 \mathrm{U} / \mathrm{mL}$ heparin. Eyes were enucleated and frozen on dry ice in OCT. Ten $\mu \mathrm{m}$ sections were cut on a Lyca cryostat and mounted on gelatin coated slides. Slides were post-fixed in 3:1 acetone:ethanol at 4 degrees before staining with the flowing antibodies: CD4-FITC (eBioscience, clone GK-1.5), CD11b (Biolegend, clone M1/70), Foxp3-biotin (eBioscience, clone FKJ-16s). For Foxp3 and CD4 co-staining, CD4 was detected with an anti-fluorescein secondary antibody (Life Technologies) and Foxp3 was detected with Alexfluor 594 conjugated streptavidin (Jackson Immunochemical)

$T$ cell cultures. For $\mathrm{T}_{\text {eff }}$ cultures, total lymph nodes were dissected, and a single cell suspension made by mashing through a $70 \mu \mathrm{m}$ mesh. $3 \times 10^{6}$ cells $/ \mathrm{mL}$ were incubated in $\mathrm{T}$ cell culture media supplemented with $1 \mu \mathrm{g} / \mathrm{mL}$ anti-CD3 (clone 145-2C11, ATCC stock \# CRL-1975, antibody grown and isolated by UVA lymphocyte culture center), and 1 $\mu \mathrm{g} / \mathrm{mL}$ anti-CD28 (clone 37.51, Bioxcell, Stock \#BE0015-1). For $\mathrm{iT}_{\text {reg }}$ cultures, the media was supplemented with $10 \mathrm{nM}$ ATRA (Fisher), $5 \mathrm{ng} / \mathrm{mL}$ TGF $\beta$ (Peprotech), and $250 \mathrm{U} / \mathrm{mL}$ IL-2 (R\&D). The cultures were maintained for 5 days before $\mathrm{CD}^{+} \mathrm{T}$ cells were isolated using magnetic bead separation (Miltenyi) and injected i.v. into C57Bl/6J mice.

Macrophage skewing assay. Bone marrow was isolated from WT mice and cultured on untreated petri dishes in DMEM/F12 containing $10 \mathrm{ng} / \mathrm{mL}$ MCSF (eBioscience), $10 \%$ FCS, L-glutamine, and pen-strep (Invitrogen). The media was changed every 3 days, and 
macrophages were used after 8 days in vitro. The day before the macrophages were used, they were re-plated on TC-treated 24 well plates. $\mathrm{CD}^{+} \mathrm{T}$ cells from injured or uninjured deep cervical or skin-draining lymph nodes were isolated using magnetic bead separation (Miltenyi) and incubated at $1 \times 10^{6}$ cells/well in complete macrophage media. 24 hours after addition of $\mathrm{T}$ cells, the macrophages were washed $5 \mathrm{x}$ with PBS to remove the nonadherent T cells, and RNA was isolated from the macrophages. 


\section{Chapter VI: Figures and Legends}

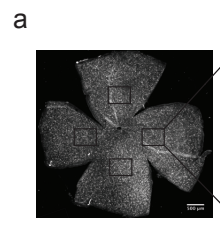

b

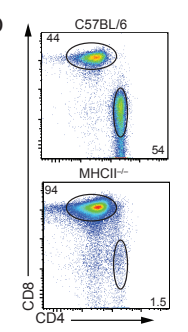

f
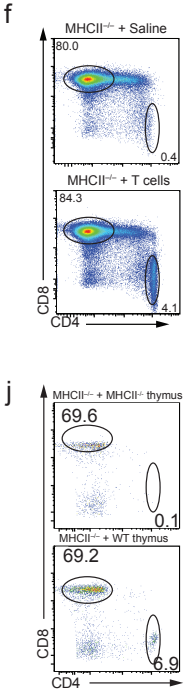

c

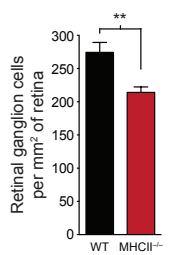

g
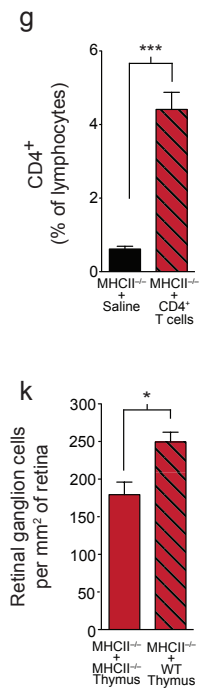

WT Injured

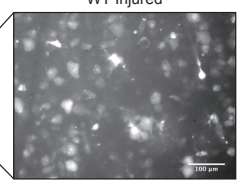

d

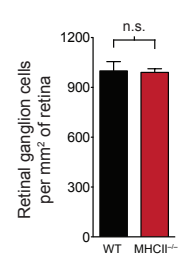

h
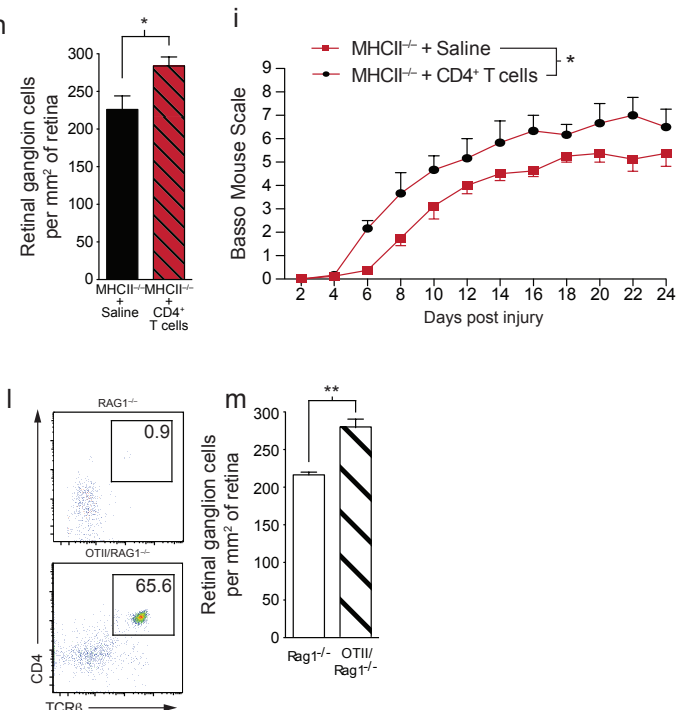

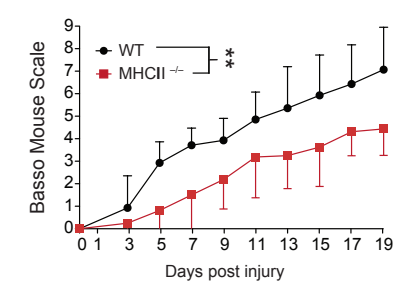

MHCII+- Injured

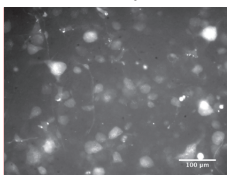

e

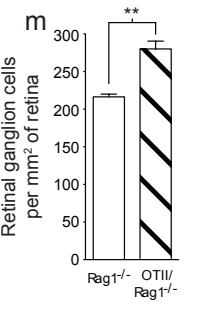

Fig. 1. MHCII-independent induction of neuroprotective $\mathrm{CD}^{+} \mathrm{T}$ cells in response to CNS injury. (a) Representative images from wild type or $\mathrm{MHCII}^{-/-}$retinas labeled with the retrograde tracer Fluoro-Gold. Images are from retinas 7 days post-injury (scale bar $=$ $500 \mu \mathrm{m}$ (retina whole mount) or $100 \mu \mathrm{m}$ (individual field)). (b) $\mathrm{MHCII}^{-/-}$mice have few $\mathrm{CD}^{+} \mathrm{T}$ cells. Representative flow cytometry plots of $\mathrm{CD} 4^{+}$and $\mathrm{CD} 8^{+}$lymphocytes in the 
deep cervical lymph nodes of $\mathrm{C} 57 \mathrm{Bl} / 6$ or $\mathrm{MHCII}^{-/-}$mice. Numbers indicate $\mathrm{CD}^{+}$cells (bottom right) and $\mathrm{CD} 8^{+}$cells (top left) as a percent of $\mathrm{TCR} \beta^{+}$cells. (c) $\mathrm{MHCII}^{--}$mice have impaired neuronal survival. Retinal ganglion cell survival (mean \pm s.e.m.) after optic nerve crush injury in $\mathrm{MHCII}^{-/}$or wild type mice, assessed 7 days after injury by Fluoro-Gold staining ( $\mathrm{n}=8$, wild type; $\mathrm{n}=11, \mathrm{MHCII}^{--} ; * *, \mathrm{p}<0.01$; Student's t-test, representative of two experiments). (d) $\mathrm{MHCII}^{--}$and wild type counterparts show no difference in the retinal ganglion cell density in their uninjured retinas. Retinal ganglion cell counts (mean \pm s.e.m.) in the contralateral retina of $\mathrm{MHCII}^{-/-}$or wild type mice, assessed 7 days after injury by Fluoro-Gold staining $\left(\mathrm{n}=8\right.$, wild type; $\mathrm{n}=11, \mathrm{MHCII}^{-/-}$, Student's t-test, representative of two experiments). (e) Poor functional recovery of $\mathrm{MHCII}^{-/}$mice after spinal cord injury. Locomotor score (mean \pm s.e.m.) of C57Bl/6 or $\mathrm{MHCII}^{-/}$mice after a $70 \mathrm{kDy}$ impact below the T9-T10 vertebra was assessed by a blinded observer using the Basso Mouse Scale ( $\mathrm{n}=7, \mathrm{C} 57 \mathrm{Bl} / 6 ; \mathrm{n}=8, \mathrm{MHCII}^{-/} ;$*, $\mathrm{p}<$ 0.05; Repeated-measure two-way ANOVA with Bonferroni's post-test, representative of two experiments). (f, $\mathbf{g}$ ) Injection of naïve $\mathrm{CD}^{+} \mathrm{T}$ cells into $\mathrm{MHCII}^{--}$mice, partially restores their population of $\mathrm{CD}^{+} \mathrm{T}$ cells. Representative flow cytometry plots (f) and quantification (g) of $\mathrm{CD} 4^{+}$and $\mathrm{CD} 8^{+}$lymphocytes in the deep cervical lymph nodes of $\mathrm{MHCII}^{-/-}$mice or $\mathrm{MHCII}^{-/-}$mice injected with $3 \times 10^{6} \mathrm{CD}^{+} \mathrm{T}$ cells. Numbers indicate $\mathrm{CD} 4^{+}$cells (bottom right) and $\mathrm{CD} 8^{+}$cells (top left) as a percent of $\operatorname{TCR} \beta^{+}$cells $(\mathrm{n}=5$, $\mathrm{MHCII}^{--} ; \mathrm{n}=6, \mathrm{MHCII}^{--}+\mathrm{CD}^{+} \mathrm{T}$ cells; ${ }^{* * *}, \mathrm{p}<0.001$; Student's t-test, representative of two experiments). (h) Injection of naïve $\mathrm{CD}^{+} \mathrm{T}$ cells into $\mathrm{MHCII}^{-/-}$mice increases neuronal survival. Retinal ganglion cell survival (mean \pm s.e.m.) after optic nerve crush injury in $\mathrm{MHCII}^{--}$mice or $\mathrm{MHCII}^{-/-}$mice injected with $3 \times 10^{6}$ naïve $\mathrm{CD}^{+} \mathrm{T}$ cells on 
the day of injury, assessed 7 days after injury by Fluoro-Gold staining $\left(\mathrm{n}=5, \mathrm{MHCII}^{--} ; \mathrm{n}\right.$ $=6, \mathrm{MHCII}^{--}+\mathrm{CD}^{+} \mathrm{T}$ cells; $*, \mathrm{p}<0.05$; Student's t-test, representative of two experiments). (i) Improved functional recovery of $\mathrm{MHCII}^{-/-}$mice that receive naïve $\mathrm{CD} 4{ }^{+}$ $\mathrm{T}$ cells after spinal cord injury. Locomotor score (mean \pm s.e.m.) after injury of $\mathrm{MHCII}^{-/}$ mice or $\mathrm{MHCII}^{--}$mice injected with $3 \times 10^{6} \mathrm{CD}^{+} \mathrm{T}$ cells the day after injury and 8 days after injury was assessed by a blinded observer using the Basso Mouse Scale ( $\mathrm{n}=4$ mice per group; *, $\mathrm{p}<0.05$; Repeated-measure two-way ANOVA with Bonferroni's post-test; representative of two experiments). (j) $\mathrm{MHCII}^{-/-}$mice implanted with wild type thymi have increased $\mathrm{CD} 4^{+} \mathrm{T}$ cells. Representative flow cytometry plots of $\mathrm{CD} 4^{+}$and $\mathrm{CD} 8^{+}$ lymphocytes in the deep cervical lymph nodes of $\mathrm{MHCII}^{-/-}$mice implanted with either $\mathrm{MHCII}^{--}$or wild type thymi. Numbers indicate $\mathrm{CD} 4^{+}$cells (bottom right) and $\mathrm{CD} 8^{+}$cells (top left) as a percent of TCR $\beta^{+}$cells. (k) $\mathrm{MHCII}^{-/-}$mice implanted with wild type thymi display improved neuronal survival over those implanted with a $\mathrm{MHCII}^{--}$thymus. Retinal ganglion cell survival (mean \pm s.e.m.) 7 days after injury of $\mathrm{MHCII}^{-/-}$mice implanted with either $\mathrm{MHCII}^{--}$or wild type thymi (six weeks after implantation) as assessed by Fluoro-Gold staining ( $\mathrm{n}=5$, wild type thymus; $\mathrm{n}=8 \mathrm{MHCII}^{-/-}$thymus; *, $\mathrm{p}<$ 0.05; Student's t-test; representative of two experiments). (I) OTII/Rag $1^{-/-}$mice develop $\mathrm{CD}^{+} \mathrm{T}$ cells, while Rag ${ }^{-/}$mice do not. Representative flow cytometry plots of $\mathrm{CD} 4^{+}$ lymphocytes in the deep cervical lymph nodes of $\operatorname{Rag} 1^{-/-}$mice or OTII $/ \operatorname{Rag} 1^{-/-}$mice. Numbers indicate $\mathrm{CD} 4^{+} \mathrm{TCR} \beta^{+}$cells as a percent of $\mathrm{CD} 45^{+}$cells. (m) OTII/Rag $1^{-/-}$mice have improved neuronal survival from Rag $1^{-/}$mice. Retinal ganglion cell survival (mean \pm s.e.m.) of Rag $1^{-/-}$and OTII/Rag $1^{-/-}$mice assessed 7 days after injury by Fluoro-Gold 
staining $\left(\mathrm{n}=6, \operatorname{Rag}^{-/-}\right.$and $\mathrm{n}=10, \mathrm{OTII} / \operatorname{Rag} 1^{-/-} ; * *, \mathrm{p}<0.01$; Student's t-test; representative of three experiments). 

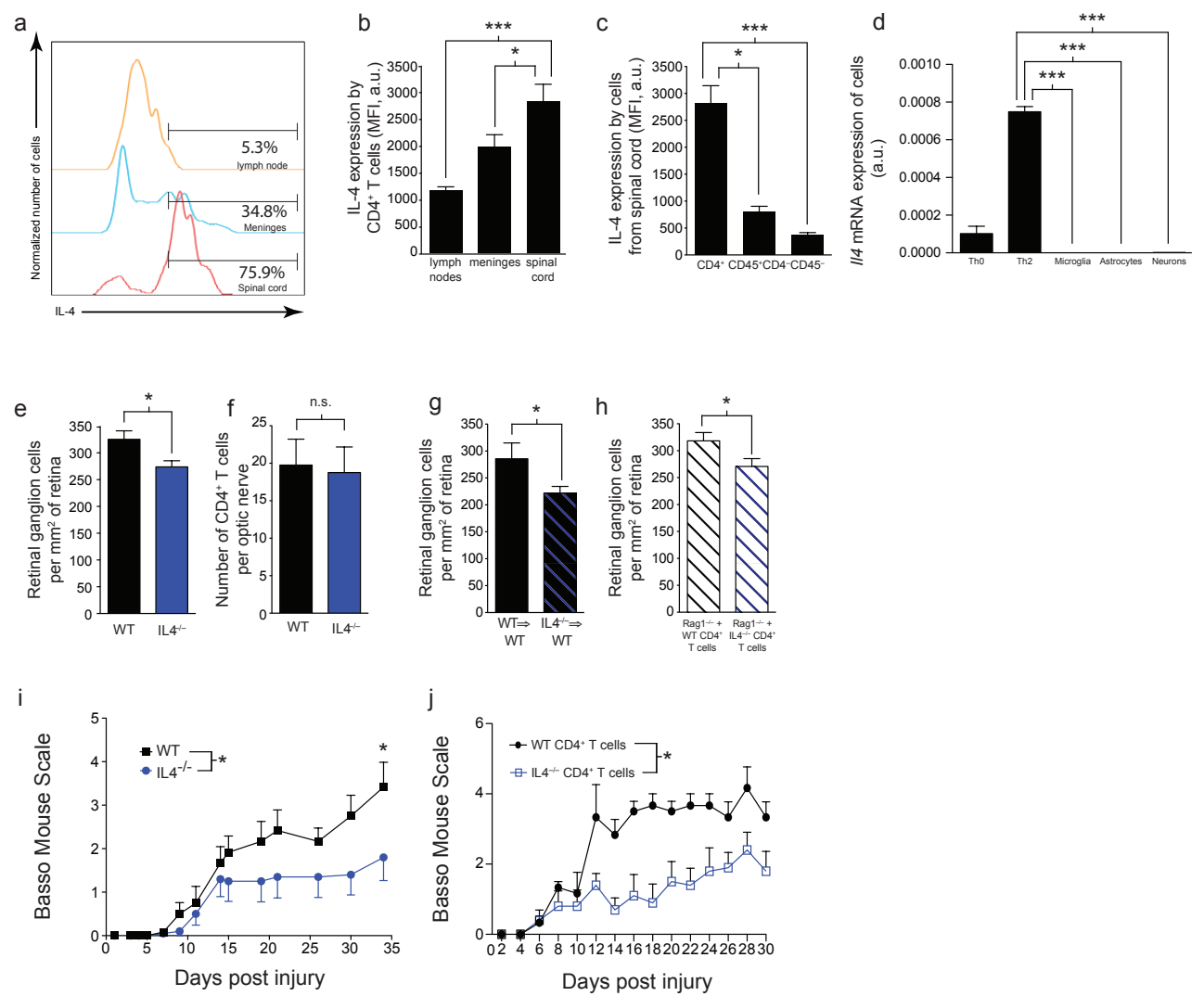

Fig. 2. T cell-derived IL-4 is neuroprotective in CNS trauma. (a, b) High expression of IL-4 in $\mathrm{CD}^{+} \mathrm{T}$ cells in the spinal cord after injury. Representative histograms (a) and quantification (b) of IL-4 expression in $\mathrm{CD}^{+} \mathrm{T}$ cells from the lymph nodes, meninges, and spinal cord of $\mathrm{C} 57 \mathrm{Bl} / 6$ mice after contusion injury $(\mathrm{n}=4$ per group; $* * *, \mathrm{p}<0.001$; *, $\mathrm{p}<0.05$; One-way ANOVA with Bonferroni's post-test; representative of three experiments). (c) $\mathrm{T}$ cells express greater IL-4 than other cells in the spinal cord after injury. Quantification of IL-4 expression in $\mathrm{CD}^{+} \mathrm{T}$ cells, $\mathrm{CD} 45^{+} \mathrm{CD} 4^{-}$leukocytes, or $\mathrm{CD}^{-} 5^{-}$resident $\mathrm{CNS}$ cells in the spinal cord of $\mathrm{C} 57 \mathrm{Bl} / 6$ mice after contusion injury $(\mathrm{n}=$ 
4 per group; ${ }^{* *}, \mathrm{p}<0.001 ; *, \mathrm{p}<0.05$; One-way ANOVA with Bonferroni's post-test; representative of two experiments). (d) $I l 4$ mRNA is expressed by cultured $\mathrm{CD}^{+} \mathrm{T}$ cells, but not by cultured resident cells of the CNS. Il4 mRNA expression of cultured cells, normalized to expression of Gapdh ( $\mathrm{n}=4$ per group; ${ }^{* * *}, \mathrm{p}<0.001$; One-way ANOVA with Bonferroni's post-test; representative of two experiments). (e) $\mathrm{IL}-4^{-/-}$mice have impaired neuronal survival. Bar graphs represent retinal ganglion cell survival (mean \pm s.e.m.) of IL- $4^{-/-}$or wild type mice, assessed 7 days after optic nerve injury by FluoroGold staining $\left(\mathrm{n}=15\right.$, wild type; $\mathrm{n}=10, \mathrm{IL}_{-} 4^{--} ;{ }^{*}, \mathrm{p}<0.05$; Student's t-test; representative of two experiments). (f) $\mathrm{IL}-4^{-/-}$mice have no difference in $\mathrm{T}$ cell infiltration into the injured CNS parenchyma. Bar graphs represent the number of $\mathrm{CD} 4^{+} \mathrm{T}$ cells that infiltrated into the CNS parenchyma of $\mathrm{IL}_{-} 4^{-/-}$or wild type mice $(\mathrm{n}=4$ per group; Student's t-test; representative of two experiments). (g) Mice transplanted with IL$4^{--}$bone marrow have worse neuronal survival than those transplanted with wild type bone marrow. Retinal ganglion cell survival (mean \pm s.e.m.) of wild type $\Rightarrow$ wild type or IL- $4^{--} \Rightarrow$ wild type bone marrow chimeras. Bone marrow was allowed to engraft for 6 weeks before optic nerve injury, and retinal ganglion cell survival was assessed 7 days after injury by Fluoro-Gold staining $\left(n=7\right.$, wild type bone marrow; $n=6, \mathrm{IL}_{-} 4^{-/-}$bone marrow; *, $\mathrm{p}<0.05$; Student's t-test; representative of two experiments). (h) $\operatorname{Rag} 1^{-/-}$ mice receiving $\mathrm{IL}_{-} 4^{-/-} \mathrm{CD} 4^{+} \mathrm{T}$ cells have impaired neuronal survival from $\mathrm{Rag} 1^{-/}$mice receiving wild type $\mathrm{T}$ cells. Retinal ganglion cell survival (mean \pm s.e.m.) of Rag ${ }^{-/}$mice injected with either wild type or IL-4 ${ }^{-/} \mathrm{CD} 4^{+} \mathrm{T}$ cells 3 weeks before optic nerve injury. Retinal ganglion cell survival was quantified 7 days after injury by Fluoro-Gold staining $\left(\mathrm{n}=13\right.$ wild type and IL- $4^{-/-} \mathrm{T}$ cell injected; *, $\mathrm{p}<0.05$; Student's t-test; representative 
of two experiments). (i) $\mathrm{IL}-4^{-/-}$mice have impaired functional recover after spinal cord injury. Locomotor score of wild type and IL- $4^{-/-}$mice that have undergone a spinal cord injury, as assessed by a blinded observer using the Basso Mouse Scale ( $\mathrm{n}=6$, wild type; $\mathrm{n}=10, \mathrm{IL}-4^{--} ; *, \mathrm{p}<0.05$; Repeated-measure two-way ANOVA with Bonferroni's posttest; representative of two experiments). (j) Rag $1^{-/-}$mice reconstituted with $\mathrm{IL}-4^{-/-} \mathrm{T}$ cells have less functional recovery than $\operatorname{Rag} 1^{-/-}$mice reconstituted with wild type $\mathrm{CD} 4^{+} \mathrm{T}$ cells. Locomotor score of Rag $1^{-/}$mice reconstituted with either wild type or IL-4 ${ }^{-/}$ $\mathrm{CD}^{+} \mathrm{T}$ cells from naïve mice that have undergone a spinal cord injury, as assessed by a blinded observer using the Basso Mouse Scale ( $\mathrm{n}=4$ per group; *, $\mathrm{p}<0.05$; Repeatedmeasure two-way ANOVA with Bonferroni's post-test; representative of two experiments). 

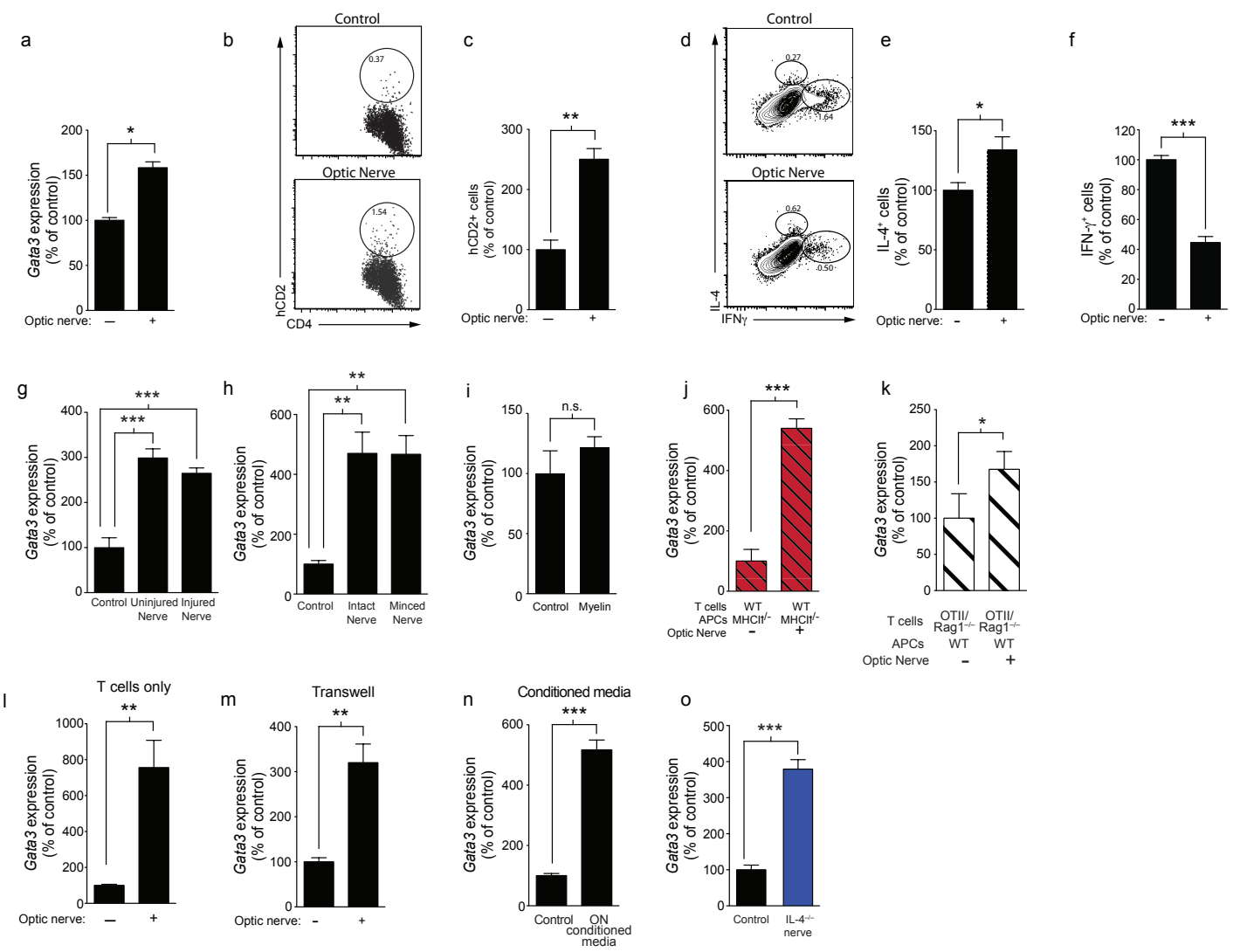

Fig. 3. Molecular signals from CNS tissue skew T cells toward a Th2 phenotype. (a) Optic nerves upregulate Gata3 in $\mathrm{CD}^{+} \mathrm{T}$ cells. Negatively sorted $\mathrm{CD} 4^{+} \mathrm{T}$ cells from total lymph nodes were incubated with optic nerves and APCs. Three optic nerves were used in each well and the co-cultures were incubated for 72 hours, after which $\mathrm{CD} 4^{+} \mathrm{T}$ cells were re-sorted and Gata3 expression analyzed using qRT-PCR. Bar graphs represent Gata3 expression (relative to Gapdh; mean \pm s.e.m.) in $\mathrm{CD}^{+}$T cells (n $=3$ per group; *, $\mathrm{p}<0.05$; Student's t-test; representative of $>$ three experiments). (b, c) Optic nerves induce hCD2 production in $\mathrm{CD}^{+} \mathrm{T}$ cells in $\mathrm{KN} 2$ reporter mice. Representative flow cytometry plots (b) and quantification (mean \pm s.e.m., c) of hCD2 expression (reporter for IL-4 protein) in cultures of lymph node cells incubated with or without optic 
nerves for 4 days $(\mathrm{n}=5$, control; $\mathrm{n}=3$, optic nerve; **, $\mathrm{p}<0.01$; Student's t-test; representative of two experiments). (d, e) Optic nerves induce IL-4 production in $\mathrm{CD} 4^{+} \mathrm{T}$ cells. Representative flow cytometry plots $(\mathbf{d})$ and quantification (mean \pm s.e.m., e) of IL$4^{+} \mathrm{CD} 4^{+} \mathrm{T}$ cells in cultures of lymph node cells incubated with or without optic nerves for 5 days, as a percentage of $\mathrm{CD}^{+} \mathrm{T}$ cells $\left(\mathrm{n}=13\right.$, control; $\mathrm{n}=13$, optic nerve; ${ }^{*}, \mathrm{p}<$ 0.05; Student's t-test; representative of two experiments). (f) Optic nerves decrease IFN $\gamma$ production in $\mathrm{CD}^{+} \mathrm{T}$ cells. Quantification (mean \pm s.e.m.) of $\mathrm{IFN}^{+} \mathrm{CD}^{+} \mathrm{T}$ cells in cultures of lymph node cells incubated with or without optic nerves for 5 days, as a percentage of $\mathrm{CD}^{+} \mathrm{T}$ cells $(\mathrm{n}=13$, control; $\mathrm{n}=13$, optic nerve; ***, $\mathrm{p}<0.001$; Student's t-test; representative of $>$ three experiments). (g) Optic nerves from previously injured or naïve animals are able to upregulate Gata3 in $\mathrm{CD}^{+} \mathrm{T}$ cells. Gata3 expression (mean \pm s.e.m.) in sorted $\mathrm{CD}^{+} \mathrm{T}$ cells after 72 hours of culture in the presence or absence of optic nerves isolated from either injured (three days after injury) or naïve mice ( $\mathrm{n}=4$ per group; $* * *, \mathrm{p}<0.001$; One-way ANOVA with Bonferroni's post-test; representative of two experiments). (h) Additional physical damage to the optic nerves does not increase Gata3 beyond damage from excision. Gata3 expression (mean \pm s.e.m.) in sorted $\mathrm{CD} 4^{+} \mathrm{T}$ cells after 72 hours of culture in the presence or absence of optic nerves that had been minced or left intact $(\mathrm{n}=3$ per group; **, $\mathrm{p}<0.01$; One-way ANOVA with Bonferroni's post-test; representative of two experiments). (i) Myelin vesicles do not upregulate Gata3 in $\mathrm{CD}^{+} \mathrm{T}$ cells. $\mathrm{CD}^{+} \mathrm{T}$ cells from deep cervical lymph nodes and APCs were incubated in the presence or absence of $80 \mu \mathrm{g}$ of myelin vesicles for 72 hours before $\mathrm{CD}^{+} \mathrm{T}$ cells were re-sorted and assayed for Gata3 relative to Gapdh $(\mathrm{n}=3$ per condition; $\mathrm{p}>0.5$; Student's t-test; representative of two experiments). (j) Optic nerves 
can upregulate Gata3 when wild type $\mathrm{CD}^{+} \mathrm{T}$ cells are incubated with $\mathrm{MHCII}^{-/-}$antigen presenting cells. Gata 3 expression (mean \pm s.e.m.) in sorted wild type $\mathrm{CD} 4^{+} \mathrm{T}$ cells cocultured with $\mathrm{MHCII}^{-/}$antigen presenting cells in the presence or absence of optic nerves from uninjured mice $\left(\mathrm{n}=3\right.$ per group; ${ }^{* * *}, \mathrm{p}<0.001$, Student's $\mathrm{t}$-test; representative of two experiments). (k) Optic nerves can upregulate Gata3 when OTII/Rag1 ${ }^{-/} \mathrm{CD}^{+} \mathrm{T}$ cells are incubated with wild-type antigen presenting cells. Gata3 expression (mean \pm s.e.m.) in sorted OTII/Rag1 ${ }^{-/} \mathrm{CD} 4^{+} \mathrm{T}$ cells co-cultured with wild-type antigen presenting cells in the presence or absence of optic nerves from uninjured mice $(n=3$ per group; *, $\mathrm{p}<0.05$, Student's t-test; representative of two experiments) (l) Optic nerves can upregulate Gata3 when incubated with wild type $\mathrm{CD} 4^{+} \mathrm{T}$ cells alone. Gata3 expression (mean \pm s.e.m.) in sorted wild type $\mathrm{CD}^{+} \mathrm{T}$ cell cultures lacking antigen presenting cells incubated for 72 hours in the presence or absence of optic nerves from uninjured mice (n $=3$ per group; ${ }^{* *}, \mathrm{p}<0.001$; Student's t-test; representative of $>$ three experiments). (m) Optic nerves upregulate Gata3 in $\mathrm{CD}^{+} \mathrm{T}$ cells across a transwell. Gata3 expression (mean \pm s.e.m.) in sorted $\mathrm{CD}^{+} \mathrm{T}$ cells after 72 hours of culture in the presence or absence of isolated optic nerves placed in a transwell with $0.4 \mu \mathrm{m}$ pores $(\mathrm{n}=3$ per group; **, $\mathrm{p}<0.01$; Student's t-test; representative of two experiments). (n) Optic nerves conditioned media is sufficient to upregulate Gata 3 in $\mathrm{CD}^{+} \mathrm{T}$ cells. Gata3 expression (mean \pm s.e.m.) in sorted $\mathrm{CD}^{+} \mathrm{T}$ cells after 72 hours of culture in optic nerve conditioned media or control media $\left(\mathrm{n}=5\right.$ per group; ${ }^{* *}, \mathrm{p}<0.001$; Student's t-test; representative of two experiments). (o) $\mathrm{IL}_{-} 4^{-/-}$optic nerves are able to upregulate Gata3 expression in $\mathrm{CD}^{+} \mathrm{T}$ cells. Gata3 expression (mean \pm s.e.m.) in sorted $\mathrm{CD} 4^{+} \mathrm{T}$ cells after 72 hours of culture in the presence or absence of isolated optic nerves from IL- $4^{-/-}$ 
114

mice $(\mathrm{n}=4$ per group; $* * *, \mathrm{p}<0.001$; Student's $\mathrm{t}$-test; representative of two experiments). 

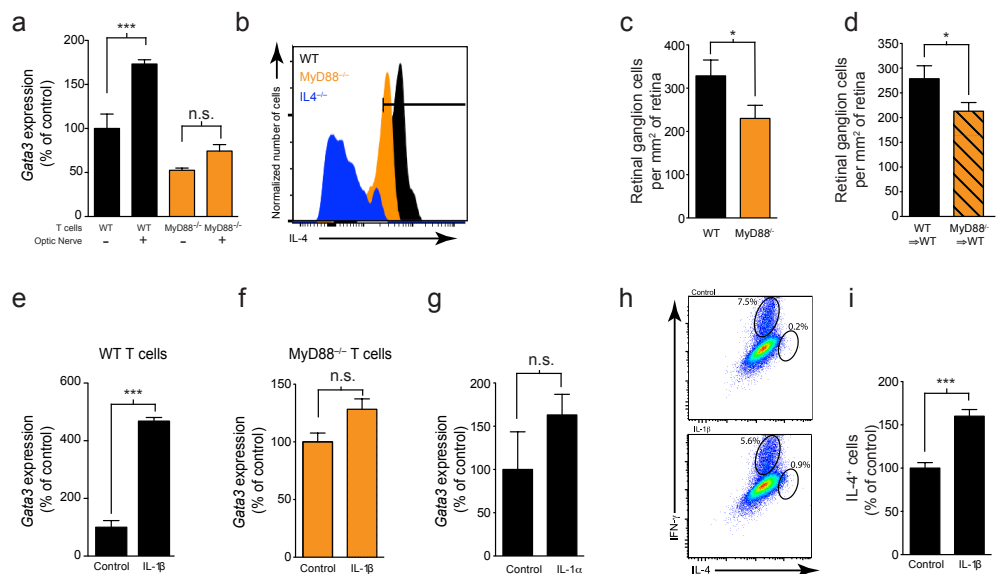

f

g

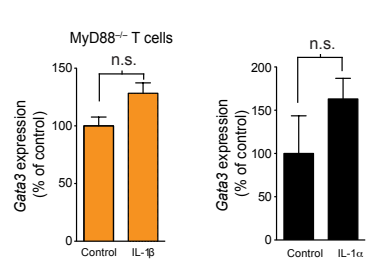

h
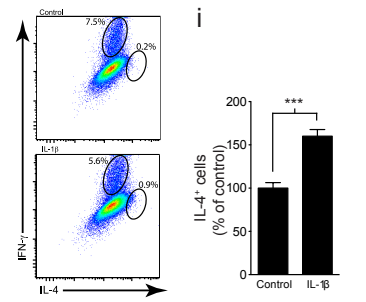

j

$\mathrm{k}$

I

$\mathrm{m}$
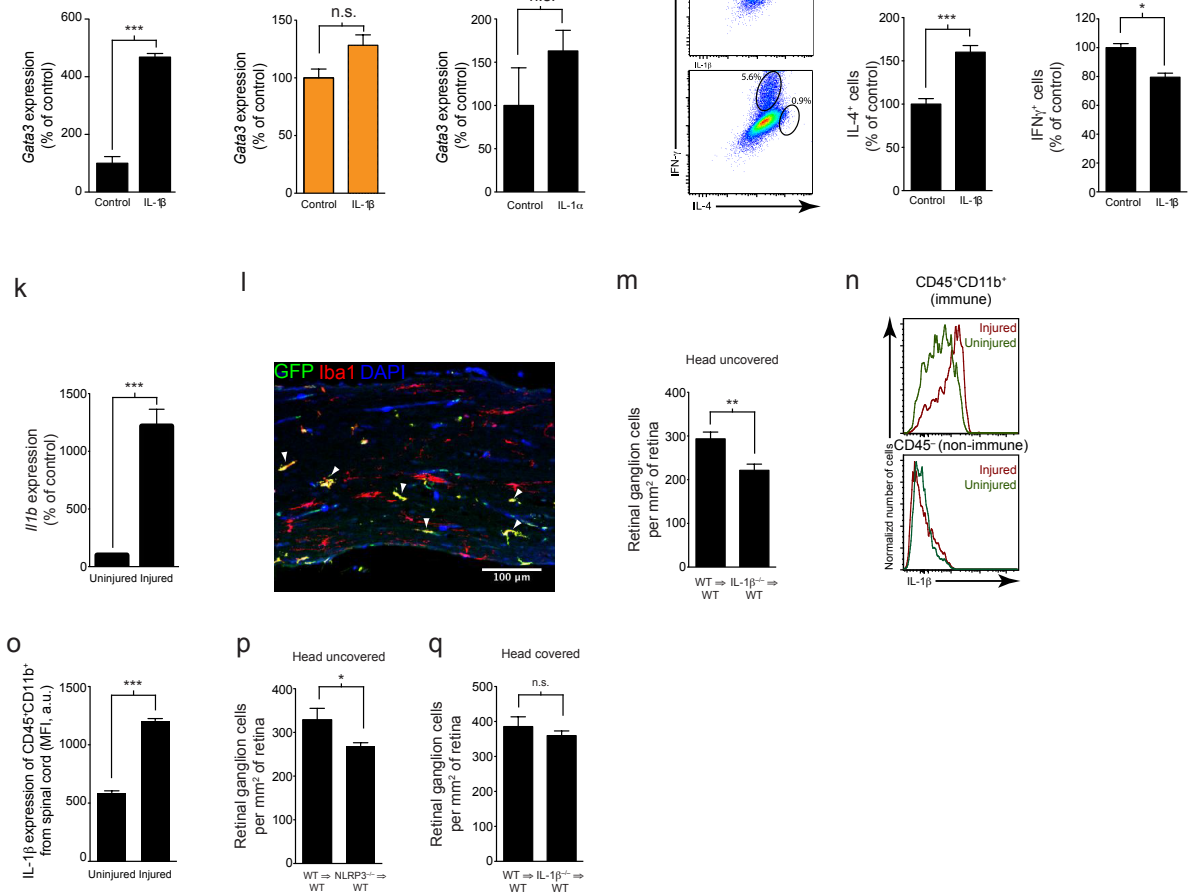

Fig. 4. IL-1 $\beta$ leads to Th2 skewing of $T$ cells and is important for neuronal survival after CNS injury (a) Optic nerves are unable to upregulate Gata3 in MyD88 ${ }^{-/} \mathrm{T}$ cells

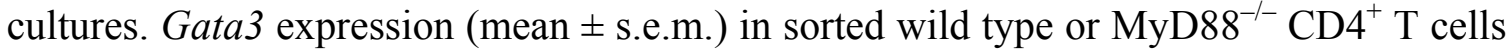


cultured in the presence or absence of optic nerves from uninjured mice $(n=3$ per group; ***, $\mathrm{p}<0.001$; Student's t-test; representative of two experiments). (b) IL-4 expression

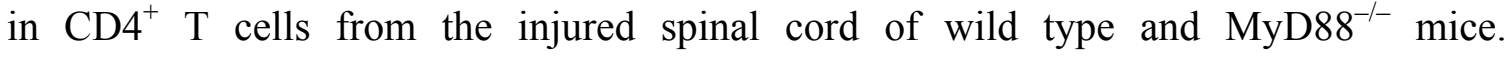
Representative histograms of IL-4 expression in $\mathrm{CD}^{+} \mathrm{T}$ cells from the spinal cord of $\mathrm{C} 57 \mathrm{~B} 1 / 6, \mathrm{MyD}^{-/-} 8^{-}$, or $\mathrm{IL}_{-} 4^{-/-}$mice after contusion injury (representative of two experiments). (c) MyD88 ${ }^{-/}$mice have decreased neuronal survival after optic nerve crush injury. Retinal ganglion cell survival (mean \pm s.e.m.) of wild type and MyD88 $8^{-/-}$ mice 7 days after optic nerve injury as assessed by Fluoro-Gold staining $(\mathrm{n}=11$, wild type; $\mathrm{n}=11$, MyD88 $^{--} ; *, \mathrm{p}<0.05$; Student's t-test; representative of two experiments). (d) Mice receiving MyD88 $8^{-/-}$bone marrow display impaired neuronal survival from those receiving wild type bone marrow. Retinal ganglion cell survival (mean \pm s.e.m.) of wild type $\Rightarrow$ wild type or MyD88 $8^{-/-} \Rightarrow$ wild type bone marrow chimeras. Bone marrow was allowed to engraft for 6 weeks before optic nerve injury, and retinal ganglion cell survival was assessed 7 days after injury by Fluoro-Gold staining $(\mathrm{n}=6$, wild type bone marrow recipients; $\mathrm{n}=7 \mathrm{MyD} 88^{-/-}$bone marrow recipients; * $\mathrm{p}<0.05$; Student's t-test; representative of two experiments). (e) IL-1 $\beta$ increases Gata 3 mRNA expression in wildtype $\mathrm{CD}^{+} \mathrm{T}$ cells. Gata3 expression (mean \pm s.e.m.) in $\mathrm{CD} 4^{+} \mathrm{T}$ cells after 72 hours of culture in the presence or absence of $10 \mathrm{ng} / \mathrm{mL}$ IL-1 $\beta$ ( $\mathrm{n}=3$ per group; ${ }^{* * *}, \mathrm{p}<0.001$; Student's t-test; representative of three experiments). (f) IL-1 $\beta$ does not increase Gata3

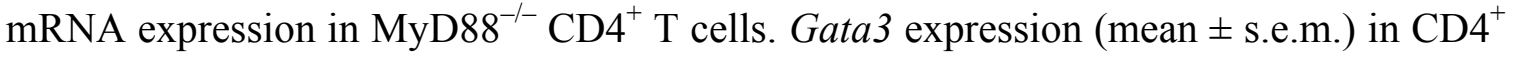
T cells after 72 hours of culture in the presence or absence of $10 \mathrm{ng} / \mathrm{mL}$ IL-1 $\beta$ ( $\mathrm{n}=3$ per group; Student's t-test; representative of two experiments). (g) IL-1 $\alpha$ has a marginal effect on Gata 3 mRNA expression in $\mathrm{CD}^{+} \mathrm{T}$ cells. Gata 3 expression (mean \pm s.e.m.) in 
$\mathrm{CD}^{+} \mathrm{T}$ cells after 72 hours of culture in the presence or absence of $10 \mathrm{ng} / \mathrm{mL}$ IL- $1 \alpha(\mathrm{n}=$ 3 per group; Student's t-test; representative of two experiments). (h, i) IL-1 $\beta$ induces IL4 production in $\mathrm{CD}^{+} \mathrm{T}$ cells. Representative flow cytometry plots $(\mathbf{h})$ and quantification (mean \pm s.e.m., i) of IL-4 ${ }^{+} \mathrm{CD} 4^{+} \mathrm{T}$ cells in cultures of lymph node cells incubated with or without $10 \mathrm{ng} / \mathrm{mL}$ IL- $1 \beta$ for 5 days, as a percentage of $\mathrm{CD}^{+} \mathrm{T}$ cells $(\mathrm{n}=6$, control; $\mathrm{n}=$ 6, IL-1 $\beta ; * * *, p<0.001 ;$ Student's t-test; representative of two experiments). (j) IL-1 $\beta$ decreases IFN $\gamma$ production in $\mathrm{CD}^{+} \mathrm{T}$ cells. Quantification (mean \pm s.e.m.) of $\mathrm{IFN}^{+}$ $\mathrm{CD}^{+} \mathrm{T}$ cells in cultures of lymph node cells incubated with or without IL-1 $\beta$ for 5 days, as a percentage of $\mathrm{CD} 4^{+} \mathrm{T}$ cells $\left(\mathrm{n}=6\right.$, control; $\mathrm{n}=6$, optic nerve; ${ }^{*}, \mathrm{p}<0.05$; Student's t-test; representative of three experiments). (k) $I l l b$ mRNA increases in the injured CNS. $I l 1 b$ mRNA expression in optic nerves that were uninjured or 1 day after injury $(\mathrm{n}=4$, uninjured; $\mathrm{n}=5$, injured; ${ }^{* *}, \mathrm{p}<0.001$; Student's t-test; representative of two experiments). (I) After head-exposed irradiation, bone-marrow derived cells engraft in the optic nerve. Representative images of uninjured optic nerves of $U b c$-GFP $\Rightarrow$ wild type bone marrow chimeras stained for Iba1, showing infiltration of $\mathrm{GFP}^{+}$myeloid cells into the uninjured optic nerve 6 weeks after bone marrow irradiation. Arrowheads indicate $\mathrm{GFP}^{+} \mathrm{Iba}^{+}$myeloid cells that have infiltrated into the tissue, and arrows indicate $\mathrm{GFP}^{-}$ Iba $1^{+}$microglia that were not replaced after irradiation. (m) Chimeric mice that receive IL- $1 \beta^{-/-}$bone marrow have decreased neuronal survival compared to chimeric mice that receive wild-type bone marrow. Retinal ganglion cell survival (mean \pm s.e.m.) of wild type $\Rightarrow$ wild type or IL- $1 \beta^{-/-} \Rightarrow$ wild type bone marrow chimeras after head-exposed lethal irradiation. Bone marrow was allowed to engraft for 6 weeks before optic nerve injury, and retinal ganglion cell survival was assessed 7 days after injury by Fluoro-Gold 
staining $\left(\mathrm{n}=12\right.$, wild type bone marrow recipients; $\mathrm{n}=12$, IL- $1 \beta^{-/-}$bone marrow recipients; ${ }^{* *}, \mathrm{p}<0.01 ;$ Student's t-test; representative of two experiments). (n, o) IL-1 $\beta$ is increased in myeloid cells after injury, but not in CNS-resident cells. Representative flow cytometry histograms (n) and quantification (o) of IL-1 $\beta$ protein expression one day after injury in $\mathrm{CD} 45^{+} \mathrm{CD} 11 \mathrm{~b}^{+}$or $\mathrm{CD} 45^{-}$cells in the spinal cord $(\mathrm{n}=4$, uninjured; $\mathrm{n}=4$ injured; ***, p $<0.001$; Student's t-test; representative of two experiments). (p) Chimeric mice that receive $\mathrm{NLRP}^{-/-}$bone marrow have decreased neuronal survival compared to chimeric mice that receive wild-type bone marrow. Retinal ganglion cell survival (mean \pm s.e.m.) of wild type $\Rightarrow$ wild type or $\mathrm{NLRP}^{-/-} \Rightarrow$ wild type bone marrow chimeras after head-exposed lethal irradiation. Bone marrow was allowed to engraft for 6 weeks before optic nerve injury, and retinal ganglion cell survival was assessed 7 days after injury by Fluoro-Gold staining $\left(\mathrm{n}=10\right.$, wild type bone marrow recipients; $\mathrm{n}=9, \mathrm{NLRP}^{-/-}$bone marrow recipients; *, $\mathrm{p}<0.05$; Student's t-test; representative of two experiments). (q) Chimeric mice that receive IL-1 $\beta^{-/-}$bone marrow after head-covered irradiation have no difference in neuronal survival compared to chimeric mice that receive wild-type bone marrow. Retinal ganglion cell survival (mean \pm s.e.m.) of wild type $\Rightarrow$ wild type or IL$1 \beta^{-/-} \Rightarrow$ wild type bone marrow chimeras after head-covered lethal irradiation. Bone marrow was allowed to engraft for 6 weeks before optic nerve injury, and retinal ganglion cell survival was assessed 7 days after injury by Fluoro-Gold staining $(\mathrm{n}=7$, wild type bone marrow recipients; $\mathrm{n}=5, \mathrm{IL}-1 \beta^{-/-}$bone marrow recipients; Student's ttest; representative of two experiments). 

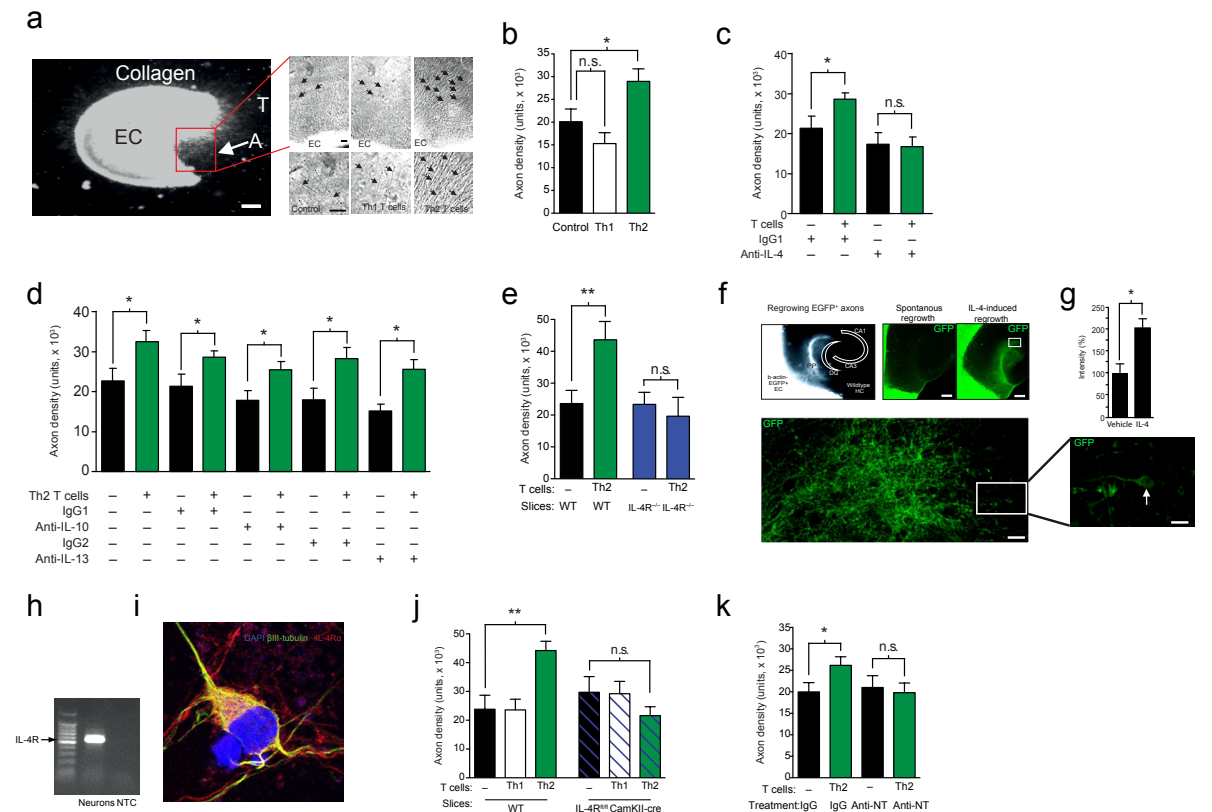

$\mathrm{k}$
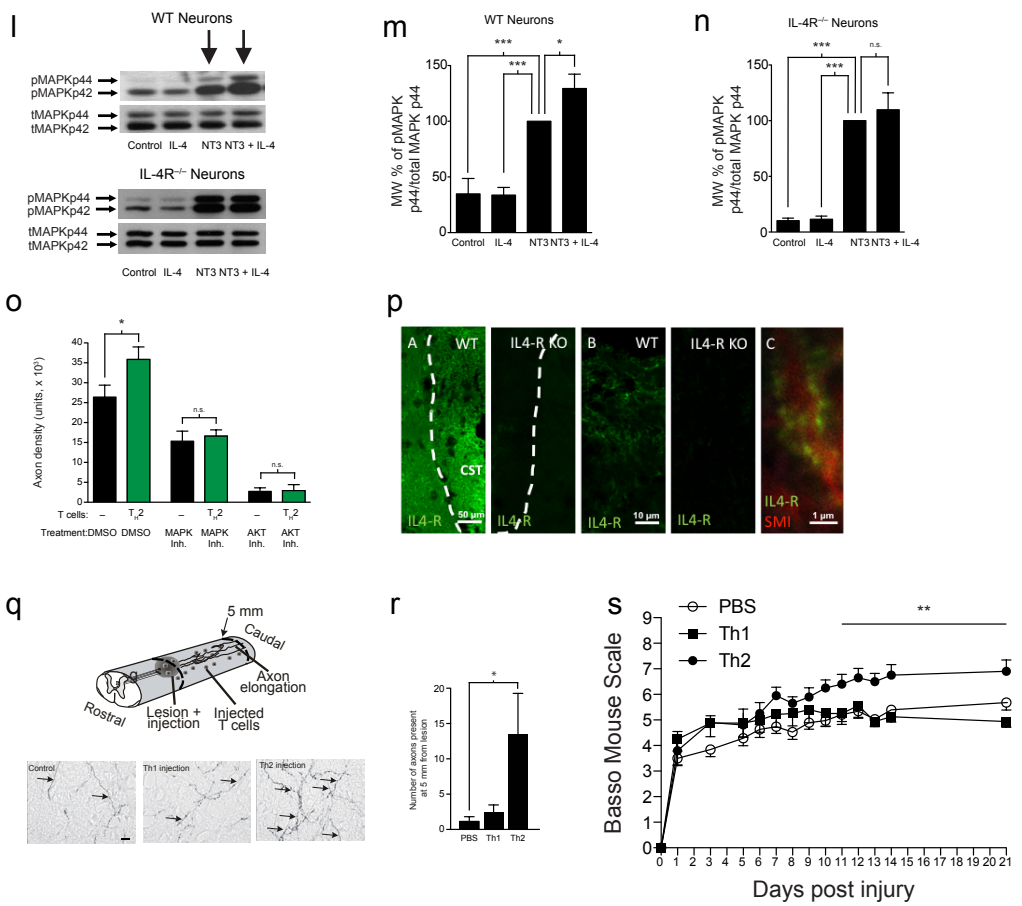

Figure 5: $T$ cell derived IL-4 acts on neurons to induce axonal regrowth. (a) Outgrowth assay (see Materials \& Methods for details); the cortical explant culture (EC) was embedded in a three-dimensional collagen I gel matrix; the concave side of the EC 
with outgrowing axons (A) is oriented towards the T cells (T). Scale bar: $300 \mu \mathrm{m}$. (b) ConA-activated Th2 cells significantly increased axon density, while ConA-activated Th1 cells did not (mean \pm s.e.m.; $\mathrm{n}=29$, control; $\mathrm{n}=33$, Th1; $\mathrm{n}=29$, Th2; * $\mathrm{p}<0.05$; One-way ANOVA with Bonferroni's post-test). (c) Co-culture of wild type slices with activated Th2 cells stimulates axonal outgrowth, which is inhibited by the addition of neutralizing antibodies to IL-4 $(\mathrm{n}=24, \operatorname{IgG} 1 ; \mathrm{n}=22, \operatorname{IgG} 1+\mathrm{Th} 2 ; \mathrm{n}=22$, anti-IL-4; $\mathrm{n}=$ 25, anti-IL-4 + Th2; *, p $<0.05$; One-way ANOVA with Bonferroni's post-test). (d) Inhibition of IL-10 or IL-13 does not affect neuronal outgrowth from slices induced by Th2 cells. Slices were incubated with Th2 cells in the presence or absence of blocking antibodies to IL-10, IL-13, or their corresponding isotype controls. Bar graph represents axonal density regrown from slices $\left(\mathrm{n}>12\right.$ per group; ${ }^{*}, \mathrm{p}<0.05$; One-way ANOVA with Bonferroni's post-test). (e) Co-culture of wild type brain slices with Th2 cells stimulated axon density (mean \pm s.e.m.; $\mathrm{n}=18$, control; $\mathrm{n}=17$, Th2; $* \mathrm{p}<0.05$, Student's t-test), however, axon growth of slices derived from IL-4R knockout (IL-4R ${ }^{--}$) mice was unchanged in the presence or absence of Th2 cells $\left(n=12, I_{4} 4 R^{--} ; n=12\right.$, IL$4 \mathrm{R}^{-/-}+\mathrm{Th} 2 ; * *, \mathrm{p}<0.01$; One-way ANOVA with Bonferroni's post-test). (f) Axon ingrowth co-culture model of the entorhinal cortex and hippocampus in vitro (for details, see (Hechler et al., 2010). Entorhinal cortex (EC) explants of mice, which express EGFP under control of the $\beta$-actin promoter, were co-cultivated with a wildtype hippocampus (HC). The perforant path (PP) fibers originating from $\mathrm{EGFP}^{+}$- EC-slices reinnervated the dentate gyrus (DG), and regenerating axons display growth cones in the reinnervated tissue. CA: cornu ammonis. White boxes indicate areas shown in higher magnification.

Scale bars: $300 \mu \mathrm{m}, 15 \mu \mathrm{m}$, and $3 \mu \mathrm{m}$. (g) Quantification (mean \pm s.e.m.) of the EGFP ${ }^{+}$ 
area in the hippocampus indicated a statistically significant stimulation of axon ingrowth in the presence of recombinant IL-4 $(500 \mathrm{ng} / \mathrm{ml}) .(\mathrm{n}=28$, vehicle; $\mathrm{n}=28$; IL-4 treated; **, $\mathrm{p}<0.01$; Student's t-test) (h) RT-PCR of primary neurons showing expression of IL4R $\alpha$ message $(\mathrm{NTC}=$ no template control). (i) Immunofluorescent staining of cultured neurons, showing IL-4R $\alpha$ expression on $\beta$-III tubulin positive neurons. (j) Th2 cells are not able to elicit increase in axon density from slices obtained from IL- $4 \mathrm{R}^{\text {flflf }}:$ CamKII ${ }^{\text {cre }}$ mice (lacking the IL-4-receptor exclusively on neurons), unlike their effect on wild type slices $\left(\mathrm{n}=30\right.$, control; $\mathrm{n}=30, \mathrm{Th} 1 ; \mathrm{n}=28, \mathrm{Th} 2 ; \mathrm{n}=30, \mathrm{IL}-4 \mathrm{R}^{\text {flflf }}: \mathrm{CamKII}^{\mathrm{cre}} ; \mathrm{n}=32$, IL$4 \mathrm{R}^{f l f l}:$ CamKII $^{\text {cre }}+\mathrm{Th} 1 ; \mathrm{n}=30, \mathrm{IL}^{\prime}-4 \mathrm{R}^{f l f l}: \mathrm{CamKII}^{\text {cre }}+\mathrm{Th} 2 ; * *, \mathrm{p}<0.01$; One-way ANOVA with Bonferroni's post-test). (k) Combined inhibition of NT-3, NT-4 and NGF by specific antibodies abolished the Th2 cell-induced increase of axonal density $(n=27$, control; $\mathrm{n}=30$, Th2; $\mathrm{n}=25$, anti-NT; $\mathrm{n}=26$, anti-NT $+\mathrm{Th} 2 ;{ }^{*}, \mathrm{p}<0.05$; Student's ttest). (I-n) IL-4 potentiates pMAPK signaling in wild-type cultured neurons, but not in IL-4R ${ }^{-/-}$neuronal cultures. Representative blots (I) and quantification of pMAPK44 in wild type (m) and IL-4R $\mathrm{R}^{-/-}$neuronal cultures (n) that have been treated with IL-4 and/or NT3 before analysis by western blot $(*: \mathrm{p}<0.05 ; * * *: \mathrm{p}<0.001$ ( $\mathrm{n}=11$ wild type, $\mathrm{n}=9$ IL-4R ${ }^{-/}$), One-way ANOVA with Bonferroni's post-test. (o) Inhibition of the MAPK or AKT by small molecule inhibitors abolished the Th2 cell-induced increase of axonal density ( $\mathrm{n}=8-33 ; *, \mathrm{p}<0.05$; Student's t-test). (p) Cortico-spinal tract (CST) axons express IL4-R $\alpha$. Horizontal sections of the spinal cord revealed specific IL4-R expression in a region which mainly contains CST-axons in wild-type animals (left) while no staining was observed in IL4-R KO animals (right). B. Higher magnification of the IL4-R $\alpha$ positive structures observed in wild-type animals displayed tubular structures 
with a clear delineation of the presumed axonal membrane (left) while no signal was detected in IL4-R KO animals (right) C. Co-staining for SMI 312, a pan-axonal neurofilament marker, confirmed the axonal origin of the tubular structures. Scale bars: 50um (A), 10um (B), 1um (C). (q) Schematic drawing of the injection of Th2 T cells, which promote axonal regrowth after spinal cord injury, and representative photomicrographs show regrowing nerve fibers labeled with biotinylated dextran amine. (r) The quantitative analysis of axonal sprouting/regrowth in spinal cord-injured mice injected with Th1 or Th2 T cells, showing a significant increase of labeled nerve fibers after spinal cord injury in Th2 injected mice $(n=10$, PBS; $n=8$, Th1; $n=9$, Th2; *, $p<$ 0.05; One-way ANOVA with Bonferroni's post-test). (s) T cells promote axonal regeneration after spinal cord injury in vivo. Locomotion analysis using the Basso Mouse Scale showed a significant long term improvement of neurological outcome after SCI by intralesional injections of IL- $4^{+}$cells during the indicated period $(n=20, P B S ; n=9$, Th2 T cells; $\mathrm{n}=8$ Th1 T cells; **, $\mathrm{p}<0.01,2$-way ANOVA). 


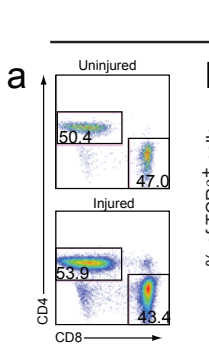

dCLNs (CD4 vs. CD8)
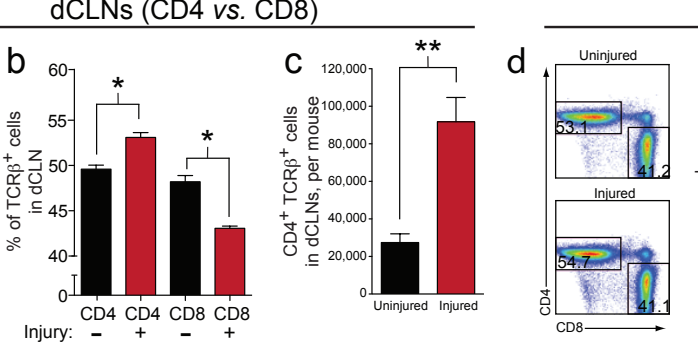

SDLNs (CD4 vs. CD8)
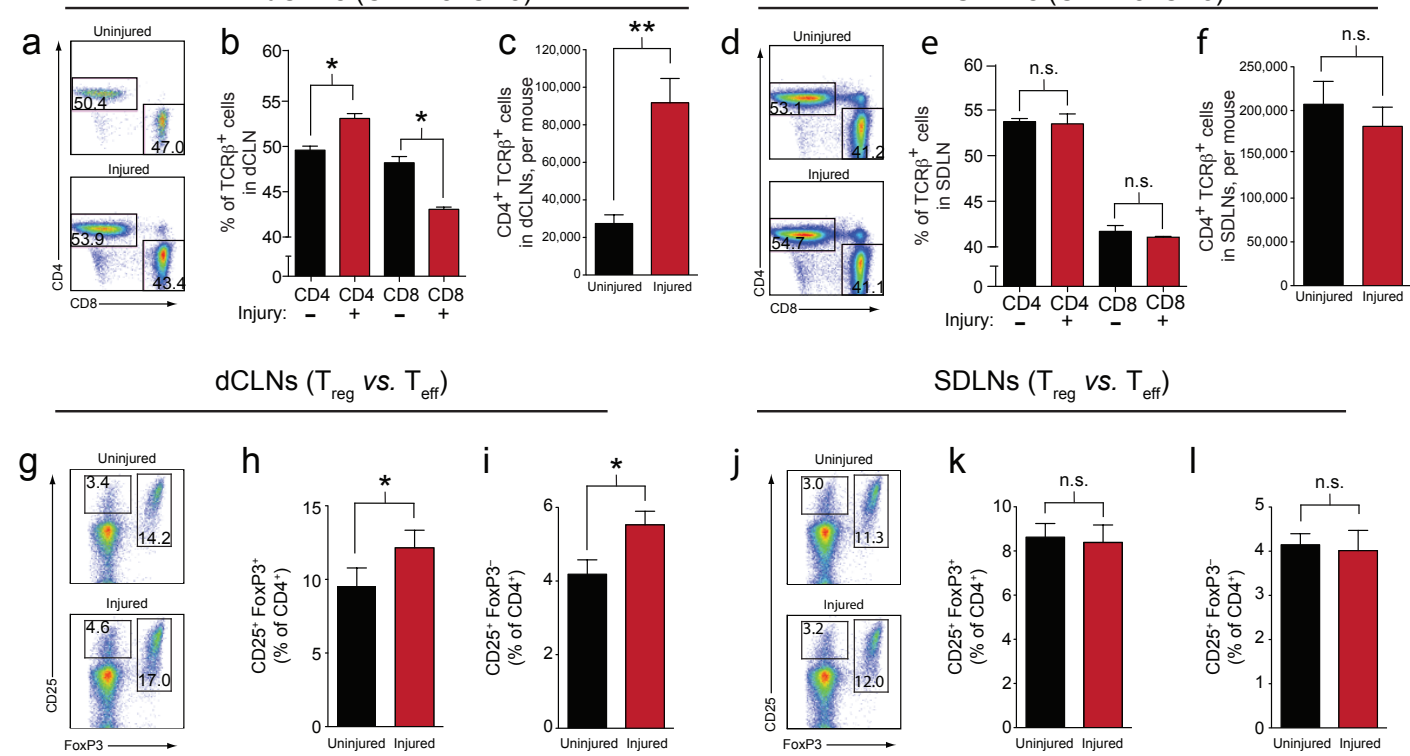

dCLNs $\left(T_{\text {reg }} v s . T_{\text {eff }}\right)$
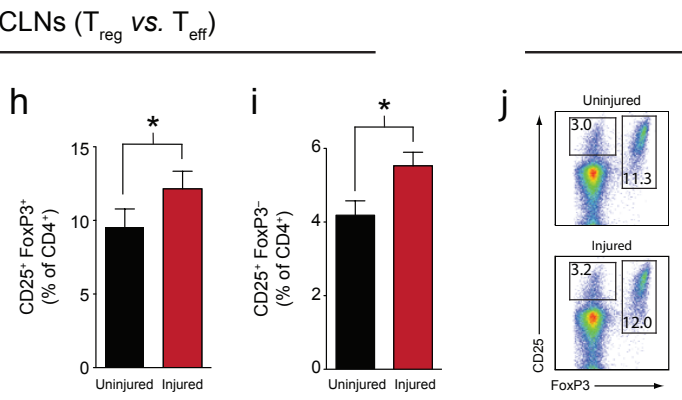

$\operatorname{SDLNs}\left(\mathrm{T}_{\text {reg }} v s . \mathrm{T}_{\text {eff }}\right)$
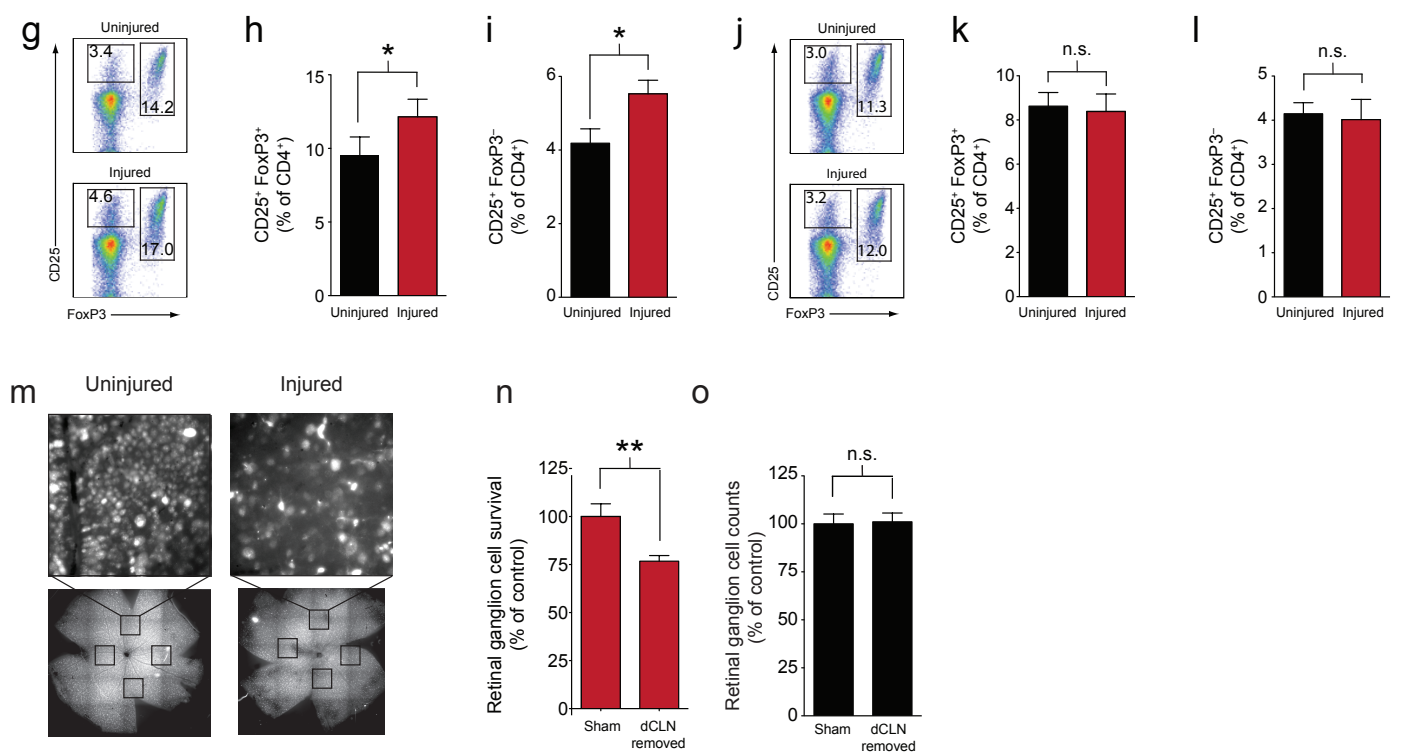

Figure 6: The deep cervical lymph nodes display an immune response after CNS injury and their resection exacerbates neuronal survival. (a) Flow cytometry of CD4 ${ }^{+}$and $\mathrm{CD} 8^{+}$ lymphocytes in the deep cervical lymph nodes 5 days post-injury. Numbers indicate percent $\mathrm{CD}^{+}$(upper left) and $\mathrm{CD}^{+}$(lower right), as a percentage of $\mathrm{TCRb}^{+}$cells. (b, c) Frequency of $\mathrm{CD}^{+}$and $\mathrm{CD} 8^{+}$as a percent of $\mathrm{TCR} \beta^{+}$lymphocytes (b) and number of $\mathrm{CD}^{+} \mathrm{TCRb}^{+} \mathrm{T}$ cells (c) in the deep cervical lymph nodes, as quantified by flow cytometry 5 days after injury $\left(\mathrm{n}=3\right.$ per group; ${ }^{*}, \mathrm{p}<0.05 ;{ }^{*}, \mathrm{p}<0.01$; Student's t-test; representative of $>3$ experiments). (d) Flow cytometry of $\mathrm{CD}^{+}$and $\mathrm{CD} 8^{+}$lymphocytes in the skin-draining lymph nodes 5 days post-injury. Numbers indicate percent CD4 ${ }^{+}$ 
(upper left) and $\mathrm{CD}^{+}$(lower right), as a percentage of $\mathrm{TCRb}^{+}$cells. (e, f) Frequency of $\mathrm{CD}^{+}$and $\mathrm{CD} 8^{+} \mathrm{T}$ cells, as a percent of $\mathrm{TCRb}^{+}$cells $(\mathbf{e})$ and number of $\mathrm{CD}^{+} \mathrm{T}$ cells (f) in the skin draining lymph nodes 5 days post-injury, as quantified by flow cytometry $(\mathrm{n}=$ 3 per group; Student's t-test; representative of $>3$ experiments) (g) Flow cytometry of $\mathrm{CD} 4{ }^{+}$lymphocytes in the deep cervical lymph node 5 days post-injury. Numbers indicate percent activated $\mathrm{T}_{\text {eff }}$ (upper left) and $\mathrm{T}_{\text {reg }}$ (right) cells, as a percentage of $\mathrm{CD} 4^{+}$cells. (h, i) Frequency of $T_{\text {reg }}(\mathbf{h})$ and $T_{\text {eff }}(\mathbf{i})$ cells in the dCLNs as a percent of the uninjured dCLN $(\mathrm{n}=6$ per group; $*, \mathrm{p}<0.05$, Student's t-test, representative of two experiments). (j) Flow cytometry of $\mathrm{CD}^{+}$lymphocytes in the skin-draining lymph nodes 5 days postinjury. Numbers indicate percent activated $\mathrm{T}_{\text {eff }}$ (upper left) and $\mathrm{T}_{\text {reg }}$ (right) cells, as a percentage of $\mathrm{CD}^{+}$cells. $(\mathbf{k}, \mathbf{l})$ Frequency of $\mathrm{T}_{\text {reg }}(\mathbf{k})$ and $\mathrm{T}_{\text {eff }}(\mathbf{l})$ cells in the SDLNs as a percent of the uninjured SDLN ( $\mathrm{n}=6$ per group; Student's t-test; representative of two experiments). (m) Representative images of Fluoro-gold stained retinas from uninjured or injured eyes. Boxes represent fields counted for retinal ganglion cell quantification. (n) Neuronal survival of mice receiving sham surgery or undergoing deep cervical lymph node removal 2 weeks prior to injury, as assessed by Fluoro-gold staining. Survival is quantified as a percent of control survival $\left(\mathrm{n}=11\right.$ sham and $12 \mathrm{dCLN}$ removed; ${ }^{* *}, \mathrm{p}<$ 0.01 , Student's t-test; representative of two experiments). (o) Retinal ganglion cell counts of the contralateral uninjured retina of mice receiving sham surgery or undergoing deep cervical lymph node removal 2 weeks prior to injury, as assessed by Fluoro-gold staining. RGC counts are quantified as a percent of the control $(n=11$ sham and 12 dCLN removed; Student's t-test; representative of two experiments). 

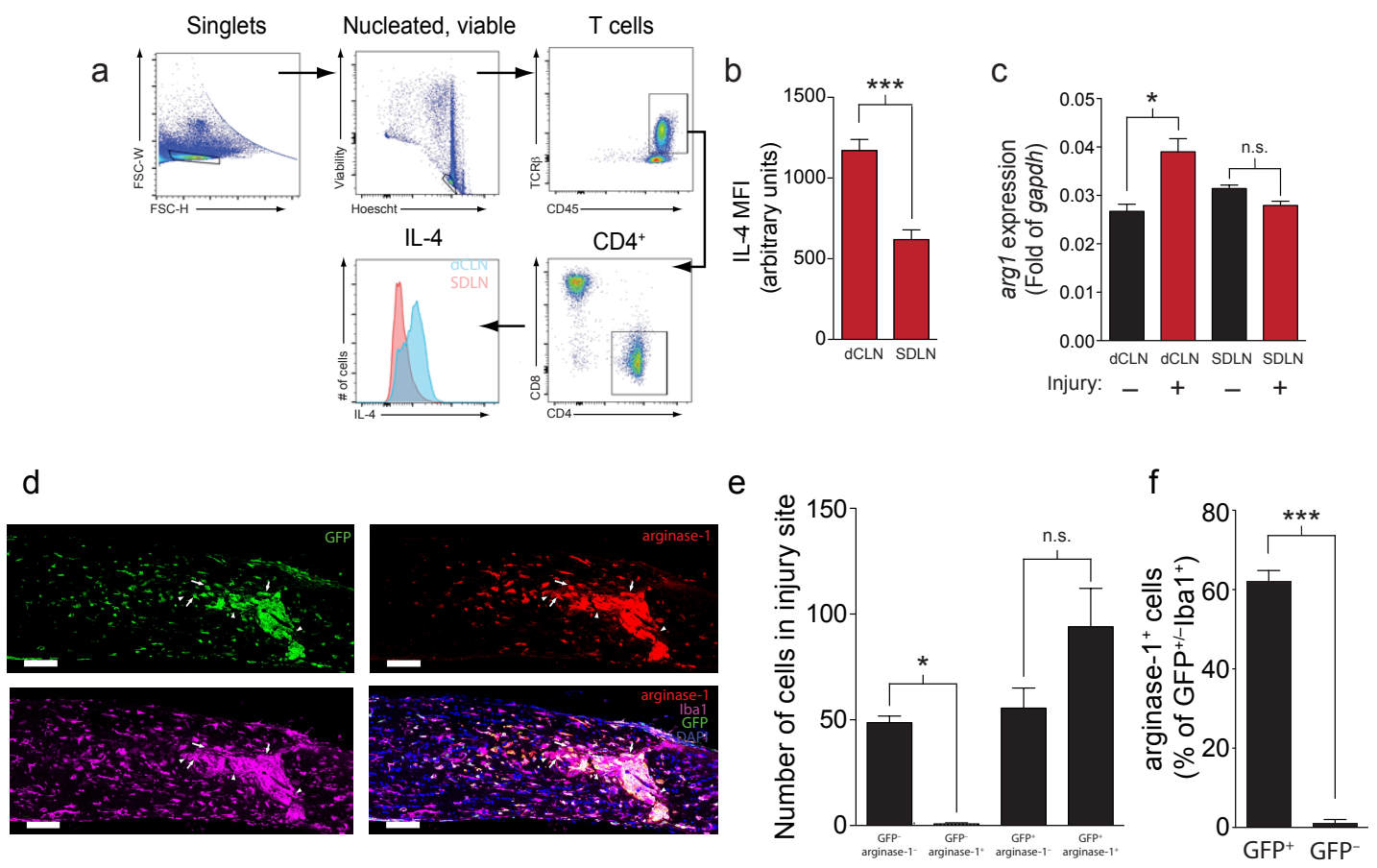

Figure 7: CNS injury promotes a milieu conducive to alternative activation of macrophages in the deep cervical lymph nodes. (a) Gating strategy and representative staining of IL-4 production by $\mathrm{CD}^{+} \mathrm{T}$ cells in the draining deep cervical lymph nodes and skin-draining lymph nodes after CNS injury. (b) Quantification of the mean fluorescence intensity of IL-4 staining of $\mathrm{CD}^{+} \mathrm{T}$ cells in the deep cervical lymph node or skin-draining lymph node $\left(\mathrm{n}=4\right.$ per group; ${ }^{* * *}, \mathrm{p}<0.001$; Student's t-test; representative of two experiments). (c) $\arg 1 \mathrm{mRNA}$ expression of bone-marrow derived macrophages that had been co-cultured with $\mathrm{CD}^{+} \mathrm{T}$ cells from the indicated lymph nodes of mice with or without optic nerve injury for 24 hours (n $=3$ per group; *, $\mathrm{p}<$ 0.05; One-way ANOVA with Bonferroni's post-test; representative of $>3$ experiments). (d) Representative images of injured optic nerves of GFP $\Rightarrow \mathrm{C} 57 \mathrm{~B} 1 / 6$ bone marrow 
chimeras stained for arginase-1 and Ibal. Arrowheads point to $\mathrm{GFP}^{-}$radio-resistant microglia, while arrows point to infiltrating macrophages (scale bar $=100 \mu \mathrm{m}) .(\mathbf{e})$ Quantification of percent of $\mathrm{Ibal}^{+}$cells in the injured optic nerve that are $\mathrm{GFP}^{+}$arginase$1^{+}, \mathrm{GFP}^{+}$arginase- $1^{-}, \mathrm{GFP}^{-}$arginase- $1^{+}$, and $\mathrm{GFP}^{-}$arginase- $1^{-}(\mathrm{n}=3$ per group; One-way ANOVA with Bonferroni's post-test; *, p $<0.05$ ). (f) Quantification of the percent of $\mathrm{GFP}^{+}$and $\mathrm{GFP}^{-}$cells that are arginase- $1^{+}$in the injured optic nerve $(\mathrm{n}=3$ per group; Student's t-test; $\left.{ }^{* * *}, \mathrm{p}<0.001\right)$. 

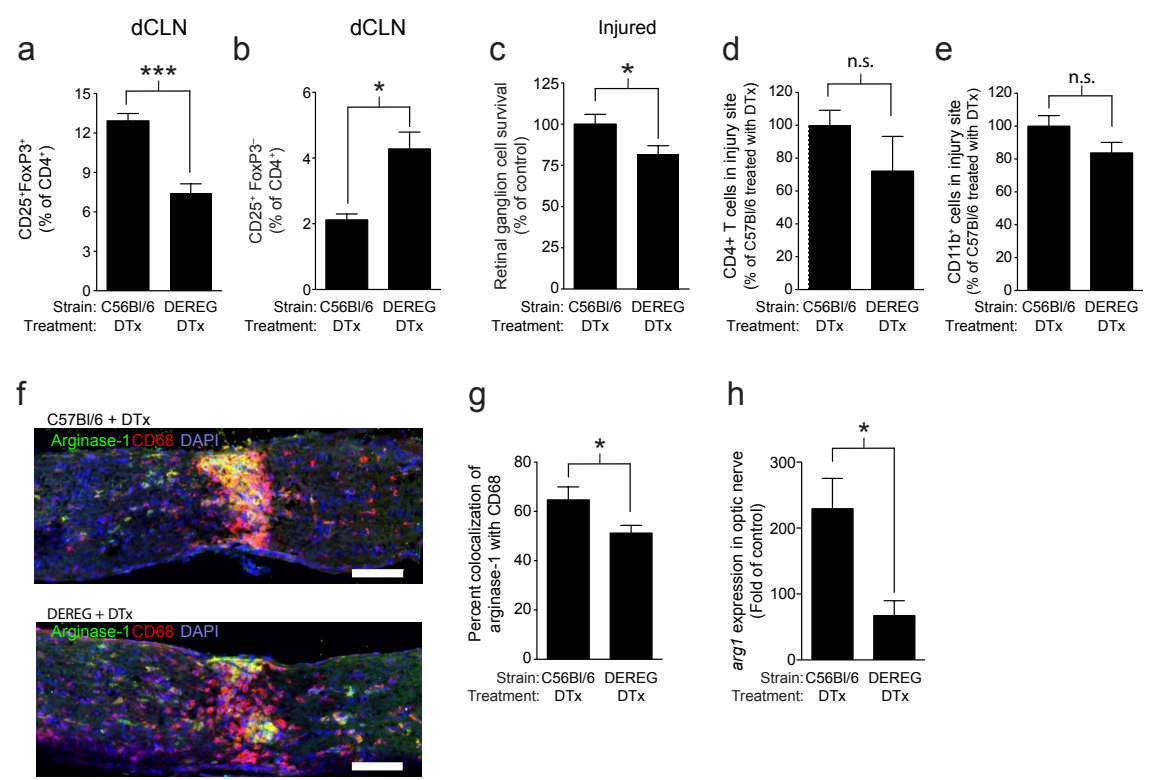

g

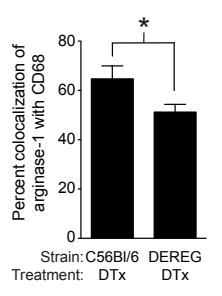

h

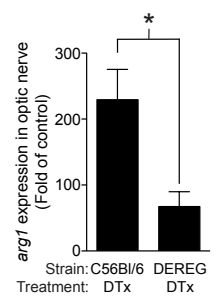

Figure 8: Alleviation of $T_{\text {reg }}$ suppression after CNS injury leads to a reduced neuronal survival after optic nerve injury. (a, b) Bar graphs represent quantification of flow cytometry analysis of the deep cervical lymph nodes of DEREG or wild type littermates treated with DTx two days before injury and on the day of injury, showing percent of CD25 $5^{+}$Foxp $^{+} \mathrm{T}_{\text {reg }}$ cells (a) and of CD $25^{+} \mathrm{Foxp}^{-} \mathrm{T}_{\text {eff }}$ cells (b), graphed as a percentage of $\mathrm{TCR} \beta^{+} \mathrm{CD}^{+}$cells $(\mathrm{n}=12$ wild type and 9 DEREG treated mice; ***, $\mathrm{p}<0.001 ; *, \mathrm{p}<$ 0.05; Student's t-test; representative of three experiments). (c) Neuronal survival after optic nerve injury in DEREG and wild type mice injected with $40 \mu \mathrm{g} / \mathrm{kg}$ DTx two days before injury and on the day of injury. Survival is quantified as a percent of control survival. ( $\mathrm{n}=19$ wild type and 25 DEREG; *, $\mathrm{p}<0.05$, Student's t-test, representative of three experiments). (d) Quantification of the number of $\mathrm{CD}^{+} \mathrm{T}$ cells found in the injury site of DEREG mice treated with DTx normalized to the number of $\mathrm{CD} 4^{+} \mathrm{T}$ cells found in the injury site of $\mathrm{C} 57 \mathrm{Bl} / 6$ mice treated with DTx. $(\mathrm{n}=3$ per group; Student's t-test; 
representative of 2 experiments) (e) Quantification of the number of $\mathrm{CD} 11 \mathrm{~b}^{+}$cells found in the injury site, normalized to the number of $\mathrm{CD} 11 \mathrm{~b}^{+} \mathrm{T}$ cells found in the injury site of C57Bl/6 mice treated with DTx. $(\mathrm{n}=3 \mathrm{C} 57 \mathrm{Bl} / 6$ treated with DTx and 9 DEREG treated with DTx, Student's t-test; representative of 2 experiments) (f) Representative images of CD68 and arginase-1 in injured optic nerve of DEREG and WT mice treated with two doses of $40 \mu \mathrm{g} / \mathrm{kg}$ DTx. (g) Arginase- $1^{+}$area graphed as a percent of $\mathrm{CD}^{+} 8^{+}$area in C57B1/6 or DEREG mice treated with DTx $(n=3$ C57B1/6 treated with DTx and 8 DEREG treated with DTx; *, p < 0.05; Student's t-test). (h) Quantitative PCR for argl of optic nerves of C57Bl/6 or DEREG mice treated with $40 \mu \mathrm{g} / \mathrm{kg}$ DTx 2 days before injury and on the day of injury normalized to argl expression in the contralateral uninjured nerve $(\mathrm{n}=7 \mathrm{C} 57 \mathrm{Bl} / 6$ treated with DTx and 4 DEREG treated with DTx; *, $\mathrm{p}<0.05$; Student's t-test; representative of 2 experiments). 
a

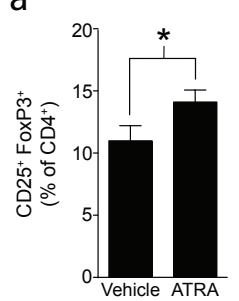

d

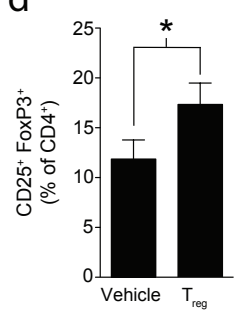

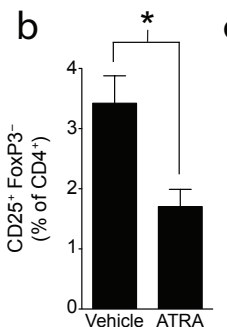

C
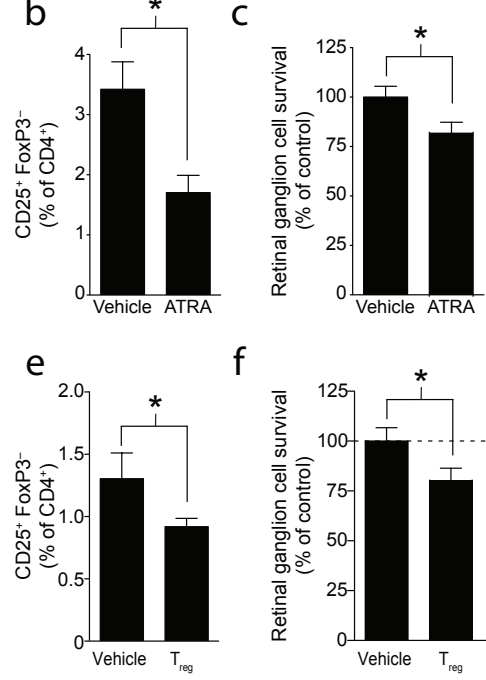

$f$

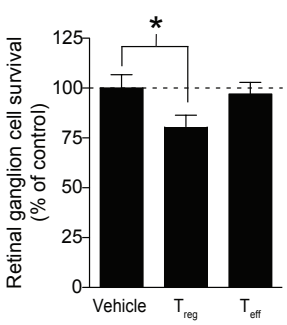

Figure 9: Potentiation of $T_{\text {reg }}$ function impairs neuronal survival after optic nerve injury (a, b) Bar graphs represent quantification of flow cytometry analysis of the deep cervical lymph nodes of wild type mice treated with vehicle or ATRA showing percent of CD2 $5^{+}$Foxp $^{+} \mathrm{T}_{\text {reg }}$ cells (a) and of CD25 $5^{+}$Foxp $^{-} \mathrm{T}_{\text {eff }}$ cells (b), graphed as a percentage of $\mathrm{TCR} \beta^{+} \mathrm{CD} 4^{+}$cells $(\mathrm{n}=7$ vehicle treated and $\mathrm{n}=9$ ATRA treated; * $\mathrm{p}<0.05$, Student's ttest; representative of two experiments (c) Retinal ganglion cell survival in wild type mice treated with vehicle or ATRA. Survival is quantified as a percent of control survival. $\left(\mathrm{n}=7\right.$, vehicle and $\mathrm{n}=9$, ATRA; ${ }^{*}, \mathrm{p}<0.05$, Student's t-test, representative of two experiments). (d, e) Bar graphs represent quantification of flow cytometry analysis of deep cervical lymph nodes of wild type mice treated with vehicle or $1 \times 10^{6}$ exogenous $\mathrm{T}_{\text {reg }}$ cells one day before injury and one day after injury, showing percent of CD25 ${ }^{+}$Foxp $^{+} \mathrm{T}_{\text {reg }}$ cells (d) and of CD25 $5^{+}$Foxp $^{-} \mathrm{T}_{\text {eff }}$ cells (e), graphed as a percentage of $\operatorname{TCR} \beta^{+} \mathrm{CD} 4^{+}$cells $\left(\mathrm{n}=12 \mathrm{~T}_{\text {reg }}\right.$ cells injected and $\mathrm{n}=13$ vehicle injected; ${ }^{*}, \mathrm{p}<0.05$ Student's t-test). (f) Neuronal survival in wild type mice injected with vehicle, $1 \times 10^{6} \mathrm{~T}_{\text {reg }}$ 
or $1 \times 10^{6} \mathrm{~T}_{\text {eff }}$ cells two days before injury and on the day of injury. Survival is quantified as a percent of control survival $\left(n=13\right.$ vehicle injected, $12 T_{\text {reg }}$ cell injected, and $7 T_{\text {eff }}$ cell injected; *, $\mathrm{p}<0.05$, One-way ANOVA with Bonferroni's post-test; representative of two experiments). 

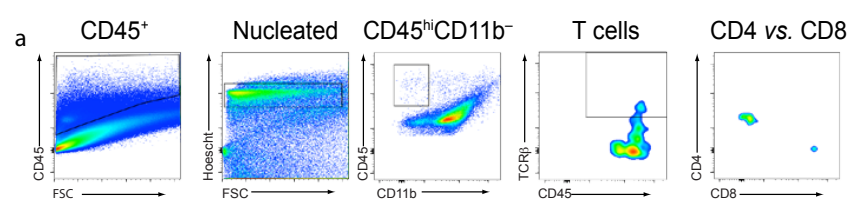

b
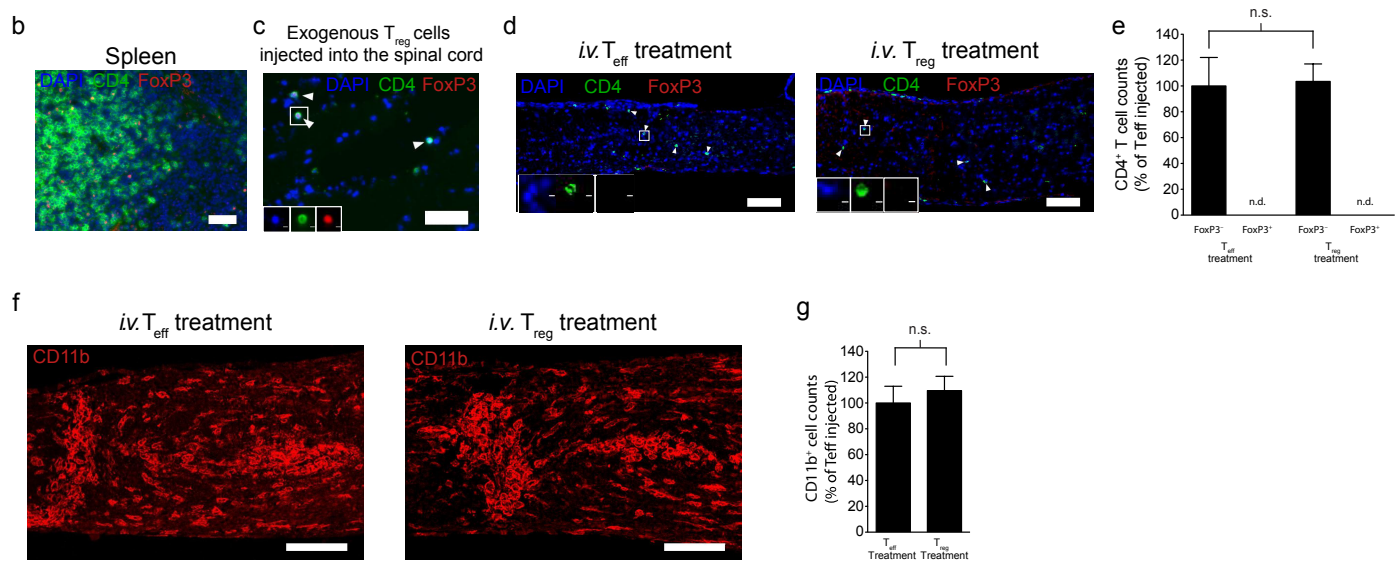

Figure 10: Boost with exogenous $T_{\text {reg }}$ cells does not alter immune cell infiltration into the injury site. (a) Representative gates of flow cytometry of $\mathrm{CD}^{+}$and $\mathrm{CD} 8^{+}$lymphocytes in the injured optic nerve seven days post-injury. Optic nerves were pooled from eight mice, and cells were stained for analysis by flow cytometry. (b) Representative image from splenic tissue stained for CD4 (green) and Foxp3 (red) (scale bar $=100 \mu \mathrm{m})$. (c) Representative image from spinal cord tissue directly injected (ex vivo) with in vitro induced regulatory T cells stained for CD4 (green) and Foxp3 (red) (scale bar $=50 \mu \mathrm{m})$. (d) Representative images of $\mathrm{CD} 4^{+} \mathrm{Foxp}^{-}$and $\mathrm{CD} 4^{+} \mathrm{Foxp}^{+} \mathrm{T}$ cells in the optic nerve parenchyma of $\mathrm{T}_{\text {eff- }}$ and $\mathrm{T}_{\text {reg-treated mice }}($ scale bar $=100 \mu \mathrm{m})$. (e) Quantification of the number of $\mathrm{CD} 4^{+} \mathrm{Foxp}^{-}$and $\mathrm{CD} 4^{+} \mathrm{Foxp}^{+} \mathrm{T}$ cells in the optic nerve parenchyma of $\mathrm{T}_{\text {eff }}$ and $T_{\text {reg }}$ treated mice $(n=4$ mice per group; One-way ANOVA with Bonferroni's posttest; scale bar $=100 \mu \mathrm{m})$. (f) Representative images of $\mathrm{CD} 11 \mathrm{~b}^{+}$cells in injury site of the optic nerve of $\mathrm{T}_{\text {eff- }}$ and $\mathrm{T}_{\text {reg-treated mice }}$ (scale bar $\left.=100 \mu \mathrm{m}\right)$. $(\mathrm{g})$ Quantification of the 
number of $C D 11 b^{+}$cells in injury site of the optic nerve of $T_{\text {eff }}$ and $T_{\text {reg }}$ treated mice $(n=$ 9 mice per group; Student's t-test; representative of two experiments). 
a

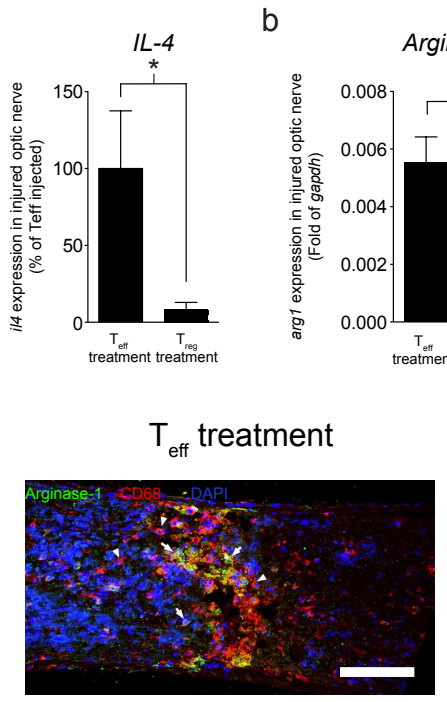

Arginase-1

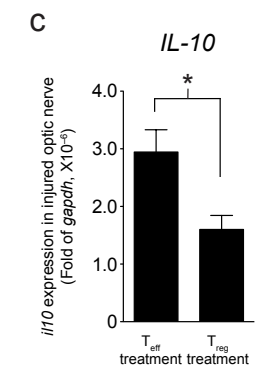

$T_{\text {reg }}$ treatment

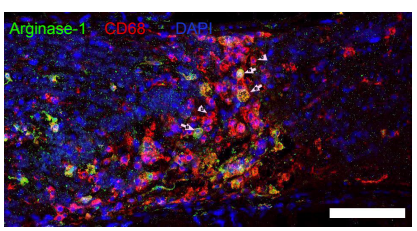

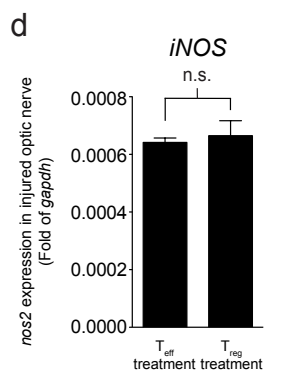

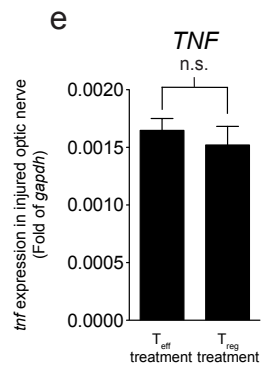

g

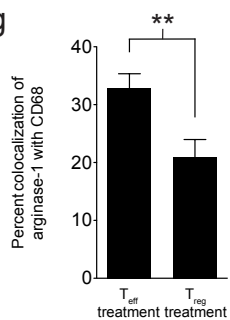

Figure 11: $T_{\text {reg }}$ cell injection leads to a loss of an alternative activation phenotype of myeloid cells at the site of injury. Optic nerves of $\mathrm{T}_{\text {eff- }}$ and $\mathrm{T}_{\text {reg- }}$ cell injected mice were collected 7 days post injury and examined for expression of the following genes relative to expression of gapdh (a) il4 (n $=11$ per group *, $\mathrm{p}<0.05$; Student's t-test; representative of two experiments); (b) $\arg 1 ;$ (c) illo; (d) nos2; (e) thf; (b-e; $\mathrm{n}=6$ per group; *, $\mathrm{p}<0.05$; Student's t-test); (f) Representative images of $\mathrm{T}_{\text {eff- }}$ and $\mathrm{T}_{\text {reg- }}$ cell injected mice 7 days after injury stained for arginase-1 (green) and CD68 (red) (scale bar $=100 \mu \mathrm{m}) .(\mathrm{g})$ Arginase- $1^{+}$area graphed as a percent of CD68 $8^{+}$area in $\mathrm{T}_{\text {eff- }}$ and $\mathrm{T}_{\text {reg- }}$ cell injected mice 7 days post-injury $\left(n=9 T_{\text {eff }}\right.$ cell injected and $6 T_{\text {reg }}$ cell injected; ${ }^{* *}, p<$ 0.01; Student's t-test; representative of two experiments). 


\section{Appendix I: References}

Albrecht, P.J., Dahl, J.P., Stoltzfus, O.K., Levenson, R., and Levison, S.W. (2002). Ciliary neurotrophic factor activates spinal cord astrocytes, stimulating their production and release of fibroblast growth factor-2, to increase motor neuron survival. Exp Neurol 173, 46-62.

Allan, S.M., Tyrrell, P.J., and Rothwell, N.J. (2005). Interleukin-1 and neuronal injury. Nat Rev Immunol 5, 629-640.

Ankeny, D.P., Guan, Z., and Popovich, P.G. (2009). B cells produce pathogenic antibodies and impair recovery after spinal cord injury in mice. J Clin Invest 119, 2990-2999.

Ankeny, D.P., Lucin, K.M., Sanders, V.M., McGaughy, V.M., and Popovich, P.G. (2006). Spinal cord injury triggers systemic autoimmunity: evidence for chronic B lymphocyte activation and lupus-like autoantibody synthesis. J Neurochem 99, 1073-1087.

Anthony, R.M., Urban, J.F., Jr., Alem, F., Hamed, H.A., Rozo, C.T., Boucher, J.L., Van Rooijen, N., and Gause, W.C. (2006). Memory T(H)2 cells induce alternatively activated macrophages to mediate protection against nematode parasites. Nat Med 12, 955-960.

Araque, A., Parpura, V., Sanzgiri, R.P., and Haydon, P.G. (1999). Tripartite synapses: glia, the unacknowledged partner. Trends Neurosci 22, 208-215.

Archambault, A.S., Sim, J., Gimenez, M.A., and Russell, J.H. (2005). Defining antigendependent stages of $\mathrm{T}$ cell migration from the blood to the central nervous system parenchyma. Eur J Immunol 35, 1076-1085.

Bachis, A., Colangelo, A.M., Vicini, S., Doe, P.P., De Bernardi, M.A., Brooker, G., and Mocchetti, I. (2001). Interleukin-10 prevents glutamate-mediated cerebellar granule cell death by blocking caspase-3-like activity. J Neurosci 21, 3104-3112.

Bao, F., Bailey, C.S., Gurr, K.R., Bailey, S.I., Rosas-Arellano, M.P., Dekaban, G.A., and Weaver, L.C. (2009). Increased oxidative activity in human blood neutrophils and monocytes after spinal cord injury. Exp Neurol 215, 308-316.

Baruch, K., Ron-Harel, N., Gal, H., Deczkowska, A., Shifrut, E., Ndifon, W., MirlasNeisberg, N., Cardon, M., Vaknin, I., Cahalon, L., et al. (2013). CNS-specific immunity at the choroid plexus shifts toward destructive Th2 inflammation in brain aging. Proc Natl Acad Sci U S A 110, 2264-2269. 
Basso, D.M., Fisher, L.C., Anderson, A.J., Jakeman, L.B., McTigue, D.M., and Popovich, P.G. (2006). Basso Mouse Scale for locomotion detects differences in recovery after spinal. J Neurotrauma 23, 635-659.

Becerra, J.L., Puckett, W.R., Hiester, E.D., Quencer, R.M., Marcillo, A.E., Post, M.J., and Bunge, R.P. (1995). MR-pathologic comparisons of wallerian degeneration in spinal cord injury. AJNR Am J Neuroradiol 16, 125-133.

Ben-Sasson, S.Z., Caucheteux, S., Crank, M., Hu-Li, J., and Paul, W.E. (2011). IL-1 acts on $\mathrm{T}$ cells to enhance the magnitude of in vivo immune responses. Cytokine 56, 122125.

Bennett, M.H., Trytko, B., and Jonker, B. (2012). Hyperbaric oxygen therapy for the adjunctive treatment of traumatic brain injury. Cochrane Database Syst Rev 12, CD004609.

Benz, C.C., and Yau, C. (2008). Ageing, oxidative stress and cancer: paradigms in parallax. Nat Rev Cancer 8, 875-879.

Bernardinelli, Y., Magistretti, P.J., and Chatton, J.Y. (2004). Astrocytes generate Na+mediated metabolic waves. Proc Natl Acad Sci U S A 101, 14937-14942.

Bernhard, M., Gries, A., Kremer, P., and Böttiger, B.W. (2005). Spinal cord injury (SCI)--prehospital management. Resuscitation 66, 127-139.

Beschorner, R., Dietz, K., Schauer, N., Mittelbronn, M., Schluesener, H.J., Trautmann, K., Meyermann, R., and Simon, P. (2007). Expression of EAAT1 reflects a possible neuroprotective function of reactive astrocytes and activated microglia following human traumatic brain injury. Histol Histopathol 22, 515-526.

Bethea, J.R., Nagashima, H., Acosta, M.C., Briceno, C., Gomez, F., Marcillo, A.E., Loor, K., Green, J., and Dietrich, W.D. (1999). Systemically administered interleukin-10 reduces tumor necrosis factor-alpha production and significantly improves functional recovery following traumatic spinal cord injury in rats. J Neurotrauma 16, 851-863.

Bettelli, E., Baeten, D., Jäger, A., Sobel, R.A., and Kuchroo, V.K. (2006a). Myelin oligodendrocyte glycoprotein-specific T and B cells cooperate to induce a Devic-like disease in mice. J Clin Invest 116, 2393-2402.

Bettelli, E., Carrier, Y., Gao, W., Korn, T., Strom, T.B., Oukka, M., Weiner, H.L., and Kuchroo, V.K. (2006b). Reciprocal developmental pathways for the generation of pathogenic effector TH17 and regulatory T cells. Nature 441, 235-238.

Bialas, A.R., and Stevens, B. (2013). TGF- $\beta$ signaling regulates neuronal C1q expression and developmental synaptic refinement. Nat Neurosci 16, 1773-1782.

Blaeser, F., Bryce, P.J., Ho, N., Raman, V., Dedeoglu, F., Donaldson, D.D., Geha, R.S., Oettgen, H.C., and Chatila, T.A. (2003). Targeted inactivation of the IL-4 receptor alpha chain I4R motif promotes allergic airway inflammation. J Exp Med 198, 11891200.

Boato, F., Rosenberger, K., Nelissen, S., Geboes, L., Peters, E.M., Nitsch, R., and Hendrix, S. (2013). Absence of IL-1 $\beta$ positively affects neurological outcome, lesion development and axonal plasticity after spinal cord injury. J Neuroinflammation 10, 6. 
Bonneh-Barkay, D., and Wiley, C.A. (2009). Brain extracellular matrix in neurodegeneration. Brain Pathol 19, 573-585.

Buch, T., Polic, B., Clausen, B.E., Weiss, S., Akilli-Ozturk, O., Chang, C.H., Flavell, R., Schulz, A., Jonjic, S., Waisman, A., and Forster, I. (2006). MHC class II expression through a hitherto unknown pathway supports $\mathrm{T}$ helper cell-dependent immune responses: implications for MHC class II deficiency. Blood 107, 1434-1444.

Bull, N.D., Irvine, K.A., Franklin, R.J., and Martin, K.R. (2009). Transplanted oligodendrocyte precursor cells reduce neurodegeneration in a model of glaucoma. Invest Ophthalmol Vis Sci 50, 4244-4253.

Burns, K., Martinon, F., Esslinger, C., Pahl, H., Schneider, P., Bodmer, J.L., Di Marco, F., French, L., and Tschopp, J. (1998). MyD88, an adapter protein involved in interleukin-1 signaling. J Biol Chem 273, 12203-12209.

Busch, S.A., Horn, K.P., Cuascut, F.X., Hawthorne, A.L., Bai, L., Miller, R.H., and Silver, J. (2010). Adult NG2+ cells are permissive to neurite outgrowth and stabilize sensory axons during macrophage-induced axonal dieback after spinal cord injury. J Neurosci 30, 255-265.

Bush, T.G., Puvanachandra, N., Horner, C.H., Polito, A., Ostenfeld, T., Svendsen, C.N., Mucke, L., Johnson, M.H., and Sofroniew, M.V. (1999). Leukocyte infiltration, neuronal degeneration, and neurite outgrowth after ablation of scar-forming, reactive astrocytes in adult transgenic mice. Neuron 23, 297-308.

Buss, A., Brook, G.A., Kakulas, B., Martin, D., Franzen, R., Schoenen, J., Noth, J., and Schmitt, A.B. (2004). Gradual loss of myelin and formation of an astrocytic scar during Wallerian degeneration in the human spinal cord. Brain 127, 34-44.

Butovsky, O., Jedrychowski, M.P., Moore, C.S., Cialic, R., Lanser, A.J., Gabriely, G., Koeglsperger, T., Dake, B., Wu, P.M., Doykan, C.E., et al. (2013). Identification of a unique TGF- $\beta$-dependent molecular and functional signature in microglia. Nat Neurosci.

Byram, S.C., Carson, M.J., DeBoy, C.A., Serpe, C.J., Sanders, V.M., and Jones, K.J. (2004). CD4-positive $\mathrm{T}$ cell-mediated neuroprotection requires dual compartment antigen presentation. J Neurosci 24, 4333-4339.

Carlson, N.G., Wieggel, W.A., Chen, J., Bacchi, A., Rogers, S.W., and Gahring, L.C. (1999). Inflammatory cytokines IL-1 alpha, IL-1 beta, IL-6, and TNF-alpha impart neuroprotection to an excitotoxin through distinct pathways. J Immunol 163, 39633968.

Center, N.S.C.I.S. (2013). Spinal cord injury facts and figures at a glance. J Spinal Cord Med 36, 1-2.

Chang, G.H., Barbaro, N.M., and Pieper, R.O. (2000). Phosphatidylserine-dependent phagocytosis of apoptotic glioma cells by normal human microglia, astrocytes, and glioma cells. Neuro Oncol 2, 174-183.

Chen, K.B., Uchida, K., Nakajima, H., Yayama, T., Hirai, T., Rodriguez Guerrero, A., Kobayashi, S., Ma, W.Y., Liu, S.Y., Zhu, P., and Baba, H. (2011). High-mobility group box-1 and its receptors contribute to proinflammatory response in the acute phase of spinal cord injury in rats. Spine (Phila Pa 1976) 36, 2122-2129. 
Chen, M.S., Huber, A.B., van der Haar, M.E., Frank, M., Schnell, L., Spillmann, A.A., Christ, F., and Schwab, M.E. (2000). Nogo-A is a myelin-associated neurite outgrowth inhibitor and an antigen for monoclonal antibody IN-1. Nature 403, 434-439.

Chen, Z.J., Ughrin, Y., and Levine, J.M. (2002). Inhibition of axon growth by oligodendrocyte precursor cells. Mol Cell Neurosci 20, 125-139.

Cheng, G., Kong, R.H., Zhang, L.M., and Zhang, J.N. (2012). Mitochondria in traumatic brain injury and mitochondrial-targeted multipotential therapeutic strategies. $\mathrm{Br} \mathrm{J}$ Pharmacol 167, 699-719.

Christopherson, K.S., Ullian, E.M., Stokes, C.C., Mullowney, C.E., Hell, J.W., Agah, A., Lawler, J., Mosher, D.F., Bornstein, P., and Barres, B.A. (2005). Thrombospondins are astrocyte-secreted proteins that promote CNS synaptogenesis. Cell 120, 421-433.

Chung, Y., Chang, S.H., Martinez, G.J., Yang, X.O., Nurieva, R., Kang, H.S., Ma, L., Watowich, S.S., Jetten, A.M., Tian, Q., and Dong, C. (2009). Critical regulation of early Th17 cell differentiation by interleukin-1 signaling. Immunity 30, 576-587.

Coleman, M. (2005). Axon degeneration mechanisms: commonality amid diversity. Nat Rev Neurosci 6, 889-898.

d'Hennezel, E., Yurchenko, E., Sgouroudis, E., Hay, V., and Piccirillo, C.A. (2011). Single-cell analysis of the human $\mathrm{T}$ regulatory population uncovers functional heterogeneity and instability within FOXP3+ cells. J Immunol 186, 6788-6797.

D'Mello, C., Le, T., and Swain, M.G. (2009). Cerebral microglia recruit monocytes into the brain in response to tumor necrosis factoralpha signaling during peripheral organ inflammation. J Neurosci 29, 2089-2102.

Davalos, D., Grutzendler, J., Yang, G., Kim, J.V., Zuo, Y., Jung, S., Littman, D.R., Dustin, M.L., and Gan, W.B. (2005). ATP mediates rapid microglial response to local brain injury in vivo. Nat Neurosci 8, 752-758.

David, S., and Kroner, A. (2011). Repertoire of microglial and macrophage responses after spinal cord injury. Nat Rev Neurosci 12, 388-399.

de Rivero Vaccari, J.P., Lotocki, G., Marcillo, A.E., Dietrich, W.D., and Keane, R.W. (2008). A molecular platform in neurons regulates inflammation after spinal cord injury. J Neurosci 28, 3404-3414.

Degryse, B., Bonaldi, T., Scaffidi, P., Müller, S., Resnati, M., Sanvito, F., Arrigoni, G., and Bianchi, M.E. (2001). The high mobility group (HMG) boxes of the nuclear protein HMG1 induce chemotaxis and cytoskeleton reorganization in rat smooth muscle cells. J Cell Biol 152, 1197-1206.

Deng, W., Aimone, J.B., and Gage, F.H. (2010). New neurons and new memories: how does adult hippocampal neurogenesis affect learning and memory? Nat Rev Neurosci 11, 339-350.

Deppmann, C.D., Mihalas, S., Sharma, N., Lonze, B.E., Niebur, E., and Ginty, D.D. (2008). A model for neuronal competition during development. Science 320, 369373.

Derecki, N.C., Cardani, A.N., Yang, C.H., Quinnies, K.M., Crihfield, A., Lynch, K.R., and Kipnis, J. (2010). Regulation of learning and memory by meningeal immunity: a key role for IL-4. J Exp Med 207, 1067-1080. 
Derecki, N.C., Cronk, J.C., Lu, Z., Xu, E., Abbott, S.B., Guyenet, P.G., and Kipnis, J. (2012). Wild-type microglia arrest pathology in a mouse model of Rett syndrome. Nature 484, 105-109.

DeVivo, M.J. (1997). Causes and costs of spinal cord injury in the United States. Spinal Cord 35, 809-813.

Dickendesher, T.L., Baldwin, K.T., Mironova, Y.A., Koriyama, Y., Raiker, S.J., Askew, K.L., Wood, A., Geoffroy, C.G., Zheng, B., Liepmann, C.D., et al. (2012). NgR1 and NgR3 are receptors for chondroitin sulfate proteoglycans. Nat Neurosci 15, 703-712.

Ding, Z., Liu, S., Wang, X., Khaidakov, M., Dai, Y., and Mehta, J.L. (2013). Oxidant stress in mitochondrial DNA damage, autophagy and inflammation in atherosclerosis. Sci Rep 3, 1077.

Dunai, Z., Bauer, P.I., and Mihalik, R. (2011). Necroptosis: biochemical, physiological and pathological aspects. Pathol Oncol Res 17, 791-800.

Dunn, S.L., Young, E.A., Hall, M.D., and McNulty, S. (2002). Activation of astrocyte intracellular signaling pathways by interleukin-1 in rat primary striatal cultures. Glia 37, 31-42.

Dupré-Crochet, S., Erard, M., and Nüße, O. (2013). ROS production in phagocytes: why, when, and where? J Leukoc Biol 94, 657-670.

Durack, D.T., Sumi, S.M., and Klebanoff, S.J. (1979). Neurotoxicity of human eosinophils. Proc Natl Acad Sci U S A 76, 1443-1447.

Eddleman, C.S., Ballinger, M.L., Smyers, M.E., Fishman, H.M., and Bittner, G.D. (1998). Endocytotic formation of vesicles and other membranous structures induced by Ca2+ and axolemmal injury. J Neurosci 18, 4029-4041.

Elliott, M.R., Chekeni, F.B., Trampont, P.C., Lazarowski, E.R., Kadl, A., Walk, S.F., Park, D., Woodson, R.I., Ostankovich, M., Sharma, P., et al. (2009). Nucleotides released by apoptotic cells act as a find-me signal to promote phagocytic clearance. Nature 461, 282-286.

Erez, H., and Spira, M.E. (2008). Local self-assembly mechanisms underlie the differential transformation of the proximal and distal cut axonal ends into functional and aberrant growth cones. J Comp Neurol 507, 1019-1030.

Evans, T.A., Barkauskas, D.S., Myers, J.T., Hare, E.G., You, J.Q., Ransohoff, R.M., Huang, A.Y., and Silver, J. (2014). High-resolution intravital imaging reveals that bloodderived macrophages but not resident microglia facilitate secondary axonal dieback in traumatic spinal cord injury. Exp Neurol 254, 109-120.

Falcone, M., and Bloom, B.R. (1997). A T helper cell 2 (Th2) immune response against non-self antigens modifies the cytokine profile of autoimmune $\mathrm{T}$ cells and protects against experimental allergic encephalomyelitis. J Exp Med 185, 901-907.

Farkas, 0., Lifshitz, J., and Povlishock, J.T. (2006). Mechanoporation induced by diffuse traumatic brain injury: an irreversible or reversible response to injury? J Neurosci 26, 3130-3140.

Fenn, A.M., Hall, J.C., Gensel, J.C., Popovich, P.G., and Godbout, J.P. (2014). IL-4 Signaling Drives a Unique Arginase+/IL-1 $\beta+$ Microglia Phenotype and Recruits Macrophages to the Inflammatory CNS: Consequences of Age-Related Deficits in IL$4 \mathrm{R} \alpha$ after Traumatic Spinal Cord Injury. J Neurosci 34, 8904-8917. 
Filosa, J.A., and Iddings, J.A. (2013). Astrocyte regulation of cerebral vascular tone. Am J Physiol Heart Circ Physiol 305, H609-619.

Finkelman, F.D., Katona, I.M., Urban, J.F., Holmes, J., Ohara, J., Tung, A.S., Sample, J.V., and Paul, W.E. (1988). IL-4 is required to generate and sustain in vivo IgE responses. J Immunol 141, 2335-2341.

Fitch, M.T., and Silver, J. (2008). CNS injury, glial scars, and inflammation: Inhibitory extracellular matrices and regeneration failure. Exp Neurol 209, 294-301.

Fontenot, J.D., Gavin, M.A., and Rudensky, A.Y. (2003). Foxp3 programs the development and function of CD4+CD25+ regulatory T cells. Nat Immunol 4, 330336.

Frank, M., and Wolburg, H. (1996). Cellular reactions at the lesion site after crushing of the rat optic nerve. Glia 16, 227-240.

Franke, H., and Illes, P. (2006). Involvement of P2 receptors in the growth and survival of neurons in the CNS. Pharmacol Ther 109, 297-324.

Franke, H., Verkhratsky, A., Burnstock, G., and Illes, P. (2012). Pathophysiology of astroglial purinergic signalling. Purinergic Signal 8, 629-657.

Frenkel, D., Huang, Z., Maron, R., Koldzic, D.N., Moskowitz, M.A., and Weiner, H.L. (2005). Neuroprotection by IL-10-producing MOG CD4+ T cells following ischemic stroke. J Neurol Sci 233, 125-132.

Fricker, M., Neher, J.J., Zhao, J.W., Théry, C., Tolkovsky, A.M., and Brown, G.C. (2012). MFG-E8 mediates primary phagocytosis of viable neurons during neuroinflammation. J Neurosci 32, 2657-2666.

Garg, S.K., Banerjee, R., and Kipnis, J. (2008). Neuroprotective immunity: T cellderived glutamate endows astrocytes with a neuroprotective phenotype. J Immunol 180, 3866-3873.

Garg, S.K., Kipnis, J., and Banerjee, R. (2009). IFN-gamma and IL-4 differentially shape metabolic responses and neuroprotective phenotype of astrocytes. J Neurochem 108, 1155-1166.

Gaudet, A.D., Popovich, P.G., and Ramer, M.S. (2011). Wallerian degeneration: gaining perspective on inflammatory events after peripheral nerve injury. J Neuroinflammation 8, 110.

Gavin, M.A., Clarke, S.R., Negrou, E., Gallegos, A., and Rudensky, A. (2002). Homeostasis and anergy of CD4(+)CD25(+) suppressor T cells in vivo. Nat Immunol 3, 33-41.

Gershon, R.K., Cohen, P., Hencin, R., and Liebhaber, S.A. (1972). Suppressor T cells. J Immunol 108, 586-590.

Ghosh-Roy, A., Wu, Z., Goncharov, A., Jin, Y., and Chisholm, A.D. (2010). Calcium and cyclic AMP promote axonal regeneration in Caenorhabditis elegans and require DLK-1 kinase. J Neurosci 30, 3175-3183.

Girard, S., Brough, D., Lopez-Castejon, G., Giles, J., Rothwell, N.J., and Allan, S.M. (2013). Microglia and macrophages differentially modulate cell death after brain injury caused by oxygen-glucose deprivation in organotypic brain slices. Glia 61, 813-824. 
Gitik, M., Liraz-Zaltsman, S., Oldenborg, P.A., Reichert, F., and Rotshenker, S. (2011). Myelin down-regulates myelin phagocytosis by microglia and macrophages through interactions between CD47 on myelin and SIRP $\alpha$ (signal regulatory protein- $\alpha$ ) on phagocytes. J Neuroinflammation 8, 24.

Glezer, I., Lapointe, A., and Rivest, S. (2006). Innate immunity triggers oligodendrocyte progenitor reactivity and confines damages to brain injuries. FASEB J 20, 750-752.

Gordon, S., and Martinez, F.O. (2010). Alternative activation of macrophages: mechanism and functions. Immunity 32, 593-604.

Gorio, A., Madaschi, L., Zadra, G., Marfia, G., Cavalieri, B., Bertini, R., and Di Giulio, A.M. (2007). Reparixin, an inhibitor of CXCR2 function, attenuates inflammatory responses and promotes recovery of function after traumatic lesion to the spinal cord. J Pharmacol Exp Ther 322, 973-981.

Greenhalgh, A.D., and David, S. (2014). Differences in the phagocytic response of microglia and peripheral macrophages after spinal cord injury and its effects on cell death. J Neurosci 34, 6316-6322.

Griffiths, I., Klugmann, M., Anderson, T., Yool, D., Thomson, C., Schwab, M.H., Schneider, A., Zimmermann, F., McCulloch, M., Nadon, N., and Nave, K.A. (1998). Axonal swellings and degeneration in mice lacking the major proteolipid of myelin. Science 280, 1610-1613.

Gu, X.J., Xu, J., Ma, B.Y., Chen, G., Gu, P.Y., Wei, D., and Hu, W.X. (2014). Effect of glycyrrhizin on traumatic brain injury in rats and its mechanism. Chin J Traumatol 17, 1-7.

Guo, J.D., Li, L., Shi, Y.M., Wang, H.D., Yuan, Y.L., Shi, X.X., and Hou, S.X. (2014). Genetic ablation of receptor for advanced glycation end products promotes functional recovery in mouse model of spinal cord injury. Mol Cell Biochem 390, 215-223.

Hafner-Bratkovič, I., Benčina, M., Fitzgerald, K.A., Golenbock, D., and Jerala, R. (2012). NLRP3 inflammasome activation in macrophage cell lines by prion protein fibrils as the source of IL-1 $\beta$ and neuronal toxicity. Cell Mol Life Sci 69, 4215-4228.

Hamada, Y., Ikata, T., Katoh, S., Katoh, K., Niwa, M., Tsutsumishita, Y., and Fukuzawa, K. (1996). Effects of exogenous transforming growth factor-beta 1 on spinal cord injury in rats. Neurosci Lett 203, 97-100.

Hauben, E., Butovsky, O., Nevo, U., Yoles, E., Moalem, G., Agranov, E., Mor, F., Leibowitz-Amit, R., Pevsner, E., Akselrod, S., et al. (2000). Passive or active immunization with myelin basic protein promotes recovery from spinal cord contusion. Journal of Neuroscience 20, 6421-6430.

Haviv, R., and Stein, R. (1998). The intracellular domain of p55 tumor necrosis factor receptor induces apoptosis which requires different caspases in naive and neuronal PC12 cells. J Neurosci Res 52, 380-389.

Hechler, D., Boato, F., Nitsch, R., and Hendrix, S. (2010). Differential regulation of axon outgrowth and reinnervation by neurotrophin-3 and neurotrophin- 4 in the hippocampal formation. Exp Brain Res 205, 215-221.

Hendrix, S., Kramer, P., Pehl, D., Warnke, K., Boato, F., Nelissen, S., Lemmens, E., Pejler, G., Metz, M., Siebenhaar, F., and Maurer, M. (2013). Mast cells protect from 
post-traumatic brain inflammation by the mast cell-specific chymase mouse mast cell protease-4. FASEB J 27, 920-929.

Hendrix, S., and Nitsch, R. (2007). The role of T helper cells in neuroprotection and regeneration. J Neuroimmunol 184, 100-112.

Herrmann, J.E., Imura, T., Song, B., Qi, J., Ao, Y., Nguyen, T.K., Korsak, R.A., Takeda, K., Akira, S., and Sofroniew, M.V. (2008). STAT3 is a critical regulator of astrogliosis and scar formation after spinal cord injury. J Neurosci 28, 7231-7243.

Herx, L.M., Rivest, S., and Yong, V.W. (2000). Central nervous system-initiated inflammation and neurotrophism in trauma: IL-1 beta is required for the production of ciliary neurotrophic factor. J Immunol 165, 2232-2239.

Herx, L.M., and Yong, V.W. (2001). Interleukin-1 beta is required for the early evolution of reactive astrogliosis following CNS lesion. J Neuropathol Exp Neurol 60, 961-971.

Hirschberg, D.L., Moalem, G., He, J., Mor, F., Cohen, I.R., and Schwartz, M. (1998). Accumulation of passively transferred primed T cells independently of their antigen specificity following central nervous system trauma. J Neuroimmunol 89, 88-96.

Hirschberg, D.L., and Schwartz, M. (1995). Macrophage recruitment to acutely injured central nervous system is inhibited by a resident factor: a basis for an immune-brain barrier. J Neuroimmunol 61, 89-96.

Hori, O., Brett, J., Slattery, T., Cao, R., Zhang, J., Chen, J.X., Nagashima, M., Lundh, E.R., Vijay, S., and Nitecki, D. (1995). The receptor for advanced glycation end products (RAGE) is a cellular binding site for amphoterin. Mediation of neurite outgrowth and co-expression of rage and amphoterin in the developing nervous system. J Biol Chem 270, 25752-25761.

Hori, S., Nomura, T., and Sakaguchi, S. (2003). Control of regulatory T cell development by the transcription factor Foxp3. Science 299, 1057-1061.

Horn, K.P., Busch, S.A., Hawthorne, A.L., van Rooijen, N., and Silver, J. (2008). Another barrier to regeneration in the CNS: activated macrophages induce extensive retraction of dystrophic axons through direct physical interactions. J Neurosci 28, 9330-9341.

Horvath, G.L., Schrum, J.E., De Nardo, C.M., and Latz, E. (2011). Intracellular sensing of microbes and danger signals by the inflammasomes. Immunol Rev 243, 119-135.

Hudson, C.A., Christophi, G.P., Gruber, R.C., Wilmore, J.R., Lawrence, D.A., and Massa, P.T. (2008). Induction of IL-33 expression and activity in central nervous system glia. J Leukoc Biol 84, 631-643.

Humphreys, N.E., and Grencis, R.K. (2009). IL-1-dependent, IL-1R1-independent resistance to gastrointestinal nematodes. Eur J Immunol 39, 1036-1045.

Humphreys, N.E., Xu, D., Hepworth, M.R., Liew, F.Y., and Grencis, R.K. (2008). IL-33, a potent inducer of adaptive immunity to intestinal nematodes. J Immunol 180, 24432449.

Iyer, S.S., and Cheng, G. (2012). Role of interleukin 10 transcriptional regulation in inflammation and autoimmune disease. Crit Rev Immunol 32, 23-63.

Jiang, H.R., Milovanović, M., Allan, D., Niedbala, W., Besnard, A.G., Fukada, S.Y., AlvesFilho, J.C., Togbe, D., Goodyear, C.S., Linington, C., et al. (2012). IL-33 attenuates EAE 
by suppressing IL-17 and IFN- $\gamma$ production and inducing alternatively activated macrophages. Eur J Immunol 42, 1804-1814.

Johnson, B.D., Jing, W., and Orentas, R.J. (2007a). CD25+ regulatory T cell inhibition enhances vaccine-induced immunity to neuroblastoma. J Immunother 30, 203-214. Johnson, T.V., Camras, C.B., and Kipnis, J. (2007b). Bacterial DNA confers neuroprotection after optic nerve injury by suppressing CD4(+) CD25(+) regulatory T-Cell activity. Investigative Ophthalmology \& Visual Science 48, 3441-3449.

Jones, T.B., Ankeny, D.P., Guan, Z., McGaughy, V., Fisher, L.C., Basso, D.M., and Popovich, P.G. (2004). Passive or active immunization with myelin basic protein impairs neurological function and exacerbates neuropathology after spinal cord injury in rats. J Neurosci 24, 3752-3761.

Jones, T.B., Basso, D.M., Sodhi, A., Pan, J.Z., Hart, R.P., MacCallum, R.C., Lee, S., Whitacre, C.C., and Popovich, P.G. (2002). Pathological CNS autoimmune disease triggered by traumatic spinal cord injury: implications for autoimmune vaccine therapy. J Neurosci 22, 2690-2700.

Kanneganti, T.D., Ozören, N., Body-Malapel, M., Amer, A., Park, J.H., Franchi, L., Whitfield, J., Barchet, W., Colonna, M., Vandenabeele, P., et al. (2006). Bacterial RNA and small antiviral compounds activate caspase-1 through cryopyrin/Nalp3. Nature 440, 233-236.

Kassmann, C.M., Lappe-Siefke, C., Baes, M., Brügger, B., Mildner, A., Werner, H.B., Natt, O., Michaelis, T., Prinz, M., Frahm, J., and Nave, K.A. (2007). Axonal loss and neuroinflammation caused by peroxisome-deficient oligodendrocytes. Nat Genet 39, 969-976.

Kataoka, A., Tozaki-Saitoh, H., Koga, Y., Tsuda, M., and Inoue, K. (2009). Activation of P2X7 receptors induces CCL3 production in microglial cells through transcription factor NFAT. J Neurochem 108, 115-125.

Kawai, T., Adachi, O., Ogawa, T., Takeda, K., and Akira, S. (1999). Unresponsiveness of MyD88-deficient mice to endotoxin. Immunity 11, 115-122.

Kawai, T., and Akira, S. (2005). Toll-like receptor downstream signaling. Arthritis Res Ther 7, 12-19.

Kawano, H., Kimura-Kuroda, J., Komuta, Y., Yoshioka, N., Li, H.P., Kawamura, K., Li, Y., and Raisman, G. (2012). Role of the lesion scar in the response to damage and repair of the central nervous system. Cell Tissue Res 349, 169-180.

Kawasaki, Y., Zhang, L., Cheng, J.K., and Ji, R.R. (2008). Cytokine mechanisms of central sensitization: distinct and overlapping role of interleukin-1beta, interleukin6 , and tumor necrosis factor-alpha in regulating synaptic and neuronal activity in the superficial spinal cord. J Neurosci 28, 5189-5194.

Kempuraj, D., Khan, M.M., Thangavel, R., Xiong, Z., Yang, E., and Zaheer, A. (2013). Glia maturation factor induces interleukin-33 release from astrocytes: implications for neurodegenerative diseases. J Neuroimmune Pharmacol 8, 643-650.

Kerschensteiner, M., Gallmeier, E., Behrens, L., Leal, V.V., Misgeld, T., Klinkert, W.E., Kolbeck, R., Hoppe, E., Oropeza-Wekerle, R.L., Bartke, I., et al. (1999). Activated human T cells, B cells, and monocytes produce brain-derived neurotrophic factor in 
vitro and in inflammatory brain lesions: a neuroprotective role of inflammation? J Exp Med 189, 865-870.

Khattri, R., Cox, T., Yasayko, S.A., and Ramsdell, F. (2003). An essential role for Scurfin in CD4+CD25+ T regulatory cells. Nat Immunol 4, 337-342.

Kierdorf, K., Erny, D., Goldmann, T., Sander, V., Schulz, C., Perdiguero, E.G., Wieghofer, P., Heinrich, A., Riemke, P., Hölscher, C., et al. (2013). Microglia emerge from erythromyeloid precursors via Pu.1- and Irf8-dependent pathways. Nat Neurosci 16, 273-280.

Kigerl, K.A., Gensel, J.C., Ankeny, D.P., Alexander, J.K., Donnelly, D.J., and Popovich, P.G. (2009). Identification of two distinct macrophage subsets with divergent effects causing either neurotoxicity or regeneration in the injured mouse spinal cord. J Neurosci 29, 13435-13444.

Kigerl, K.A., Lai, W., Rivest, S., Hart, R.P., Satoskar, A.R., and Popovich, P.G. (2007). Toll-like receptor (TLR)-2 and TLR-4 regulate inflammation, gliosis, and myelin sparing after spinal cord injury. J Neurochem 102, 37-50.

King, V.R., Phillips, J.B., Brown, R.A., and Priestley, J.V. (2004). The effects of treatment with antibodies to transforming growth factor beta1 and beta2 following spinal cord damage in the adult rat. Neuroscience 126, 173-183.

Kipnis, J., Avidan, H., Caspi, R.R., and Schwartz, M. (2004a). Dual effect of CD4(+)CD25(+) regulatory T cells in neurodegeneration: A dialogue with microglia. Proc Natl Acad Sci U S A 101, 14663-14669.

Kipnis, J., Avidan, H., Markovich, Y., Mizrahi, T., Hauben, E., Prigozhina, T.B., Slavin, S., and Schwartz, M. (2004b). Low-dose gamma-irradiation promotes survival of injured neurons in the central nervous system via homeostasis-driven proliferation of T cells. Eur J Neurosci 19, 1191-1198.

Kipnis, J., Cardon, M., Avidan, H., Lewitus, G.M., Mordechay, S., Rolls, A., Shani, Y., and Schwartz, M. (2004c). Dopamine, through the extracellular signal-regulated kinase pathway, downregulates CD4+CD25+ regulatory T-cell activity: implications for neurodegeneration. J Neurosci 24, 6133-6143.

Kipnis, J., Mizrahi, T., Hauben, E., Shaked, I., Shevach, E., and Schwartz, M. (2002a). Neuroprotective autoimmunity: Naturally occurring CD4(+)CD25(+) regulatory T cells suppress the ability to withstand injury to the central nervous system. Proceedings of the National Academy of Sciences of the United States of America 99, 15620-15625.

Kipnis, J., Mizrahi, T., Yoles, E., Ben-Nun, A., and Schwartz, M. (2002b). Myelin specific Th1 cells are necessary for post-traumatic protective autoimmunity. J Neuroimmunol 130, 78-85.

Kipnis, J., Yoles, E., Schori, H., Hauben, E., Shaked, I., and Schwartz, M. (2001). Neuronal survival after CNS insult is determined by a genetically encoded autoimmune response. Journal of Neuroscience 21, 4564-4571.

Kita, T., Liu, L., Tanaka, N., and Kinoshita, Y. (1997). The expression of tumor necrosis factor-alpha in the rat brain after fluid percussive injury. Int J Legal Med 110, 305-311. 
Kleinschnitz, C., Kraft, P., Dreykluft, A., Hagedorn, I., Gobel, K., Schuhmann, M.K., Langhauser, F., Helluy, X., Schwarz, T., Bittner, S., et al. (2013). Regulatory T cells are strong promoters of acute ischemic stroke in mice by inducing dysfunction of the cerebral microvasculature. Blood 121, 679-691.

Koeberle, P.D., Gauldie, J., and Ball, A.K. (2004). Effects of adenoviral-mediated gene transfer of interleukin-10, interleukin-4, and transforming growth factor-beta on the survival of axotomized retinal ganglion cells. Neuroscience 125, 903-920.

Komatsu, N., Mariotti-Ferrandiz, M.E., Wang, Y., Malissen, B., Waldmann, H., and Hori, S. (2009). Heterogeneity of natural Foxp3+ T cells: a committed regulatory Tcell lineage and an uncommitted minor population retaining plasticity. Proc Natl Acad Sci U S A 106, 1903-1908.

Kraft, A.D., McPherson, C.A., and Harry, G.J. (2009). Heterogeneity of microglia and TNF signaling as determinants for neuronal death or survival. Neurotoxicology 30, 785-793.

Kurimoto, T., Yin, Y., Habboub, G., Gilbert, H.Y., Li, Y., Nakao, S., Hafezi-Moghadam, A., and Benowitz, L.I. (2013). Neutrophils express oncomodulin and promote optic nerve regeneration. J Neurosci 33, 14816-14824.

Kühn, R., Löhler, J., Rennick, D., Rajewsky, K., and Müller, W. (1993). Interleukin-10deficient mice develop chronic enterocolitis. Cell 75, 263-274.

Lahl, K., Loddenkemper, C., Drouin, C., Freyer, J., Arnason, J., Eberl, G., Hamann, A., Wagner, H., Huehn, J., and Sparwasser, T. (2007). Selective depletion of Foxp3(+) regulatory $\mathrm{T}$ cells induces a scurfy-like disease. Journal of Experimental Medicine 204, 57-63.

Laird, M.D., Shields, J.S., Sukumari-Ramesh, S., Kimbler, D.E., Fessler, R.D., Shakir, B., Youssef, P., Yanasak, N., Vender, J.R., and Dhandapani, K.M. (2014). High mobility group box protein-1 promotes cerebral edema after traumatic brain injury via activation of toll-like receptor 4. Glia 62, 26-38.

Langlois, J.A., Rutland-Brown, W., and Thomas, K.E. (2005). The incidence of traumatic brain injury among children in the United States: differences by race. J Head Trauma Rehabil 20, 229-238.

Lappe-Siefke, C., Goebbels, S., Gravel, M., Nicksch, E., Lee, J., Braun, P.E., Griffiths, I.R., and Nave, K.A. (2003). Disruption of Cnp1 uncouples oligodendroglial functions in axonal support and myelination. Nat Genet 33, 366-374.

Lazarov-Spiegler, O., Solomon, A.S., Zeev-Brann, A.B., Hirschberg, D.L., Lavie, V., and Schwartz, M. (1996). Transplantation of activated macrophages overcomes central nervous system regrowth failure. FASEB J 10, 1296-1302.

Leavy, 0. (2013). Inflammasome: Turning on and off NLRP3. Nat Rev Immunol 13, 1. Lee, S.I., Jeong, S.R., Kang, Y.M., Han, D.H., Jin, B.K., Namgung, U., and Kim, B.G. (2010). Endogenous expression of interleukin-4 regulates macrophage activation and confines cavity formation after traumatic spinal cord injury. J Neurosci Res 88, 2409-2419.

Lehre, K.P., and Rusakov, D.A. (2002). Asymmetry of glia near central synapses favors presynaptically directed glutamate escape. Biophys J 83, 125-134. 
Leibovich, S.J., and Ross, R. (1975). The role of the macrophage in wound repair. A study with hydrocortisone and antimacrophage serum. Am J Pathol 78, 71-100.

Levi-Montalcini, R. (1987). The nerve growth factor 35 years later. Science 237, 1154-1162.

Liesz, A., Suri-Payer, E., Veltkamp, C., Doerr, H., Sommer, C., Rivest, S., Giese, T., and Veltkamp, R. (2009). Regulatory $T$ cells are key cerebroprotective immunomodulators in acute experimental stroke. Nat Med 15, 192-199.

Ling, C., Sandor, M., Suresh, M., and Fabry, Z. (2006). Traumatic injury and the presence of antigen differentially contribute to T-cell recruitment in the CNS. J Neurosci 26, 731-741.

Linker, R.A., Lee, D.H., Demir, S., Wiese, S., Kruse, N., Siglienti, I., Gerhardt, E., Neumann, H., Sendtner, M., Lühder, F., and Gold, R. (2010). Functional role of brainderived neurotrophic factor in neuroprotective autoimmunity: therapeutic implications in a model of multiple sclerosis. Brain 133, 2248-2263.

Lipton, S.A., and Nicotera, P. (1998). Calcium, free radicals and excitotoxins in neuronal apoptosis. Cell Calcium 23, 165-171.

Logan, A., Green, J., Hunter, A., Jackson, R., and Berry, M. (1999). Inhibition of glial scarring in the injured rat brain by a recombinant human monoclonal antibody to transforming growth factor-beta2. Eur J Neurosci 11, 2367-2374.

London, A., Cohen, M., and Schwartz, M. (2013). Microglia and monocyte-derived macrophages: functionally distinct populations that act in concert in CNS plasticity and repair. Front Cell Neurosci 7, 34.

Lozada, A., Maegele, M., Stark, H., Neugebauer, E.M., and Panula, P. (2005). Traumatic brain injury results in mast cell increase and changes in regulation of central histamine receptors. Neuropathol Appl Neurobiol 31, 150-162.

Lu, H.Z., Xu, L., Zou, J., Wang, Y.X., Ma, Z.W., Xu, X.M., and Lu, P.H. (2008). Effects of autoimmunity on recovery of function in adult rats following spinal cord injury. Brain Behavior and Immunity 22, 1217-1230.

Lööv, C., Hillered, L., Ebendal, T., and Erlandsson, A. (2012). Engulfing astrocytes protect neurons from contact-induced apoptosis following injury. PLoS One 7, e33090.

Ma, J., Huang, S., Qin, S., and You, C. (2012). Progesterone for acute traumatic brain injury. Cochrane Database Syst Rev 10, CD008409.

Madsen, L., Labrecque, N., Engberg, J., Dierich, A., Svejgaard, A., Benoist, C., Mathis, D., and Fugger, L. (1999). Mice lacking all conventional MHC class II genes. Proc Natl Acad Sci U S A 96, 10338-10343.

Mantovani, A., Cassatella, M.A., Costantini, C., and Jaillon, S. (2011). Neutrophils in the activation and regulation of innate and adaptive immunity. Nat Rev Immunol 11, 519-531.

Mariathasan, S., Newton, K., Monack, D.M., Vucic, D., French, D.M., Lee, W.P., RooseGirma, M., Erickson, S., and Dixit, V.M. (2004). Differential activation of the inflammasome by caspase-1 adaptors ASC and Ipaf. Nature 430, 213-218. 
Martinon, F., Pétrilli, V., Mayor, A., Tardivel, A., and Tschopp, J. (2006). Goutassociated uric acid crystals activate the NALP3 inflammasome. Nature 440, 237241.

Matzinger, P. (1994). Tolerance, danger, and the extended family. Annu Rev Immunol 12, 991-1045.

McKerracher, L., David, S., Jackson, D.L., Kottis, V., Dunn, R.J., and Braun, P.E. (1994). IDENTIFICATION OF MYELIN-ASSOCIATED GLYCOPROTEIN AS A MAJOR MYELINDERIVED INHIBITOR OF NEURITE GROWTH. Neuron 13, 805-811.

McPhail, L.T., Stirling, D.P., Tetzlaff, W., Kwiecien, J.M., and Ramer, M.S. (2004). The contribution of activated phagocytes and myelin degeneration to axonal retraction/dieback following spinal cord injury. Eur J Neurosci 20, 1984-1994.

Meythaler, J.M., Peduzzi, J.D., Eleftheriou, E., and Novack, T.A. (2001). Current concepts: diffuse axonal injury-associated traumatic brain injury. Arch Phys Med Rehabil 82, 1461-1471.

Mildner, A., Schmidt, H., Nitsche, M., Merkler, D., Hanisch, U.K., Mack, M., Heikenwalder, M., Brück, W., Priller, J., and Prinz, M. (2007). Microglia in the adult brain arise from Ly-6ChiCCR2+ monocytes only under defined host conditions. Nat Neurosci 10, 1544-1553.

Miller, S.D., McMahon, E.J., Schreiner, B., and Bailey, S.L. (2007). Antigen presentation in the CNS by myeloid dendritic cells drives progression of relapsing experimental autoimmune encephalomyelitis. Ann N Y Acad Sci 1103, 179-191.

Minkiewicz, J., de Rivero Vaccari, J.P., and Keane, R.W. (2013). Human astrocytes express a novel NLRP2 inflammasome. Glia 61, 1113-1121.

Miron, V.E., Boyd, A., Zhao, J.W., Yuen, T.J., Ruckh, J.M., Shadrach, J.L., van Wijngaarden, P., Wagers, A.J., Williams, A., Franklin, R.J., and Ffrench-Constant, C. (2013). M2 microglia and macrophages drive oligodendrocyte differentiation during CNS remyelination. Nat Neurosci.

Moalem, G., Leibowitz-Amit, R., Yoles, E., Mor, F., Cohen, I.R., and Schwartz, M. (1999). Autoimmune $T$ cells protect neurons from secondary degeneration after central nervous system axotomy. Nat Med 5, 49-55.

Mohrs, K., Wakil, A.E., Killeen, N., Locksley, R.M., and Mohrs, M. (2005). A Two-Step Process for Cytokine Production Revealed by IL-4 Dual-Reporter Mice. Immunity 23, 419-429.

Moll, C., Mourre, C., Lazdunski, M., and Ulrich, J. (1991). Increase of sodium channels in demyelinated lesions of multiple sclerosis. Brain Res 556, 311-316.

Moon, L.D., and Fawcett, J.W. (2001). Reduction in CNS scar formation without concomitant increase in axon regeneration following treatment of adult rat brain with a combination of antibodies to TGFbeta1 and beta2. Eur J Neurosci 14, 16671677.

Mortha, A., Chudnovskiy, A., Hashimoto, D., Bogunovic, M., Spencer, S.P., Belkaid, Y., and Merad, M. (2014). Microbiota-dependent crosstalk between macrophages and ILC3 promotes intestinal homeostasis. Science 343, 1249288. 
Mucida, D., Park, Y., Kim, G., Turovskaya, O., Scott, I., Kronenberg, M., and Cheroutre, H. (2007). Reciprocal TH17 and regulatory $\mathrm{T}$ cell differentiation mediated by retinoic acid. Science 317, 256-260.

Nagelhus, E.A., and Ottersen, O.P. (2013). Physiological roles of aquaporin-4 in brain. Physiol Rev 93, 1543-1562.

Neill, D.R., Wong, S.H., Bellosi, A., Flynn, R.J., Daly, M., Langford, T.K., Bucks, C., Kane, C.M., Fallon, P.G., Pannell, R., et al. (2010). Nuocytes represent a new innate effector leukocyte that mediates type-2 immunity. Nature 464, 1367-1370.

Nesić, D., and Vukmanović, S. (1998). MHC class I is required for peripheral accumulation of CD8+ thymic emigrants. J Immunol 160, 3705-3712.

Nguyen, H.X., Galvan, M.D., and Anderson, A.J. (2008). Characterization of early and terminal complement proteins associated with polymorphonuclear leukocytes in vitro and in vivo after spinal cord injury. J Neuroinflammation 5, 26.

Nikolaeva, M.A., Mukherjee, B., and Stys, P.K. (2005). Na+-dependent sources of intra-axonal $\mathrm{Ca} 2+$ release in rat optic nerve during in vitro chemical ischemia. J Neurosci 25, 9960-9967.

Oka, T., Hikoso, S., Yamaguchi, O., Taneike, M., Takeda, T., Tamai, T., Oyabu, J., Murakawa, T., Nakayama, H., Nishida, K., et al. (2012). Mitochondrial DNA that escapes from autophagy causes inflammation and heart failure. Nature 485, 251255.

Okada, S., Nakamura, M., Katoh, H., Miyao, T., Shimazaki, T., Ishii, K., Yamane, J., Yoshimura, A., Iwamoto, Y., Toyama, Y., and Okano, H. (2006). Conditional ablation of Stat3 or Socs3 discloses a dual role for reactive astrocytes after spinal cord injury. Nat Med 12, 829-834.

Okuma, Y., Liu, K., Wake, H., Liu, R., Nishimura, Y., Hui, Z., Teshigawara, K., Haruma, J., Yamamoto, Y., Yamamoto, H., et al. (2014). Glycyrrhizin inhibits traumatic brain injury by reducing HMGB1-RAGE interaction. Neuropharmacology 85, 18-26.

Okuma, Y., Liu, K., Wake, H., Zhang, J., Maruo, T., Date, I., Yoshino, T., Ohtsuka, A., Otani, N., Tomura, S., et al. (2012). Anti-high mobility group box-1 antibody therapy for traumatic brain injury. Ann Neurol 72, 373-384.

Oppenheim, J.J., Tewary, P., de la Rosa, G., and Yang, D. (2007). Alarmins initiate host defense. Adv Exp Med Biol 601, 185-194.

Oshima, T., Lee, S., Sato, A., Oda, S., Hirasawa, H., and Yamashita, T. (2009). TNFalpha contributes to axonal sprouting and functional recovery following traumatic brain injury. Brain Res 1290, 102-110.

Pan, Y., Chen, X.Y., Zhang, Q.Y., and Kong, L.D. (2014). Microglial NLRP3 inflammasome activation mediates IL-1 $\beta$-related inflammation in prefrontal cortex of depressive rats. Brain Behav Immun.

Panenka, W., Jijon, H., Herx, L.M., Armstrong, J.N., Feighan, D., Wei, T., Yong, V.W., Ransohoff, R.M., and MacVicar, B.A. (2001). P2X7-like receptor activation in astrocytes increases chemokine monocyte chemoattractant protein-1 expression via mitogen-activated protein kinase. J Neurosci 21, 7135-7142. 
Parri, H.R., Gould, T.M., and Crunelli, V. (2001). Spontaneous astrocytic Ca2+ oscillations in situ drive NMDAR-mediated neuronal excitation. Nat Neurosci 4, 803812.

Pasare, C., and Medzhitov, R. (2004). Toll-dependent control mechanisms of CD4 T cell activation. Immunity 21, 733-741.

Paterson, P.Y. (1960). Transfer of allergic encephalomyelitis in rats by means of lymph node cells. J Exp Med 111, 119-136.

Paul, W.E., and Zhu, J. (2010). How are T(H)2-type immune responses initiated and amplified? Nat Rev Immunol 10, 225-235.

Pawliński, R., Sołtys, Z., Setkowicz, Z., Kubala, E., and Janeczko, K. (2000). Effects of intracerebral administration of IL-1beta on reactive behaviors of astrocytes and macrophages in the injured brain of newborn rats. Folia Histochem Cytobiol 38, 151-156.

Pedrazzi, M., Patrone, M., Passalacqua, M., Ranzato, E., Colamassaro, D., Sparatore, B., Pontremoli, S., and Melloni, E. (2007). Selective proinflammatory activation of astrocytes by high-mobility group box 1 protein signaling. J Immunol 179, 85258532.

Pellerin, L., Pellegri, G., Bittar, P.G., Charnay, Y., Bouras, C., Martin, J.L., Stella, N., and Magistretti, P.J. (1998). Evidence supporting the existence of an activity-dependent astrocyte-neuron lactate shuttle. Dev Neurosci 20, 291-299.

Pinteaux, E., Trotter, P., and Simi, A. (2009). Cell-specific and concentrationdependent actions of interleukin-1 in acute brain inflammation. Cytokine 45, 1-7.

Plemel, J.R., Wee Yong, V., and Stirling, D.P. (2014). Immune modulatory therapies for spinal cord injury - Past, present and future. Exp Neurol 258C, 91-104.

Popovich, P.G., Guan, Z., Wei, P., Huitinga, I., van Rooijen, N., and Stokes, B.T. (1999). Depletion of hematogenous macrophages promotes partial hindlimb recovery and neuroanatomical repair after experimental spinal cord injury. Exp Neurol 158, 351365.

Popovich, P.G., Wei, P., and Stokes, B.T. (1997a). Cellular inflammatory response after spinal cord injury in Sprague-Dawley and Lewis rats. J Comp Neurol 377, 443464.

Popovich, P.G., Wei, P., and Stokes, B.T. (1997b). Cellular inflammatory response after spinal cord injury in Sprague-Dawley and Lewis rats. Journal of Comparative Neurology 377, 443-464.

Prang, P., Del Turco, D., and Kapfhammer, J.P. (2001). Regeneration of entorhinal fibers in mouse slice cultures is age dependent and can be stimulated by NT-4, GDNF, and modulators of G-proteins and protein kinase C. Exp Neurol 169, 135-147. Proescholdt, M.G., Chakravarty, S., Foster, J.A., Foti, S.B., Briley, E.M., and Herkenham, M. (2002). Intracerebroventricular but not intravenous interleukin-1beta induces widespread vascular-mediated leukocyte infiltration and immune signal mRNA expression followed by brain-wide glial activation. Neuroscience 112, 731-749.

Ransohoff, R.M., and Brown, M.A. (2012). Innate immunity in the central nervous system. J Clin Invest 122, 1164-1171. 
Rapalino, O., Lazarov-Spiegler, O., Agranov, E., Velan, G.J., Yoles, E., Fraidakis, M., Solomon, A., Gepstein, R., Katz, A., Belkin, M., et al. (1998). Implantation of stimulated homologous macrophages results in partial recovery of paraplegic rats. Nat Med 4, 814-821.

Reichardt, L.F. (2006). Neurotrophin-regulated signalling pathways. Philos Trans R Soc Lond B Biol Sci 361, 1545-1564.

Ren, X., Akiyoshi, K., Vandenbark, A.A., Hurn, P.D., and Offner, H. (2010). CD4(+)FoxP3(+) regulatory T-cells in cerebral ischemic stroke. Metab Brain Dis.

Reynolds, A.D., Banerjee, R., Liu, J.N., Gendelman, H.E., and Mosley, R.L. (2007). Neuroprotective activities of CD4+CD25+ regulatory T cells in an animal model of Parkinson's disease. Journal of Leukocyte Biology 82, 1083-1094.

Rhodes, K.E., Raivich, G., and Fawcett, J.W. (2006). The injury response of oligodendrocyte precursor cells is induced by platelets, macrophages and inflammation-associated cytokines. Neuroscience 140, 87-100.

Ribeiro, M.e.C., Hirt, L., Bogousslavsky, J., Regli, L., and Badaut, J. (2006). Time course of aquaporin expression after transient focal cerebral ischemia in mice. J Neurosci Res 83, 1231-1240.

Rich, K.M. (1992). Neuronal death after trophic factor deprivation. J Neurotrauma 9 Suppl 1, S61-69.

Rigor, R.R., Beard, R.S., Litovka, O.P., and Yuan, S.Y. (2012). Interleukin-1ß-induced barrier dysfunction is signaled through PKC- $\theta$ in human brain microvascular endothelium. Am J Physiol Cell Physiol 302, C1513-1522.

Rivest, S. (2009). Regulation of innate immune responses in the brain. Nat Rev Immunol 9, 429-439.

Rubtsov, Y.P., Niec, R.E., Josefowicz, S., Li, L., Darce, J., Mathis, D., Benoist, C., and Rudensky, A.Y. (2010). Stability of the regulatory T cell lineage in vivo. Science 329, 1667-1671.

Ruffolo, C.F., Friedland, J.F., Dawson, D.R., Colantonio, A., and Lindsay, P.H. (1999). Mild traumatic brain injury from motor vehicle accidents: factors associated with return to work. Arch Phys Med Rehabil 80, 392-398.

Rutland-Brown, W., Langlois, J.A., Thomas, K.E., and Xi, Y.L. (2006). Incidence of traumatic brain injury in the United States, 2003. J Head Trauma Rehabil 21, 544548.

Sakaguchi, S., Sakaguchi, N., Asano, M., Itoh, M., and Toda, M. (1995). Immunologic self-tolerance maintained by activated $\mathrm{T}$ cells expressing IL-2 receptor alpha-chains (CD25). Breakdown of a single mechanism of self-tolerance causes various autoimmune diseases. J Immunol 155, 1151-1164.

Salter, M.W., and Beggs, S. (2014). Sublime Microglia: Expanding Roles for the Guardians of the CNS. Cell 158, 15-24.

Sansing, L.H., Harris, T.H., Kasner, S.E., Hunter, C.A., and Kariko, K. (2011). Neutrophil depletion diminishes monocyte infiltration and improves functional outcome after experimental intracerebral hemorrhage. Acta Neurochir Suppl 111, 173-178. 
Sato, A., Ohtaki, H., Tsumuraya, T., Song, D., Ohara, K., Asano, M., Iwakura, Y., Atsumi, T., and Shioda, S. (2012). Interleukin-1 participates in the classical and alternative activation of microglia/macrophages after spinal cord injury. J Neuroinflammation 9,65 .

Schafer, D.P., Lehrman, E.K., Kautzman, A.G., Koyama, R., Mardinly, A.R., Yamasaki, R., Ransohoff, R.M., Greenberg, M.E., Barres, B.A., and Stevens, B. (2012). Microglia sculpt postnatal neural circuits in an activity and complement-dependent manner. Neuron 74, 691-705.

Scherbel, U., Raghupathi, R., Nakamura, M., Saatman, K.E., Trojanowski, J.Q., Neugebauer, E., Marino, M.W., and McIntosh, T.K. (1999). Differential acute and chronic responses of tumor necrosis factor-deficient mice to experimental brain injury. Proc Natl Acad Sci U S A 96, 8721-8726.

Schmitz, J., Owyang, A., Oldham, E., Song, Y., Murphy, E., McClanahan, T.K., Zurawski, G., Moshrefi, M., Qin, J., Li, X., et al. (2005). IL-33, an interleukin-1-like cytokine that signals via the IL-1 receptor-related protein ST2 and induces T helper type 2associated cytokines. Immunity 23, 479-490.

Schmitz, N., Kurrer, M., and Kopf, M. (2003). The IL-1 receptor 1 is critical for Th2 cell type airway immune responses in a mild but not in a more severe asthma model. Eur J Immunol 33, 991-1000.

Schori, H., Lantner, F., Shachar, I., and Schwartz, M. (2002). Severe immunodeficiency has opposite effects on neuronal survival in glutamatesusceptible and -resistant mice: adverse effect of B cells. J Immunol 169, 2861-2865. Schori, H., Shechter, R., Shachar, I., and Schwartz, M. (2007). Genetic manipulation of CD74 in mouse strains of different backgrounds can result in opposite responses to central nervous system injury. J Immunol 178, 163-171.

Schulz, C., Gomez Perdiguero, E., Chorro, L., Szabo-Rogers, H., Cagnard, N., Kierdorf, K., Prinz, M., Wu, B., Jacobsen, S.E., Pollard, J.W., et al. (2012). A lineage of myeloid cells independent of Myb and hematopoietic stem cells. Science 336, 86-90.

Schwartz, M., and Kipnis, J. (2002). Autoimmunity on alert: naturally occurring regulatory CD4(+)CD25(+) T cells as part of the evolutionary compromise between a 'need' and a 'risk'. Trends Immunol 23, 530-534.

Scripter, J.L., Ko, J., Kow, K., Arimura, A., and Ide, C.F. (1997). Regulation by interleukin-1beta of formation of a line of delimiting astrocytes following prenatal trauma to the brain of the mouse. Exp Neurol 145, 329-341.

Seifert, G., Schilling, K., and Steinhäuser, C. (2006). Astrocyte dysfunction in neurological disorders: a molecular perspective. Nat Rev Neurosci 7, 194-206.

Sekhon, L.H.S., and Fehlings, M.G. (2001). Epidemiology, demographics, and pathophysiology of acute spinal cord injury. Spine 26, S2-S12.

Selmaj, K.W., and Raine, C.S. (1988). Tumor necrosis factor mediates myelin and oligodendrocyte damage in vitro. Ann Neurol 23, 339-346.

Semple, B.D., Bye, N., Rancan, M., Ziebell, J.M., and Morganti-Kossmann, M.C. (2010a). Role of CCL2 (MCP-1) in traumatic brain injury (TBI): evidence from severe TBI patients and CCL2-/- mice. J Cereb Blood Flow Metab 30, 769-782. 
Semple, B.D., Bye, N., Ziebell, J.M., and Morganti-Kossmann, M.C. (2010b). Deficiency of the chemokine receptor CXCR2 attenuates neutrophil infiltration and cortical damage following closed head injury. Neurobiol Dis 40, 394-403.

Serpe, C.J., Coers, S., Sanders, V.M., and Jones, K.J. (2003). CD4+ T, but not CD8+ or B, lymphocytes mediate facial motoneuron survival after facial nerve transection. Brain Behav Immun 17, 393-402.

Serpe, C.J., Kohm, A.P., Huppenbauer, C.B., Sanders, V.M., and Jones, K.J. (1999). Exacerbation of facial motoneuron loss after facial nerve transection in severe combined immunodeficient (scid) mice. J Neurosci 19, Rc7.

Setiady, Y.Y., Coccia, J.A., and Park, P.U. (2010). In vivo depletion of CD4+FOXP3+ Treg cells by the PC61 anti-CD25 monoclonal antibody is mediated by FcgammaRIII+ phagocytes. Eur J Immunol 40, 780-786.

Sewell, D.L., Nacewicz, B., Liu, F., Macvilay, S., Erdei, A., Lambris, J.D., Sandor, M., and Fabry, Z. (2004). Complement C3 and C5 play critical roles in traumatic brain cryoinjury: blocking effects on neutrophil extravasation by C5a receptor antagonist. J Neuroimmunol 155, 55-63.

Sharp, J., Frame, J., Siegenthaler, M., Nistor, G., and Keirstead, H.S. (2010). Human embryonic stem cell-derived oligodendrocyte progenitor cell transplants improve recovery after cervical spinal cord injury. Stem Cells 28, 152-163.

Shechter, R., London, A., Varol, C., Raposo, C., Cusimano, M., Yovel, G., Rolls, A., Mack, M., Pluchino, S., Martino, G., et al. (2009). Infiltrating blood-derived macrophages are vital cells playing an anti-inflammatory role in recovery from spinal cord injury in mice. PLoS Med 6, e1000113.

Shechter, R., Miller, O., Yovel, G., Rosenzweig, N., London, A., Ruckh, J., Kim, K.W., Klein, E., Kalchenko, V., Bendel, P., et al. (2013). Recruitment of beneficial M2 macrophages to injured spinal cord is orchestrated by remote brain choroid plexus. Immunity 38, 555-569.

Sherman, D.L., and Brophy, P.J. (2005). Mechanisms of axon ensheathment and myelin growth. Nat Rev Neurosci 6, 683-690.

Shi, Y., Evans, J.E., and Rock, K.L. (2003). Molecular identification of a danger signal that alerts the immune system to dying cells. Nature 425, 516-521.

Shimada, K., Crother, T.R., Karlin, J., Dagvadorj, J., Chiba, N., Chen, S., Ramanujan, V.K., Wolf, A.J., Vergnes, L., Ojcius, D.M., et al. (2012). Oxidized mitochondrial DNA activates the NLRP3 inflammasome during apoptosis. Immunity 36, 401-414.

Shornick, L.P., De Togni, P., Mariathasan, S., Goellner, J., Strauss-Schoenberger, J., Karr, R.W., Ferguson, T.A., and Chaplin, D.D. (1996). Mice deficient in IL-1beta manifest impaired contact hypersensitivity to trinitrochlorobenzone. J Exp Med 183, $1427-1436$.

Slaets, H., Nelissen, S., Janssens, K., Vidal, P.M., Lemmens, E., Stinissen, P., Hendrix, S., and Hellings, N. (2014). Oncostatin M Reduces Lesion Size and Promotes Functional Recovery and Neurite Outgrowth After Spinal Cord Injury. Mol Neurobiol.

Smaili, S., Hirata, H., Ureshino, R., Monteforte, P.T., Morales, A.P., Muler, M.L., Terashima, J., Oseki, K., Rosenstock, T.R., Lopes, G.S., and Bincoletto, C. (2009). 
Calcium and cell death signaling in neurodegeneration and aging. An Acad Bras Cienc 81, 467-475.

Spira, M.E., Benbassat, D., and Dormann, A. (1993). Resealing of the proximal and distal cut ends of transected axons: electrophysiological and ultrastructural analysis. J Neurobiol 24, 300-316.

Spranger, M., Lindholm, D., Bandtlow, C., Heumann, R., Gnahn, H., Näher-Noé, M., and Thoenen, H. (1990). Regulation of Nerve Growth Factor (NGF) Synthesis in the Rat Central Nervous System: Comparison between the Effects of Interleukin-1 and Various Growth Factors in Astrocyte Cultures and in vivo. Eur J Neurosci 2, 69-76.

Sticozzi, C., Belmonte, G., Meini, A., Carbotti, P., Grasso, G., and Palmi, M. (2013). IL$1 \beta$ induces GFAP expression in vitro and in vivo and protects neurons from traumatic injury-associated apoptosis in rat brain striatum via $\mathrm{NF \kappa B} / \mathrm{Ca}^{2+}$ calmodulin/ERK mitogen-activated protein kinase signaling pathway. Neuroscience 252, 367-383.

Stirling, D.P., Liu, S., Kubes, P., and Yong, V.W. (2009). Depletion of Ly6G/Gr-1 leukocytes after spinal cord injury in mice alters wound healing and worsens neurological outcome. J Neurosci 29, 753-764.

Strle, K., Zhou, J.H., Shen, W.H., Broussard, S.R., Johnson, R.W., Freund, G.G., Dantzer, R., and Kelley, K.W. (2001). Interleukin-10 in the brain. Crit Rev Immunol 21, 427449.

Sun, X.J., Wang, L.M., Zhang, Y., Yenush, L., Myers, M.G., Glasheen, E., Lane, W.S., Pierce, J.H., and White, M.F. (1995). Role of IRS-2 in insulin and cytokine signalling. Nature 377, 173-177.

Sun, Y., Xu, C.C., Li, J., Guan, X.Y., Gao, L., Ma, L.X., Li, R.X., Peng, Y.W., and Zhu, G.P. (2013). Transplantation of oligodendrocyte precursor cells improves locomotion deficits in rats with spinal cord irradiation injury. PLoS One 8, e57534.

Suresh, M.V., Yu, B., Machado-Aranda, D., Bender, M.D., Ochoa-Frongia, L., Helinski, J.D., Davidson, B.A., Knight, P.R., Hogaboam, C.M., Moore, B.B., and Raghavendran, K. (2012). Role of macrophage chemoattractant protein-1 in acute inflammation after lung contusion. Am J Respir Cell Mol Biol 46, 797-806.

Sydenham, E., Roberts, I., and Alderson, P. (2009). Hypothermia for traumatic head injury. Cochrane Database Syst Rev, CD001048.

Takeda, K., Tanaka, T., Shi, W., Matsumoto, M., Minami, M., Kashiwamura, S., Nakanishi, K., Yoshida, N., Kishimoto, T., and Akira, S. (1996a). Essential role of Stat6 in IL-4 signalling. Nature 380, 627-630.

Takeda, S., Rodewald, H.R., Arakawa, H., Bluethmann, H., and Shimizu, T. (1996b). MHC class II molecules are not required for survival of newly generated CD4+ T cells, but affect their long-term life span. Immunity 5, 217-228.

Teng, Y.D., Choi, H., Onario, R.C., Zhu, S., Desilets, F.C., Lan, S., Woodard, E.J., Snyder, E.Y., Eichler, M.E., and Friedlander, R.M. (2004). Minocycline inhibits contusiontriggered mitochondrial cytochrome c release and mitigates functional deficits after spinal cord injury. Proc Natl Acad Sci U S A 101, 3071-3076. 
Tenorio, E.P., Fernandez, J., Olguin, J.E., and Saavedra, R. (2011). Depletion with PC61 mAb before Toxoplasma gondii infection eliminates mainly Tregs in BALB/c mice but activated cells in C57BL/6J mice. FEMS Immunol Med Microbiol.

Thompson, C.D., Zurko, J.C., Hanna, B.F., Hellenbrand, D.J., and Hanna, A. (2013). The therapeutic role of interleukin-10 after spinal cord injury. J Neurotrauma 30, 13111324.

Thornton, P., Pinteaux, E., Allan, S.M., and Rothwell, N.J. (2008). Matrix metalloproteinase-9 and urokinase plasminogen activator mediate interleukin-1induced neurotoxicity. Mol Cell Neurosci 37, 135-142.

Tian, L., Altin, J.A., Makaroff, L.E., Franckaert, D., Cook, M.C., Goodnow, C.C., Dooley, J., and Liston, A. (2011). Foxp $3^{+}$regulatory $T$ cells exert asymmetric control over murine helper responses by inducing Th2 cell apoptosis. Blood 118, 1845-1853.

Tikhonova, A.N., Van Laethem, F., Hanada, K., Lu, J., Pobezinsky, L.A., Hong, C., Guinter, T.I., Jeurling, S.K., Bernhardt, G., Park, J.H., et al. (2012). $\alpha \beta$ T cell receptors that do not undergo major histocompatibility complex-specific thymic selection possess antibody-like recognition specificities. Immunity 36, 79-91.

Tikka, T., Fiebich, B.L., Goldsteins, G., Keinanen, R., and Koistinaho, J. (2001). Minocycline, a tetracycline derivative, is neuroprotective against excitotoxicity by inhibiting activation and proliferation of microglia. J Neurosci 21, 2580-2588.

Tjoa, T., Strausbaugh, H.J., Maida, N., Dazin, P.F., Rosen, S.D., and Noble-Haeusslein, L.J. (2003). The use of flow cytometry to assess neutrophil infiltration in the injured murine spinal cord. J Neurosci Methods 129, 49-59.

Tomura, S., de Rivero Vaccari, J.P., Keane, R.W., Bramlett, H.M., and Dietrich, W.D. (2012). Effects of therapeutic hypothermia on inflammasome signaling after traumatic brain injury. J Cereb Blood Flow Metab 32, 1939-1947.

Tyzack, G.E., Sitnikov, S., Barson, D., Adams-Carr, K.L., Lau, N.K., Kwok, J.C., Zhao, C., Franklin, R.J., Karadottir, R.T., Fawcett, J.W., and Lakatos, A. (2014). Astrocyte response to motor neuron injury promotes structural synaptic plasticity via STAT3regulated TSP-1 expression. Nat Commun 5, 4294.

Uçeyler, N., Tscharke, A., and Sommer, C. (2008). Early cytokine gene expression in mouse CNS after peripheral nerve lesion. Neurosci Lett 436, 259-264.

Van Dyken, S.J., and Locksley, R.M. (2013). Interleukin-4- and interleukin-13mediated alternatively activated macrophages: roles in homeostasis and disease. Annu Rev Immunol 31, 317-343.

van Landeghem, F.K., Weiss, T., Oehmichen, M., and von Deimling, A. (2006). Decreased expression of glutamate transporters in astrocytes after human traumatic brain injury. J Neurotrauma 23, 1518-1528.

Vicario-Abejón, C., Owens, D., McKay, R., and Segal, M. (2002). Role of neurotrophins in central synapse formation and stabilization. Nat Rev Neurosci 3, 965-974.

Viviani, B., Bartesaghi, S., Gardoni, F., Vezzani, A., Behrens, M.M., Bartfai, T., Binaglia, M., Corsini, E., Di Luca, M., Galli, C.L., and Marinovich, M. (2003). Interleukin-1beta enhances NMDA receptor-mediated intracellular calcium increase through activation of the Src family of kinases. J Neurosci 23, 8692-8700. 
Walsh, J.G., Muruve, D.A., and Power, C. (2014). Inflammasomes in the CNS. Nat Rev Neurosci 15, 84-97.

Walsh, J.T., and Kipnis, J. (2011). Regulatory T cells in CNS injury: the simple, the complex and the confused. Trends Mol Med.

Wang, K.C., Kim, J.A., Sivasankaran, R., Segal, R., and He, Z. (2002). P75 interacts with the Nogo receptor as a co-receptor for Nogo, MAG and OMgp. Nature 420, 74-78.

Warren, G.L., O'Farrell, L., Summan, M., Hulderman, T., Mishra, D., Luster, M.I., Kuziel, W.A., and Simeonova, P.P. (2004). Role of CC chemokines in skeletal muscle functional restoration after injury. Am J Physiol Cell Physiol 286, C1031-1036.

Watkins, T.A., and Barres, B.A. (2002). Nerve regeneration: regrowth stumped by shared receptor. Curr Biol 12, R654-656.

Wheeler, K., Tardif, S., Rival, C., Luu, B., Bui, E., Del Rio, R., Teuscher, C., Sparwasser, T., Hardy, D., and Tung, K.S. (2011). Regulatory T cells control tolerogenic versus autoimmune response to sperm in vasectomy. Proc Natl Acad Sci U S A 108, 75117516.

Willis, C.L., Leach, L., Clarke, G.J., Nolan, C.C., and Ray, D.E. (2004). Reversible disruption of tight junction complexes in the rat blood-brain barrier, following transitory focal astrocyte loss. Glia 48, 1-13.

Woodhams, P.L., and Atkinson, D.J. (1996). Regeneration of entorhino-dentate projections in organotypic slice cultures: mode of axonal regrowth and effects of growth factors. Exp Neurol 140, 68-78.

Wu, B., Sun, L., Li, P., Tian, M., Luo, Y., and Ren, X. (2012). Transplantation of oligodendrocyte precursor cells improves myelination and promotes functional recovery after spinal cord injury. Injury 43, 794-801.

Xia, M., and Zhu, Y. (2013). FOXO3a involvement in the release of TNF- $\alpha$ stimulated by ATP in spinal cord astrocytes. J Mol Neurosci 51, 792-804.

Xin, J., Wainwright, D.A., Mesnard, N.A., Serpe, C.J., Sanders, V.M., and Jones, K.J. (2011). IL-10 within the CNS is necessary for CD4+ $T$ cells to mediate neuroprotection. Brain Behav Immun 25, 820-829.

Xiong, X., Barreto, G.E., Xu, L., Ouyang, Y.B., Xie, X., and Giffard, R.G. (2011). Increased brain injury and worsened neurological outcome in interleukin-4 knockout mice after transient focal cerebral ischemia. Stroke 42, 2026-2032.

Yasuoka, S., Kawanokuchi, J., Parajuli, B., Jin, S., Doi, Y., Noda, M., Sonobe, Y., Takeuchi, H., Mizuno, T., and Suzumura, A. (2011). Production and functions of IL-33 in the central nervous system. Brain Res 1385, 8-17.

Yoles, E., Hauben, E., Palgi, O., Agranov, E., Gothilf, A., Cohen, A., Kuchroo, V., Cohen, I.R., Weiner, H., and Schwartz, M. (2001). Protective autoimmunity is a physiological response to CNS trauma. J Neurosci 21, 3740-3748.

Yoles, E., and Schwartz, M. (1998). Degeneration of spared axons following partial white matter lesion: implications for optic nerve neuropathies. Experimental Neuroloy 153, 1-7.

Yong, V.W., Wells, J., Giuliani, F., Casha, S., Power, C., and Metz, L.M. (2004). The promise of minocycline in neurology. Lancet Neurol 3, 744-751. 
Zamanian, J.L., Xu, L., Foo, L.C., Nouri, N., Zhou, L., Giffard, R.G., and Barres, B.A. (2012). Genomic analysis of reactive astrogliosis. J Neurosci 32, 6391-6410.

Zhai, C.L., Zhang, M.Q., Zhang, Y., Xu, H.X., Wang, J.M., An, G.P., Wang, Y.Y., and Li, L. (2012). Glycyrrhizin protects rat heart against ischemia-reperfusion injury through blockade of HMGB1-dependent phospho-JNK/Bax pathway. Acta Pharmacol Sin 33, 1477-1487.

Zhang, Q., Raoof, M., Chen, Y., Sumi, Y., Sursal, T., Junger, W., Brohi, K., Itagaki, K., and Hauser, C.J. (2010). Circulating mitochondrial DAMPs cause inflammatory responses to injury. Nature 464, 104-107.

Zhao, W., Beers, D.R., Liao, B., Henkel, J.S., and Appel, S.H. (2012). Regulatory T lymphocytes from ALS mice suppress microglia and effector T lymphocytes through different cytokine-mediated mechanisms. Neurobiol Dis 48, 418-428.

Zhao, W., Xie, W., Xiao, Q., Beers, D.R., and Appel, S.H. (2006). Protective effects of an anti-inflammatory cytokine, interleukin-4, on motoneuron toxicity induced by activated microglia. J Neurochem 99, 1176-1187.

Zheng, W., and Flavell, R.A. (1997). The transcription factor GATA-3 is necessary and sufficient for Th2 cytokine gene expression in CD4 T cells. Cell 89, 587-596.

Zhou, X., Bailey-Bucktrout, S.L., Jeker, L.T., Penaranda, C., Martinez-Llordella, M., Ashby, M., Nakayama, M., Rosenthal, W., and Bluestone, J.A. (2009a). Instability of the transcription factor Foxp3 leads to the generation of pathogenic memory $\mathrm{T}$ cells in vivo. Nat Immunol 10, 1000-1007.

Zhou, X., Kong, N., Wang, J., Fan, H., Zou, H., Horwitz, D., Brand, D., Liu, Z., and Zheng, S.G. (2010). Cutting edge: all-trans retinoic acid sustains the stability and function of natural regulatory $\mathrm{T}$ cells in an inflammatory milieu. In J Immunol (United States), pp. 2675-2679.

Zhou, Z., Peng, X., Insolera, R., Fink, D.J., and Mata, M. (2009b). IL-10 promotes neuronal survival following spinal cord injury. Exp Neurol 220, 183-190.

Ziegenfuss, J.S., Doherty, J., and Freeman, M.R. (2012). Distinct molecular pathways mediate glial activation and engulfment of axonal debris after axotomy. Nat Neurosci 15, 979-987.

Zuo, J., Neubauer, D., Graham, J., Krekoski, C.A., Ferguson, T.A., and Muir, D. (2002). Regeneration of axons after nerve transection repair is enhanced by degradation of chondroitin sulfate proteoglycan. Exp Neurol 176, 221-228.

Zweifel, L.S., Kuruvilla, R., and Ginty, D.D. (2005). Functions and mechanisms of retrograde neurotrophin signalling. Nat Rev Neurosci 6, 615-625. 


\section{Appendix II: Supplemental Figures and Legends}
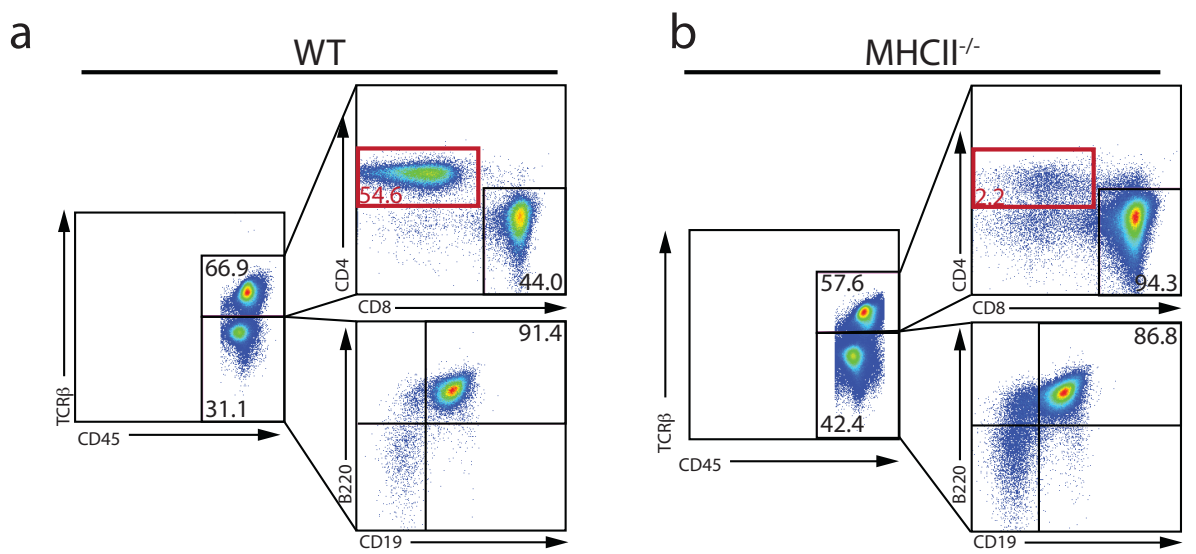

Supplementary Figure 1: $M H C-I \Gamma^{-}$mice have normal $B$ cell and $\mathrm{CD}^{+} T$ cell populations, but lack $\mathrm{CD}^{+} \mathrm{T}$ cells. Flow cytometry of the deep cervical lymph nodes of wild type (a) and $\mathrm{MHCII}^{-/-}$(b) mice showing $\mathrm{CD}^{+}$and $\mathrm{CD}^{+}$lymphocytes in the $\mathrm{TCR} \beta^{+}$ population and $\mathrm{CD} 19^{+} \mathrm{B} 220^{+} \mathrm{B}$ cells in the $\mathrm{CD} 45^{+} \mathrm{TCR} \beta^{-}$population. Numbers indicate percent $\mathrm{CD}^{+}$(upper left) and $\mathrm{CD}^{+}$(lower right), as a percentage of $\mathrm{TCR} \beta^{+}$cells and $\mathrm{CD} 19^{+} \mathrm{B} 220^{+} \mathrm{B}$ cells as a percentage of the $\mathrm{CD} 45^{+} \mathrm{TCR} \beta^{-}$population. 
a

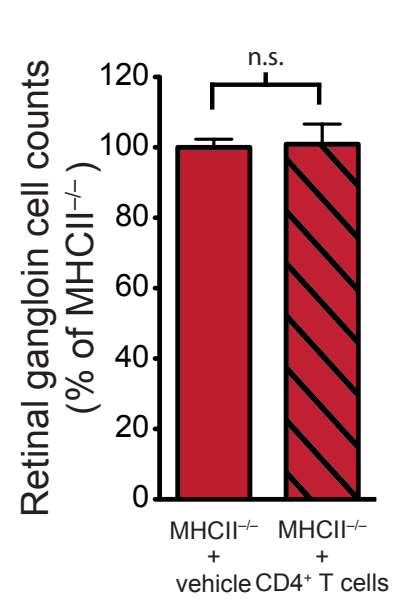

d

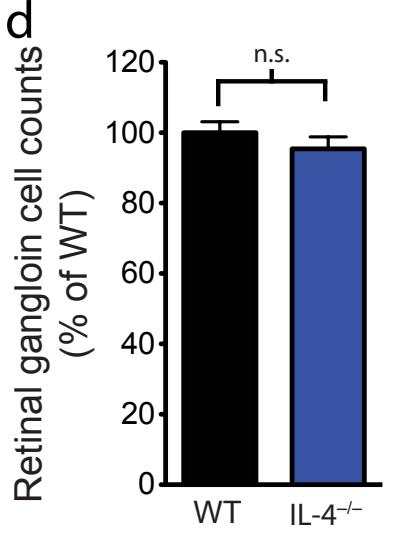

b

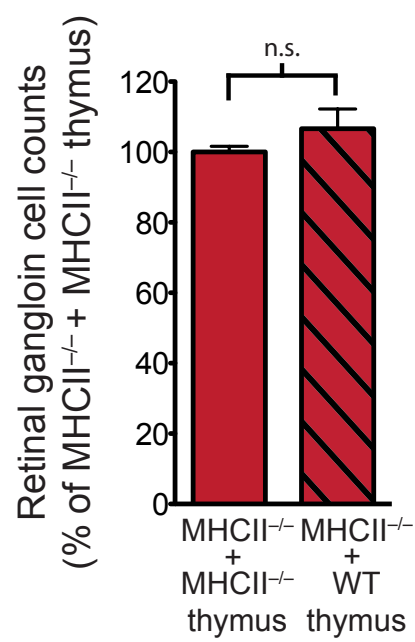

e

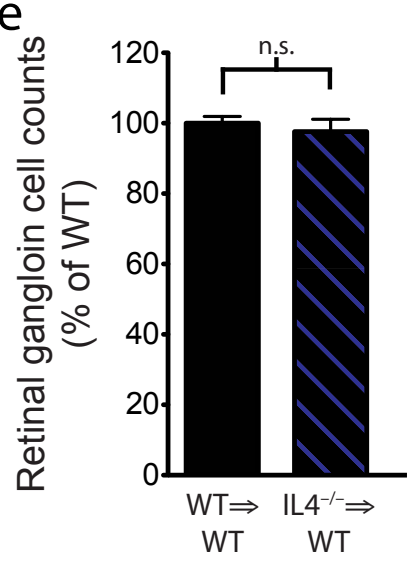

C

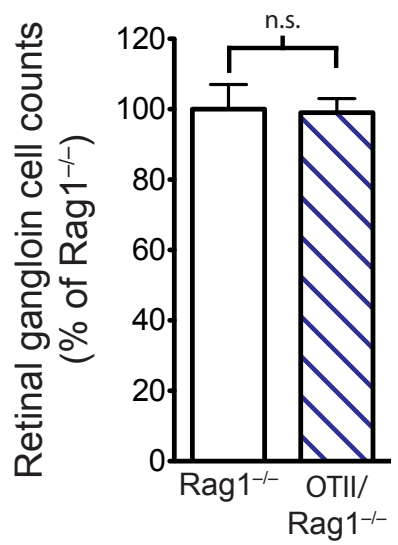

f

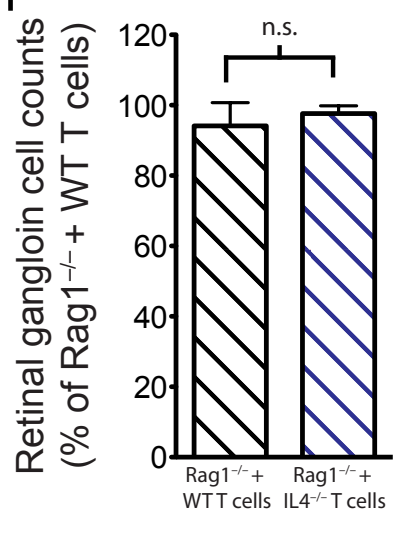

g

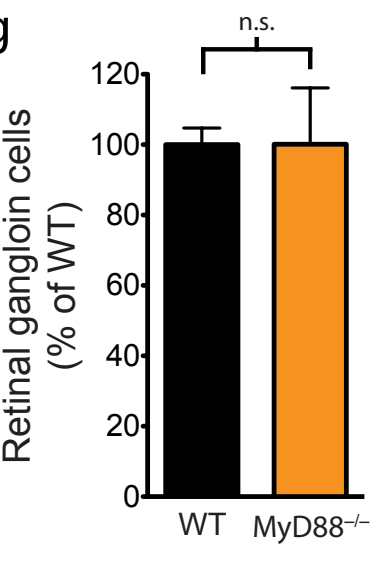

$\mathrm{h}$

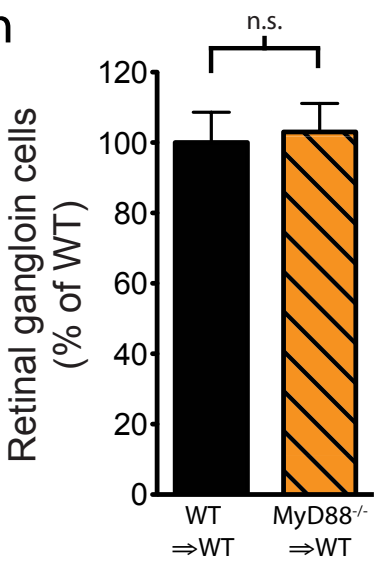

Supplementary Figure 2: No difference in contralateral, uninjured retinas of all mouse strains and experimental manipulations examined in this manuscript. (a) $\mathrm{MHCII}^{-/-}$mice 
injected with $3 \times 10^{6}$ wild type $\mathrm{CD} 4^{+} \mathrm{T}$ cells on the day of injury exhibit no difference in neuronal counts in the uninjured retinas compared to those injected with saline. Retinal ganglion cell counts $\left(\%\right.$ of $\left.\mathrm{MHCII}^{--}\right)$of $\mathrm{MHCII}^{-/-}$mice and $\mathrm{MHCII}^{-/-}$mice injected with wild type $\mathrm{CD}^{+} \mathrm{T}$ cells as assessed by Fluoro-Gold staining ( $\mathrm{n}=3$, wild type thymus; $\mathrm{n}=$ $3 \mathrm{MHCII}^{-/-}$thymus; Student's t-test). (b) $\mathrm{MHCII}^{-/-}$mice implanted with wild type thymi exhibit no difference in neuronal counts in the uninjured retinas compared to those implanted with $\mathrm{MHCII}^{-/-}$thymi. Retinal ganglion cell counts $\left(\%\right.$ of $\mathrm{MHCII}^{-/-}+\mathrm{MHCII}^{-/-}$ thymus) of $\mathrm{MHCII}^{--}$mice implanted with either a $\mathrm{MHCII}^{-/-}$or wild type thymi (six weeks after implantation) as assessed by Fluoro-Gold staining ( $\mathrm{n}=5$, wild type thymus; $\mathrm{n}$ $=8 \mathrm{MHCII}^{-/-}$thymus; Student's t-test). (c) OTII/Rag $1^{-/-}$mice exhibit no difference in retinal ganglion cell number in the uninjured retinas compared to Rag $1^{-/-}$mice. Retinal ganglion cell counts ( $\%$ of $\operatorname{Rag} 1^{--}$) of $\operatorname{Rag} 1^{-/-}$and OTII/Rag1 $1^{-/-}$mice assessed by FluoroGold staining ( $\mathrm{n}=3, \operatorname{Rag} 1^{--} ; \mathrm{n}=3$, OTII $/ \operatorname{Rag} 1^{--}$; Student's t-test). (d) $\mathrm{IL}_{-} 4^{-/-}$mice exhibit no difference in retinal ganglion cell number in the contralateral retinas compared to wild type mice. Bar graphs represent retinal ganglion cell counts ( $\%$ of wild type) of IL- $4^{-/-}$or wild type mice, assessed by Fluoro-Gold staining ( $\mathrm{n}=5$, wild type; $\mathrm{n}=5, \mathrm{IL}-4^{-}$ ${ }^{\prime-}$; Student's t-test). (e) Mice transplanted with IL- $4^{-/-}$bone marrow exhibit no difference in retinal ganglion cell number in the contralateral retinas compared to those transplanted with wild type bone marrow. Retinal ganglion cell counts (\% of wild type $\Rightarrow$ wild type) of wild type $\Rightarrow$ wild type or IL- $4^{--} \Rightarrow$ wild type bone marrow chimeras. Bone marrow was allowed to engraft for 6 weeks before optic nerve injury, and retinal ganglion cell counts were assessed by Fluoro-Gold staining ( $\mathrm{n}=3$, wild type bone marrow; $\mathrm{n}=3$, IL$4^{-/-}$bone marrow; Student's t-test). (f) $\mathrm{Rag} 1^{-/-}$mice receiving IL- $4^{-/} \mathrm{CD} 4^{+} \mathrm{T}$ cells 
demonstrate no difference in retinal ganglion cell number in the contralateral retinas compared to $\mathrm{Rag} 1^{-/-}$mice receiving wild type $\mathrm{T}$ cells. Retinal ganglion cell counts $(\%$ of $\operatorname{Rag}^{-/-}$mice injected with wild type $\mathrm{T}$ cells) of $\operatorname{Rag} 1^{-/-}$mice injected with either wild type or IL-4 $4^{-/-} \mathrm{CD} 4^{+} \mathrm{T}$ cells 3 weeks before optic nerve injury. Retinal ganglion cell counts were assessed by Fluoro-Gold staining ( $\mathrm{n}=7$, wild type; $\mathrm{n}=7, \mathrm{IL}-4^{-/-} \mathrm{T}$ cell injected; Student's t-test). (g) MyD88 $8^{-/-}$mice exhibit no difference in retinal ganglion cell number in the contralateral retinas compared to wild type mice. Retinal ganglion cell counts ( $\%$ of wild type) of wild type and MyD8 $88^{-/-}$mice were assessed by Fluoro-Gold staining ( $\mathrm{n}=3$, wild type; $\mathrm{n}=3$, MyD88 ${ }^{--}$; Student's t-test). (h) Mice receiving MyD88 ${ }^{1-}$ bone marrow exhibit no difference in retinal ganglion cell number in the contralateral retinas compared to those receiving wild type bone marrow. Retinal ganglion cell counts ( $\%$ of wild type $\Rightarrow$ wild type) of wild type $\Rightarrow$ wild type or MyD88 $8^{-/-} \Rightarrow$ wild type bone marrow chimeras. Bone marrow was allowed to engraft for 6 weeks before optic nerve injury, and retinal ganglion cell survival was assessed by Fluoro-Gold staining $(\mathrm{n}=3$,

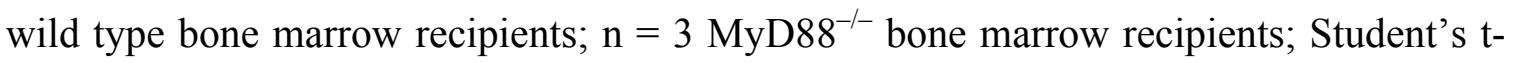
test). 

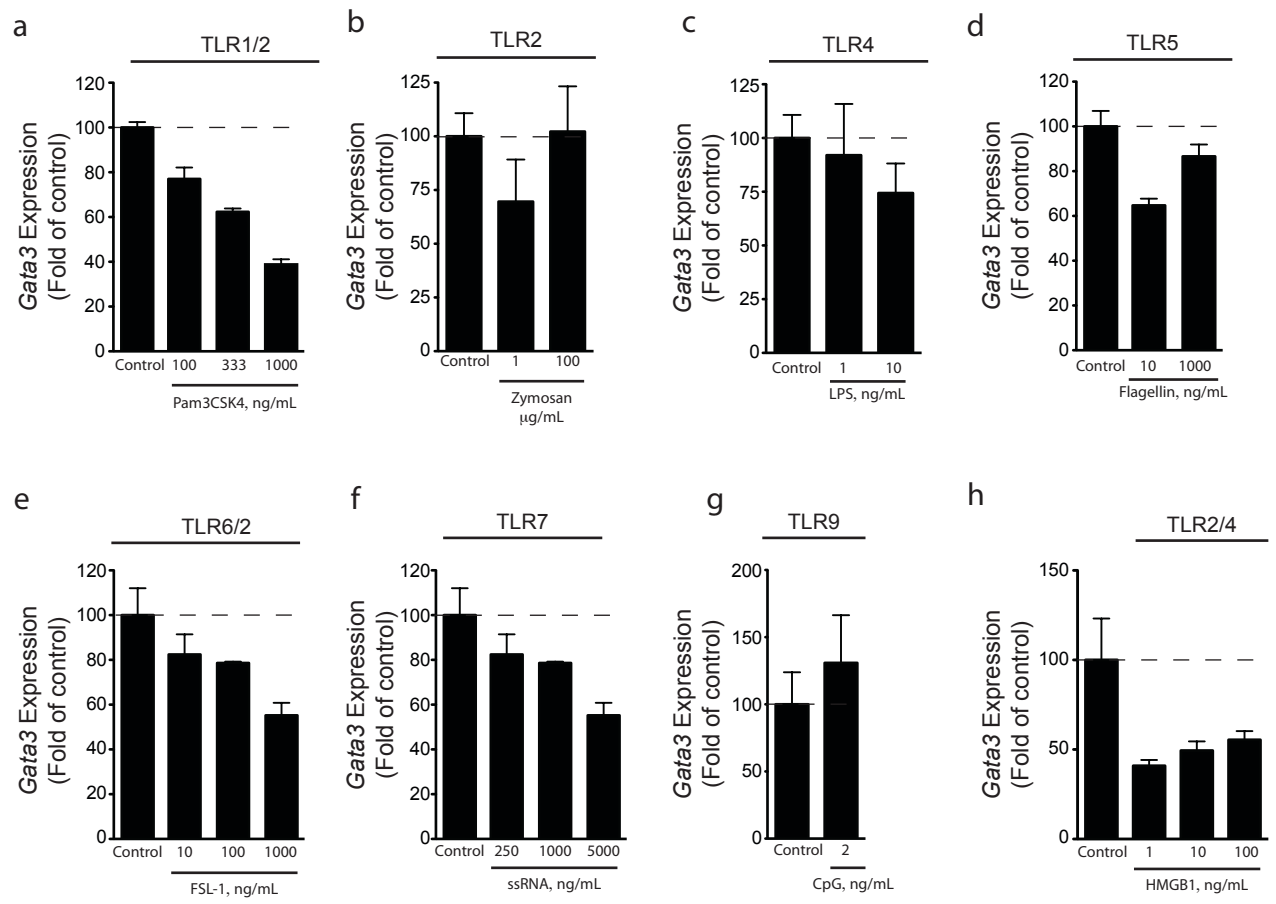

Supplementary Figure 3: Classical TLR ligands do not increase Gata3 mRNA expression in $C D 4^{+}$T cells. Gata3 expression (mean \pm s.e.m.) in sorted wild type $\mathrm{CD}^{+}$ T cells co-cultured with Pam3CSK4 (a), zymosan (b), LPS (c), flagellin (d), FSL-1 (e), ssRNA (f), CpG (g), or HMGB1 (h) at the indicated concentrations for 3 days ( $\mathrm{n}=3$ per group, representative of two experiments) 

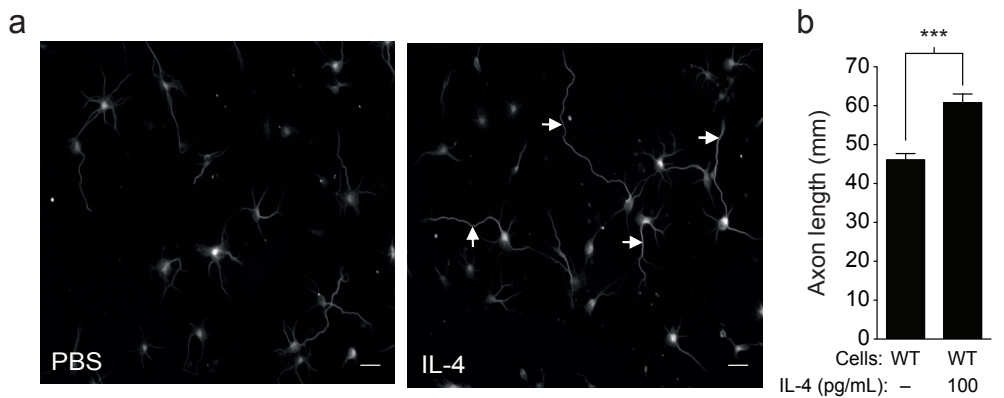

Supplementary Figure 4: IL-4 induces axon elongation in cultured neurons (a, b) The application of a single dose of $100 \mathrm{pg} / \mathrm{ml}$ recombinant IL-4 significantly increased the length of axons (mean \pm s.e.m.) of isolated primary neurons. Representative microphotographs (a) and quantification of axonal length (b) of isolated neurons treated with PBS or a single dose of $100 \mathrm{pg} / \mathrm{ml}$ recombinant IL-4. Scale bar: $20 \mu \mathrm{m}$. (n[well] $=4$ per group. ${ }^{* * *}, \mathrm{p}<0.001 ; *, \mathrm{p}<0.05$; One-way ANOVA with Bonferroni's post-test). 


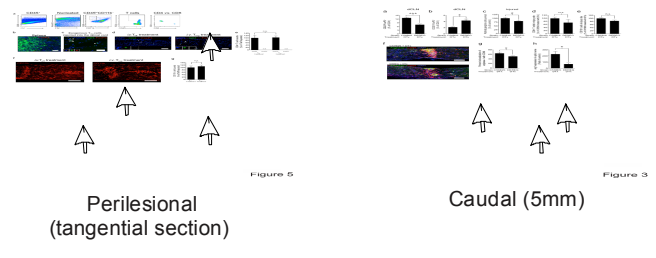

Supplementary Figure 5: T cells migrate caudally from the lesion site after injection. CFDA $_{\mathrm{SE}}$ labeled T cells were injected into the site of injury immediately after spinal cord injury. Spinal cords were isolated and visualized for $\mathrm{CFDA}_{\mathrm{SE}}$ labeled T cells 6 days after the lesion. (Scale bars: $15 \mu \mathrm{m}$ ). T cells were found at least $5 \mathrm{~mm}$ away from the site of injection, where axonal regrowth was observed. 
a
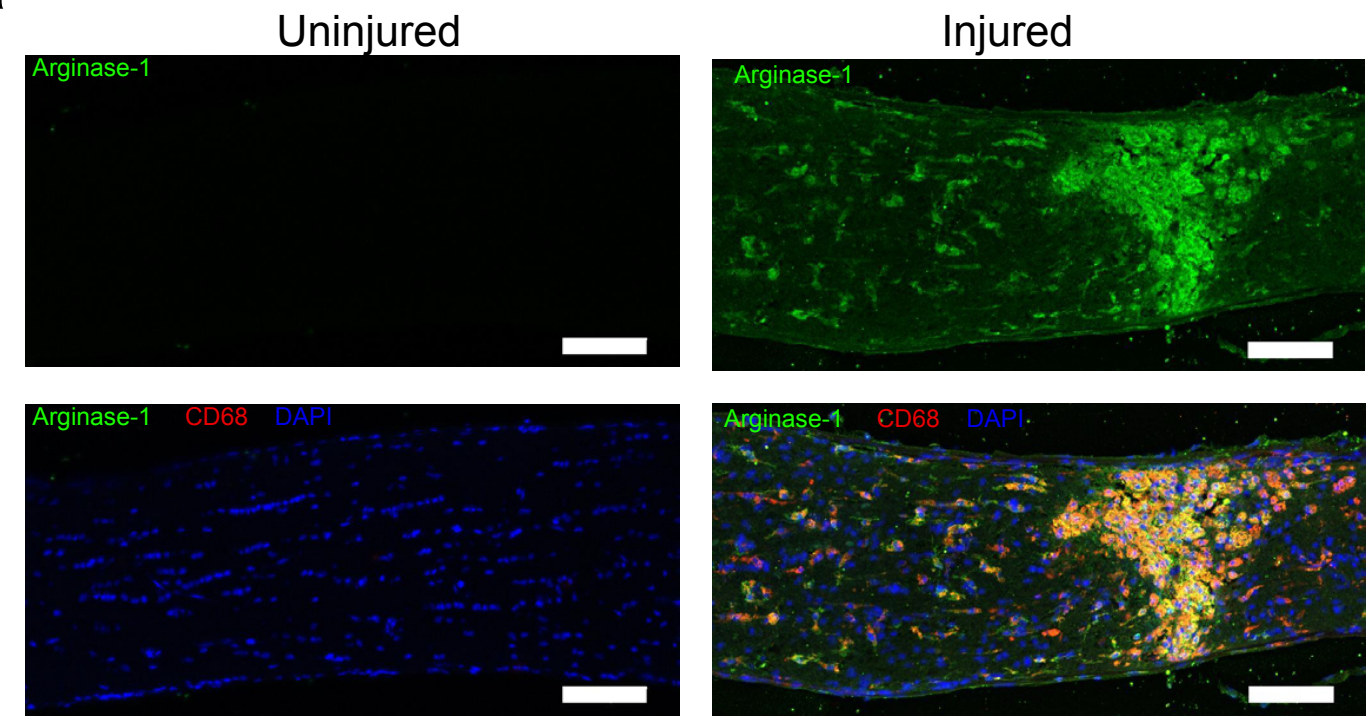

\section{Uninjured}

b

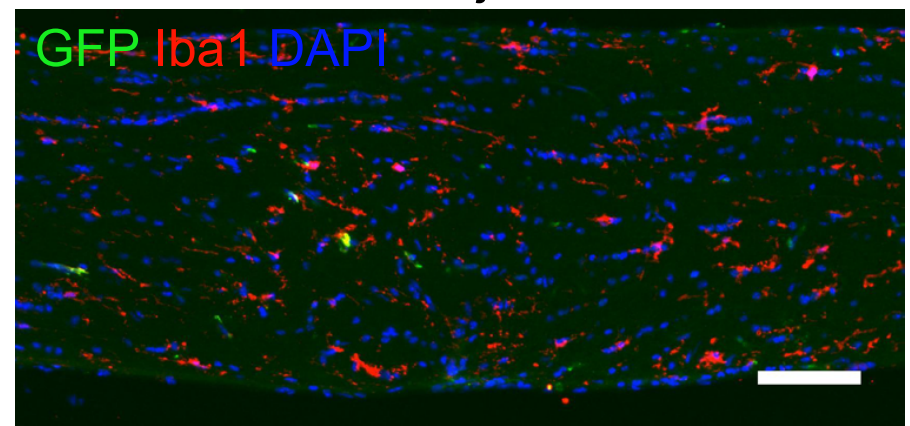

Supplementary Figure 6: Uninjured optic nerves have few $\mathrm{GFP}^{+} \mathrm{Ibal}^{+}$microglia and little arginase-1, but arginase-1 staining is greatly increased after injury. (a)

Immunohistochemical staining of arginase-1 (green) and CD68 (red) in the uninjured and 
injured optic nerve (scale bar $=100 \mu \mathrm{m}$ ). (b) Immunohistochemical staining of an optic nerve in a C57B1/6 mouse that was lethally irradiated and transplanted with GFP bone marrow showing sparse transplanted $\mathrm{GFP}^{+}$cells (green) among plentiful resident $\mathrm{Ibal}^{+}$ microglia (red). Image is from uninjured nerve 6 weeks post-transplantation (scale bar $=$ $100 \mu \mathrm{m})$. 
a

$T_{\text {reg }}$ in blood at time of injury

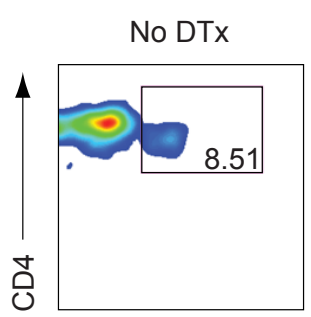

FoxP3
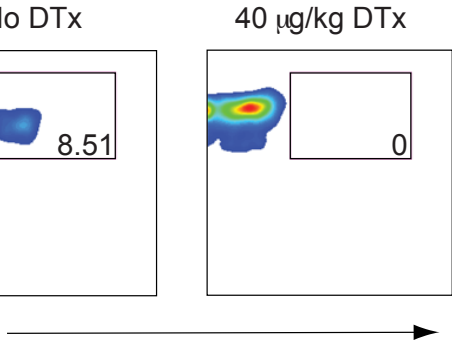

d
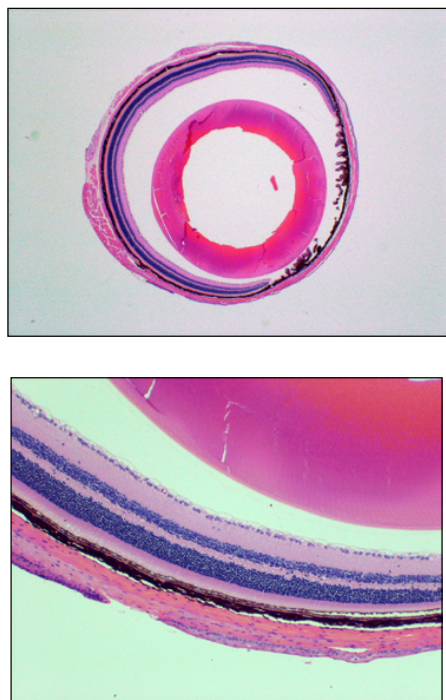

b

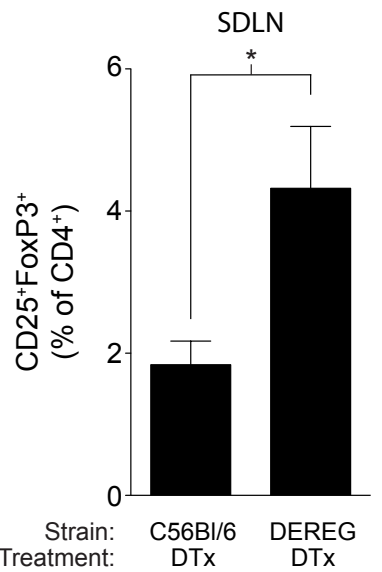

C Uninjured

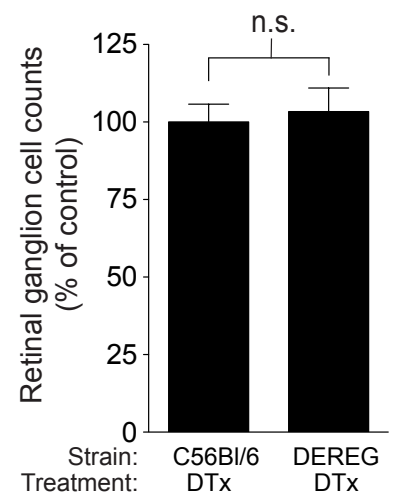

e
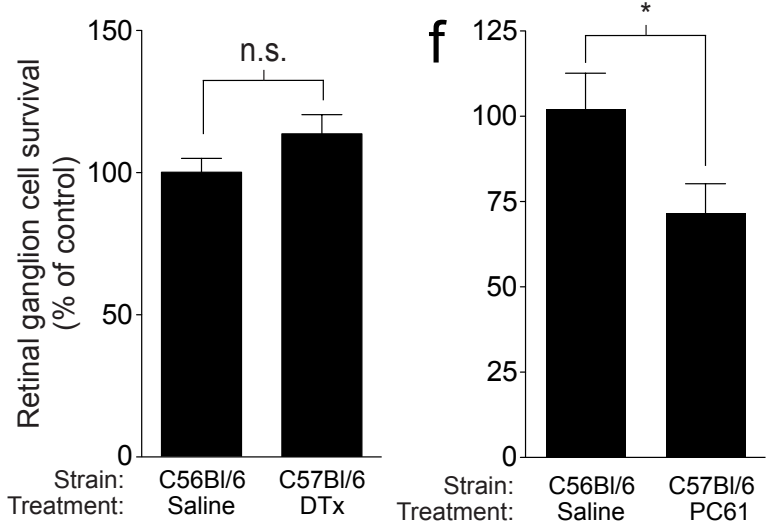

Supplementary Figure 7: Characterization of $40 \mu \mathrm{g} / \mathrm{kg}$ DTx. treatment. (a) Flow cytometry on the day of injury of CD4 and Foxp3 in peripheral blood of DEREG mice 
treated with vehicle or $40 \mu \mathrm{g} / \mathrm{kg}$ DTx. (b) Flow cytometry in the skin-draining lymph nodes of DEREG or wild type littermates treated with DTx two days before injury and on the day of injury, showing percent of $\mathrm{CD} 25^{+} \mathrm{Foxp}^{-} \mathrm{T}_{\text {eff }}$ cells, graphed as a percentage of TCR $\beta^{+} \mathrm{CD} 4^{+}$cells $(\mathrm{n}=12$ wild type and 9 DEREG treated mice; *, $\mathrm{p}<0.05$, Student's $\mathrm{t}-$ test; representative of three experiments). (c) Representative images of H\&E staining in eyes of DEREG mice treated with $40 \mu \mathrm{g} / \mathrm{kg}$ DTx. Abnormal architecture or immune cell infiltration was found in 0/17 C57Bl/6 and 0/21 B6AF1 mice. (d) Retinal ganglion cell counts from DEREG and wild type mice injected with $40 \mu \mathrm{g} / \mathrm{kg}$ DTx two days before injury and on the day of injury ( $\mathrm{n}=19$ wild type and 25 DEREG; Student's t-test; representative of three experiments). (e) No difference was observed in RGC survival of C57B1/6 mice treated with $40 \mu \mathrm{g} / \mathrm{kg}$ DTx or saline 2 days before injury and on the day of injury ( $n=10$ mice per group; Student's t-test representative of 2 experiments). 
a

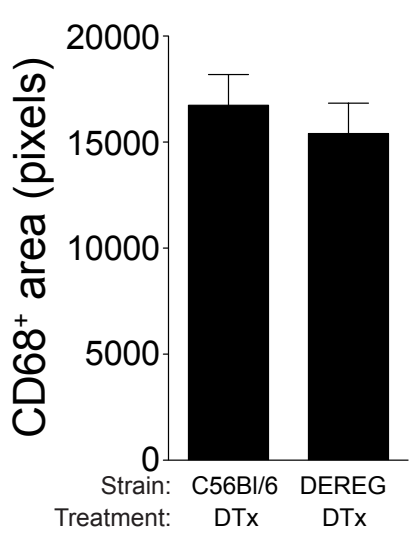

b

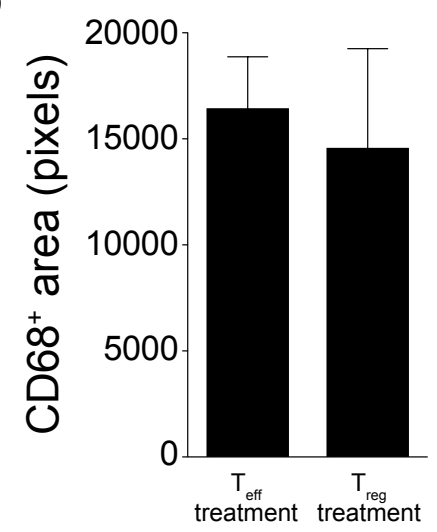

Supplementary Figure 8: There is no change in $C D 68^{+}$area after injury with $T_{\text {reg }}$ manipulation. (a) Quantification of $\mathrm{CD} 68^{+}$area in immunohistochemical staining of injured optic nerves of C57Bl/6 treated with DTx or DEREG mice treated with DTx ( $\mathrm{n}=$ 3 C57B1/6 treated with DTx and 9 DEREG treated with DTx Student's t-test;

representative of two experiments). (b) Quantification of $\mathrm{CD} 8^{+}$area in immunohistochemical staining of injured optic nerves of C57Bl/6 mice injected with $1 \times 10^{6} \mathrm{~T}_{\text {eff }}$ or $\mathrm{T}_{\text {reg }}$ cells 2 days before injury and on the day of injury $\left(\mathrm{n}=9 \mathrm{~T}_{\text {eff }}\right.$ injected and $6 \mathrm{~T}_{\text {reg }}$ injected; Student's t-test; representative of two experiments). 

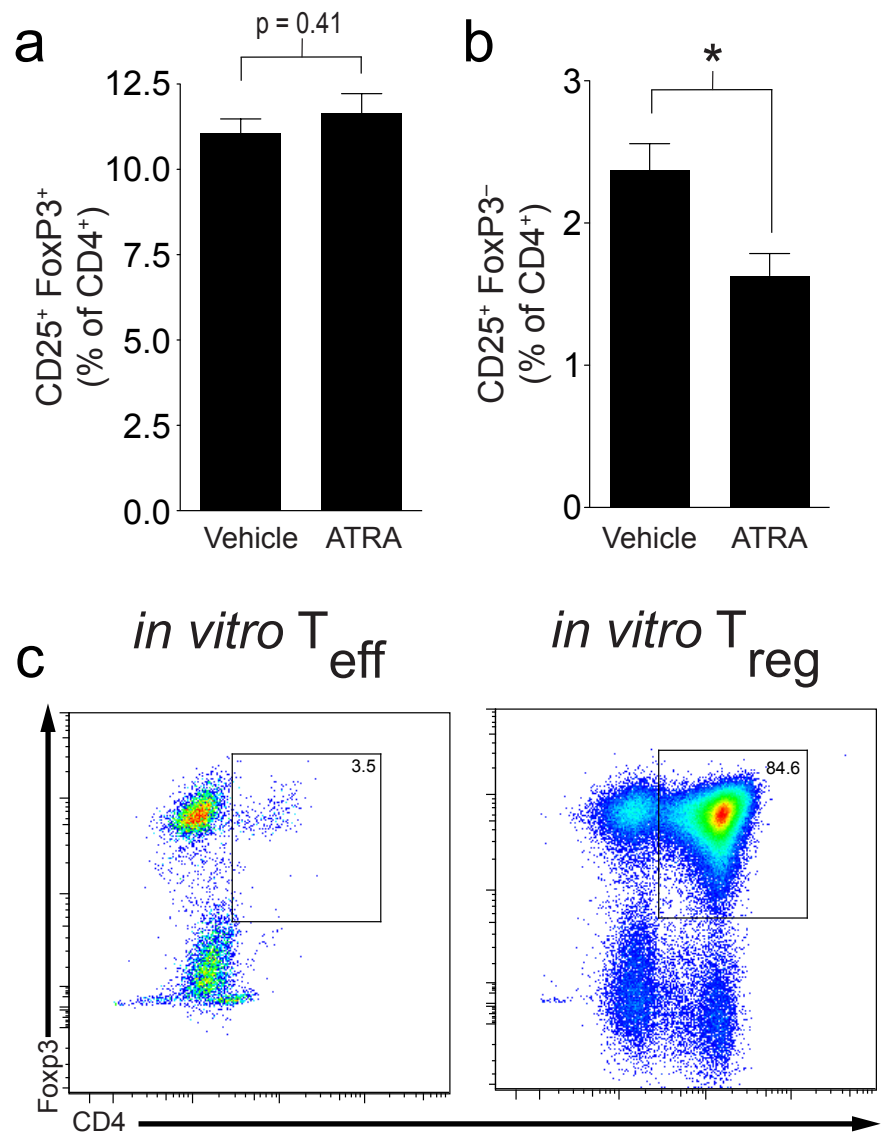

Supplementary Figure 9: ATRA treatment leads to decreased $T_{\text {eff }}$ proportion in the skindraining lymph nodes and to increased $T_{\text {reg }}$ proportion in vitro. (a, b) Flow cytometry in the skin-draining lymph nodes of wild type treated with vehicle or ATRA showing percent of CD25 $5^{+}$Foxp $^{+} \mathrm{T}_{\text {reg }}$ cells (a) and of CD $25^{+}$Foxp $^{-} \mathrm{T}_{\text {eff }}$ cells (b), graphed as a percentage of TCR $\beta^{+} \mathrm{CD}^{+}$cells $(\mathrm{n}=7$ vehicle treated and $\mathrm{n}=9$ ATRA treated; $*, \mathrm{p}<$ 0.05, Student's t-test; representative of two experiments). (c) Representative flow cytometry plots of viable cells in lymph node cultures that have been treated with $10 \mathrm{nM}$ ATRA, $5 \mathrm{ng} / \mathrm{mL}$ TGF $\beta, 250 \mathrm{U} / \mathrm{mL}$ IL-2, $1 \mu \mathrm{g} / \mathrm{mL}$ anti-CD3, and $1 \mu \mathrm{g} / \mathrm{mL}$ anti-CD28 ( $\mathrm{T}_{\text {reg }}$ ) or only $1 \mu \mathrm{g} / \mathrm{mL}$ anti-CD3, and $1 \mu \mathrm{g} / \mathrm{mL}$ anti-CD28 ( $\mathrm{T}_{\text {eff }}$ ) for 5 days. The boxes 
denote the $\mathrm{CD}^{+}{ }^{+}$Foxp $3^{+} \mathrm{T}_{\text {reg }}$ population, and numbers represent percent of viable cells that are $\mathrm{CD} 4^{+} \mathrm{Foxp}^{+}$. 